J Med Chem. 2017 October 12; 60(19): 8055-8070. doi:10.1021/acs.jmedchem.7b00780.

\title{
Design and Structure-Guided Development of Novel Inhibitors of the Xeroderma Pigmentosum Group A (XPA) Protein-DNA Interaction
}

\author{
Navnath S. Gavande ${ }^{\dagger,}$, , Pamela VanderVere-Carozza ${ }^{\dagger}$, Akaash K. Mishra $^{\dagger, \ddagger}$, Tyler L. \\ Vernon $^{\dagger}$, Katherine S. Pawelczak ${ }^{\S}$, John J. Turchi ${ }^{\dagger, \ddagger, \S,{ }^{*}}$ \\ † Department of Medicine, Indiana University School of Medicine, Indianapolis, Indiana 46202, \\ United States \\ ‡ Department of Biochemistry and Molecular Biology, Indiana University School of Medicine, \\ Indianapolis, Indiana 46202, United States \\ $\S$ NERx Biosciences, 212 W 10th Street, Suite A480, Indianapolis, Indiana 46202, United States
}

\begin{abstract}
XPA is a unique and essential protein required for the nucleotide excision DNA repair pathway and represents a therapeutic target in oncology. Herein, we are the first to develop novel inhibitors of the XPA-DNA interaction through structure-guided drug design efforts. Ester derivatives of the compounds 1 (X80), 22, and $\mathbf{2 4}$ displayed excellent inhibitory activity $\left(\mathrm{IC}_{50}\right.$ of $0.82 \pm 0.18 \mu \mathrm{M}$ and $1.3 \pm 0.22 \mu \mathrm{M}$, respectively) but poor solubility. We have synthesized novel amide derivatives that retain potency and have much improved solubility. Furthermore, compound $\mathbf{1}$ analogs exhibited good specificity for XPA over RPA (replication protein A), another DNA-binding protein that participates in the nucleotide excision repair (NER) pathway. Importantly, there were no significant interactions observed by the X80 class of compounds directly with DNA. Molecular docking studies revealed a mechanistic model for the interaction, and these studies could serve as the basis for continued analysis of structure-activity relationships and drug development efforts of this novel target.
\end{abstract}

\section{Graphical Abstract}

\footnotetext{
*Corresponding Authors N.S.G.: ngavande@iu.edu; phone, +1-317-248-6939, J.J.T.: jturchi@iu.edu; phone, +1-317-278-1996; fax, +1-(317)-274-0396.

Notes

The authors declare the following competing financial interest(s): Dr. Turchi is a co-founder and the Chief Scientific Officer of NERx Biosciences.

Supporting Information

The Supporting Information is available free of charge on the ACS Publications website at DOI: 10.1021/acs.jmedchem.7b00780. Molecular formula strings and biological data (CSV) Initial XPA inhibitory screening data, structures of commercially available compound 1 analogs, ${ }^{1} \mathrm{H}$ NMR spectra of synthesized final compounds, $E / Z$ isomers determination using NOESY NMR and molecular docking, overlays and docking poses of $Z$ and $E$ isomer of potent XPA inhibitors, and 2D interactions of XPA inhibitors with XPA protein (PDF)
} 


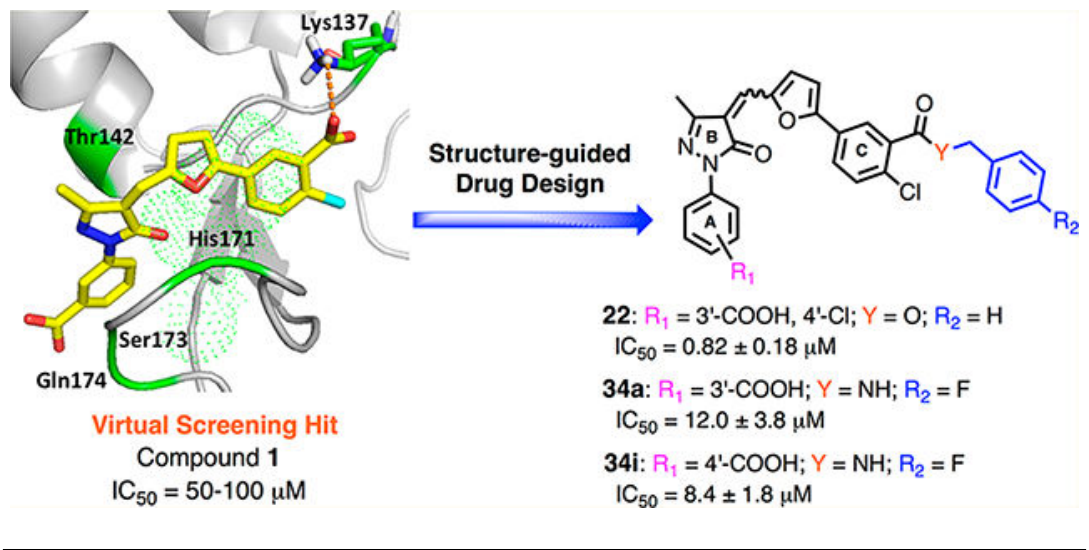

\section{INTRODUCTION}

Targeting DNA repair and the DNA damage response for cancer therapy has gained increasing attention with the recent U.S. FDA (December 2014) approval of the poly-ADP ribose polymerase (PARP) inhibitor olaparib (Lynparza, AstraZeneca) as the first DNA repair targeting agent for cancer treatment. ${ }^{1,2}$ While olaparib is approved as a single agent, the full utility of DNA repair targeted inhibitors can be expanded by their use in combination treatment regimens with DNA damaging chemotherapeutics including the platinum (Pt)based agents cisplatin, carboplatin, and oxaliplatin. ${ }^{3}$ However, this utility requires knowledge of the relevant repair pathways involved in repairing and tolerating platinuminduced DNA damage.

Approximately half of all cancer patients who receive anticancer chemotherapy are treated with a platinum drug at some point within their treatment regimen with widely varied outcomes. Most cancers display a good initial response, but unfortunately treatment failure ensues due to development of intrinsic or extrinsic drug resistance. ${ }^{4}$ There are multiple factors involved in platinum resistance; among them increased capacity of DNA damage repair is one of the major concerns. ${ }^{5}$ Therefore, we have focused on directly targeting the nucleotide excision repair (NER) pathway which is responsible for repairing the vast majority of platinum-induced DNA damage. ${ }^{1,6-8}$ Several studies revealed that the overexpression of NER proteins (mainly XPA, RPA, ERCC1, XPC, and XPF) and repair of DNA damage by these proteins are directly linked to platinum resistance which ultimately hampers the efficacy of platinum-based therapy. ${ }^{6,9-14}$ The suppression of NER activity has been potentially recognized as a highly effective adjuvant therapy with DNA damaging agents such as platinum drugs and radiotherapy toward maximizing efficacy, overcoming resistance, and reducing the toxicities associated with the current regimen. The DNA damage recognition process is the limiting step in NER pathway, and specifically the XPA (xeroderma pigmentosum group A) protein binds to the damaged duplex DNA in the DNA damage identification and verification process. ${ }^{15,16}$ XPA does not possess any enzymatic activity but is an integral component for which there is no redundant or compensatory protein. ${ }^{17,18}$ In addition, XPA has been shown to have a greater affinity for damaged DNA over undamaged DNA and is required for the removal of all types of DNA lesions repaired by NER. In fact, as there are no redundant proteins that can compensate for the loss of XPA 
activity, decreased expression of XPA has been observed in testicular cancers where 95\% of patients are cured by a platinum-based therapy. ${ }^{16,19,20}$ Therefore, XPA is a novel therapeutic target and the chemical synthetic lethality approach can be exploited with XPA inhibitors to increase the effectiveness of platinum-based chemotherapy in cancers in which there is only modest clinical success (Figure 1). ${ }^{6}$ While NER is responsible for removal of Pt-DNA damage, the homologous recombination repair (HRR) pathway allows the tolerance of PtDNA damage. We have devised a chemical synthetic lethality approach that provides novel mechanisms to specifically target cancer cells while sparing normal noncancer cells and thereby reducing toxicity associated with treatment (Figure 1). This model is fundamentally different from that of the synthetic lethality described for PARP inhibitors, which relies on endogenous single strand DNA damage to be converted to DNA double strand breaks, ${ }^{21-23}$ while our approach is designed specifically for Pt-combination therapy.

Human XPA is a relatively small 273 -residue protein $(39 \mathrm{kDa})$ that contains multiple domains and interaction motifs that support binding to DNA and other DNA repair proteins (Figure 2). ${ }^{17,18,24,25}$ The recently refined structural analysis of human XPA revealed that DNA binding activity resides in a 142 amino acid (XPA98-239) minimal DNA binding domain (MBD/DBD) spanning from the $\mathrm{C} 4$ zinc finger through the a-helix basic motif. ${ }^{26,27}$ The zinc containing globular core of XPA is responsible for binding both the ssDNA to dsDNA junction (Y junction) and the RPA70 domain. ${ }^{28,29}$ More recently, Koch et al. reported the first high-resolution X-ray crystal structures of the MBD of the yeast XPA homolog Rad14 bound to damage containing duplex DNA with either a cisplatin lesion (1,2GG) or an acetylaminofluorene adduct (AAF-dG). ${ }^{30}$ The interaction of XPA homolog Rad14 with the ss-dsDNA junction is consistent with previous studies indicating that human XPA also preferentially binds to the DNA junction. ${ }^{31}$ Inhibitors targeting XPA-DNA interfaces hold great potential to enhance the efficiency of treatment with DNA damaging agents and reverse the platinum drug resistance by reducing NER activity. Surprisingly, despite the potential physiological significance and extensive scientific progress on XPA protein, very little progress has been made to date to develop small molecule inhibitors targeting XPA. In our previous studies, ${ }^{8}$ a 3 D structure of the XPA MBD revealed a cleft that includes a number of conserved basic amino acids which has direct contact with the DNA in conjunction with surrounding residues and it also has an impact on binding to kinked DNA substrates, presumably similar to those formed by bulky DNA adducts that are repaired by NER. ${ }^{32,33}$ With further structure-based in silico screening of a virtual small molecule library targeting this cleft, we identified 5-(5-((1-(3-carboxyphenyl)-3-methyl-5-oxo-1,5dihydro-4H-pyrazol-4-ylidene)methyl)furan-2-yl)-2-chlorobenzoic acid 1 (X80 or TDRL$\mathrm{X} 80$, Figure $3 \mathrm{~A})^{8}$ as an XPA-DNA interaction inhibitor by using fluorescence polarization and ELISA assays. In this continuation of our research, we are the first to report the structure-guided development of the X80 class of XPA small molecule inhibitors with the identification of more potent inhibitors of the XPA-DNA interaction. 


\section{Results and Discussion}

\section{Initial Screening of XPA-DNA Interaction Inhibitors}

By use of the 3D structure of XPA determined by solution NMR (PDB code 1XPA), the coordinates of the C-terminal subdomain (residues 131-210) were found to have direct contact with a DNA ligand. Therefore, during our previous studies ${ }^{8}$ and also in these studies, we have targeted the cleft consisting of amino acid residues 138-142, 165-171, 174, and 177-181 for small molecule docking. Initial molecular docking studies with the compound 1 revealed that the interaction of compound $\mathbf{1}$ carboxylic acid (ring $\mathrm{C}$ ) with the cleft contacting Lys137 is critical for inhibitory activity, and there is a large space-filling pocket around aromatic ring $\mathrm{C}$ that can be exploited for further structural optimization (Figure 3B). To further investigate the feasibility of targeting druglike binding pockets and identify inhibitors with improved potency, we first searched the virtual ChemDiv (San Diego, CA, USA) and AKos GmbH (Steinen, Germany) library for compound 1 analogs with a criterion of 8595\% structural similarity. Approximately 30 commercially available analogs (Table 1) were acquired and tested for their activity in inhibiting the XPA-DNA interaction. Purified full length XPA protein was used to screen a series of compound $\mathbf{1}$ analogs in an electrophoretic mobility shift assay (EMSA) $29,34,35$ that involved binding to a duplex DNA substrate containing a site specific cisplatin-DNA adduct centrally located on the duplex. In this study, we have expanded the in vitro assays to include EMSA to avoid the spectrophotometric interference by the colored nature of the XPA compounds. This allowed us to validate our findings and further refine the structure-activity relationships necessary for XPA inhibition. The results presented in Figure 4 (see Supporting Information Figure S1 for \% DNA binding data) reveal a wide array of activities ranging from no inhibition to $100 \%$ inhibition at the relatively high concentration of $50 \mu \mathrm{M}$ tested. Compounds that displayed greater than $80 \%$ inhibition at $50 \mu \mathrm{M}$ were analyzed in titration experiments to determine $\mathrm{IC}_{50}$ values (Table $1)$.

\section{Optimization of Ring C and SAR Analysis}

Quantification of the EMSA data and additional concentration dependent analysis of compound $\mathbf{1}$ analogs provided potential insight into the structure-activity relationships. The $\mathrm{IC}_{50}$ values, reported in Table 1, showed that the most efficient inhibitors of XPA-DNA binding were compounds $19\left(\mathrm{IC}_{50}=1.6 \pm 0.8 \mu \mathrm{M}\right), \mathbf{2 2}\left(\mathrm{IC}_{50}=0.82 \pm 0.18 \mu \mathrm{M}\right), \mathbf{2 3}\left(\mathrm{IC}_{50}=\right.$ $1.93 \pm 0.86 \mu \mathrm{M})$, and $24\left(\mathrm{IC}_{50}=1.3 \pm 0.22 \mu \mathrm{M}\right)$. In agreement with the postulated molecular docking binding mode, the in vitro data indicate that the improved inhibitory activity of the compound $\mathbf{1}$ analogs is strongly related to the increasing hydrophobic interactions (by masking ionic carboxylic acid at ring $\mathrm{C}$ ) in the binding pocket of XPA. Nitro and halogen substitutions on ring $\mathrm{C}$ (compounds $\mathbf{5}$ and $\mathbf{6}$ ) exhibited a considerable increase in XPA inhibitory activity in comparison with carboxylic acid substitution on ring $\mathrm{C}$ (compounds $\mathbf{1}$ 4). The replacement of $2^{\prime}$ - $\mathrm{Br}$ substitution (6) with $2^{\prime}$-OMe (7) on ring $\mathrm{C}$ in nitro containing compound retained XPA inhibitory activity. However, the inclusion of $2^{\prime}-\mathrm{OH}(\mathbf{8})$ and $2^{\prime}$ $\mathrm{CH}_{3}(\mathbf{9})$ at position 2 on ring $\mathrm{C}$ resulted in a decrease in inhibitory activity.

A decreased inhibitory activity observed for $3^{\prime}$-substituted acetyl containing compound $\mathbf{1 0}$ as compared to ester derivative (11) shows a clear agreement with our initial docking studies 
that carboxylic acid or ester substitution on ring $\mathrm{C}$ is very important to maintain the interaction with Lys137 and to retain inhibitory activity.

A series of $3^{\prime}$-substituted esters (compounds 11-24) resulted in increased inhibitory activity. A trend in inhibition was observed in the analysis of methyl, ethyl, and propyl esters where the addition of each methyl group resulted in a $10 \%$ increase in XPA inhibitory activity. There was an increase in the activity when replacing a bioisosteric methyl group (compound 11) with a trifluoromethyl group (compound 12) on the pyrazolone ring (ring B). An addition of a chloro group on ring A exhibited a slight increase in the XPA inhibitory activity (compound $\mathbf{2 4}$ vs compound $\mathbf{2 3}$ ).

All binding analyses thus far have employed using the full length human XPA; however while doing these studies, we have also performed binding studies with the minimal DNAbinding domain (DBD) consisting of amino acids 98-239 (XPA98-239). To ensure the inhibitor effects of the compounds via binding to this domain of XPA, we have overexpressed and purified the XPA DBD as an N-terminal tagged construct. We have evaluated the activity of compound $\mathbf{1}$ and one of our most potent inhibitor, 22, on purified minimal DNA-binding region XPA98-239 using EMSA. Figure 5 shows inhibition of full length XPA and minimal DBD XPA98-239 with increasing concentrations of compound 1 and compound 22. Interestingly, the compound $\mathbf{1}$ displayed greater potency toward full length XPA compared to the DNA-binding region $\mathrm{XPA}_{98-239}$, and this difference was dramatically reduced when assessing compound $\mathbf{2 2}$. These data suggest that there may be other interactions beyond the minimal DBD that stabilize the interactions of compound $\mathbf{1}$ with XPA but are not required for the higher affinity of compound 22.

Compounds 19, 22, 23, and 24 displayed excellent inhibitory activity $\left(\mathrm{IC}_{50}=1.6 \pm 0.8 \mu \mathrm{M}\right.$, $0.82 \pm 0.18 \mu \mathrm{M}, 1.93 \pm 0.86 \mu \mathrm{M}$, and $1.3 \pm 0.22 \mu \mathrm{M}$, respectively); however each of the commercially available compounds showed poor solubility. Therefore, with the aim to improve solubility, to establish a structure-activity relationship, and to define the structural determinants required to inhibit the XPA-DNA interactions, we synthesized and evaluated several novel compound $\mathbf{1}$ analogs.

\section{Chemistry}

We have developed a six- to seven-step synthesis for novel XPA inhibitors with an overall yield of $\sim 15-20 \%$ using a highly efficient and operationally convenient protocol. The synthesis of final target compounds 34a-k is depicted in Scheme 1. We have obtained precursors 31a,b (Scheme 1) or 37a,b (Scheme 2) from starting materials 25a/25b by modifying existing synthetic approaches. ${ }^{36-39}$ Generally, the substituted dihydro- $1 \mathrm{H}$ pyrazol-1-yl 26a,b were prepared by refluxing corresponding substituted phenylhydrazines $\mathbf{2 5 a}, \mathbf{b}$ with ethyl acetoacetate in acetic acid. The esterification of carboxylic acids $\mathbf{2 6 a}, \mathbf{b}$ using $\mathrm{H}_{2} \mathrm{SO}_{4}$ in ethanol furnished 27a,b in good yields. Aldehyde $\mathbf{3 0}$ was prepared in high yield by optimizing Pd-catalyzed Suzuki coupling of 5-bromofuran-2-carbaldehyde $\mathbf{2 8}$ with boronic acid 29. Precursors 31a,b were prepared in moderate to good yields as a mixture of $E / Z$ isomers by condensing corresponding pyrazolones $\mathbf{2 7} \mathbf{a}, \mathbf{b}$ with aldehyde $\mathbf{3 0}$ in acetic acid under Knoevenagel reaction condition. To confirm the activity of compound $\mathbf{1}$, we have 
synthesized compound $\mathbf{1}$ and its $4^{\prime}$-substituted analog 32 by hydrolysis of corresponding esters (31a and 31b) using $2 \mathrm{~N} \mathrm{NaOH}$ at room temperature. Alkyl- and arylamides 33a-j were prepared from 31a/31b in moderate yields by utilizing EDCI/HOBt amide synthetic protocol. Final target compounds $34 \mathbf{a}-\mathbf{d}$ and $\mathbf{3 4 f}-\mathbf{k}$ were obtained from compounds $33 \mathbf{a}-\mathbf{j}$ by hydrolysis of corresponding esters using $10 \mathrm{~N} \mathrm{LiOH}$ at room temperature. Target compound 34e was obtained from 34d and cyclopropylmethylamine using EDCI/HOBt amide synthesis strategy.

To explore the effects of amide substitution on para-position at ring $\mathrm{C}$, we have synthesized target compounds 39a,b as depicted in Scheme 2 Aldehyde 36 was prepared from 5bromofuran-2-carbaldehyde $\mathbf{2 8}$ and boronic acid $\mathbf{3 5}$ using in house optimized Suzuki coupling reaction by an above-described synthesis of aldehyde 30. Precursors $\mathbf{3 7 a}, \mathbf{b}$ were prepared in moderate to good yields by condensing corresponding pyrazolones $\mathbf{2 7 a}, \mathbf{b}$ with aldehyde 36 by using an above-described Knoevenagel reaction condition. Final compounds 39a-d were prepared from 37a or 37b using EDCI/HOBt amide synthesis protocol, followed by ester hydrolysis of compounds 38a-d. All biologically important compounds are $\mathbf{2 5 \%}$ pure as determined by HPLC coupled to electrospray ionization mass spectrometry (LC/ESI-MS) analysis. Importantly, this protocol is feasible for large scale production, and synthesis on a $2 \mathrm{~g}$ scale is easily achievable. Esters $\mathbf{3 3} \mathbf{a}-\mathbf{j}$ and final compounds 34a-k, 39a-d exist as a mixture of $E / Z$ isomers, whereas $Z$ isomer is the major product $(\sim 70-80 \%)$ as determined by NOESY NMR analysis of a mixture of isomers (see the Supporting Information Figure S2 for details). While we performed separation of the $E / Z$ isomers of our final compounds using preparative HPLC, each isolated isomer rapidly equilibrated to give back the original mixture of isomers, and therefore separation of two isomers is practically impossible.

\section{Structural Modification of Compound 22 To Improve Solubility and Further SAR Analysis}

The identification of compounds $\mathbf{2 2}$ and $\mathbf{2 4}$ as the most potent XPA inhibitors in the ester series limited by poor solubility led us to focus our synthetic efforts on improving the aqueous solubility profile. The ester-containing compounds exhibited a high cLogP, and therefore, we utilized small isosteric modifications to improve solubility and metabolic stability. This was accomplished by replacing the metabolically labile ester group with a more chemically and metabolically stable amide. The novel synthesized compounds' in vitro data are presented in Table 2. Replacement of the ester in compound $\mathbf{2 2}\left(\mathrm{IC}_{50}=0.82 \pm 0.18\right.$ $\mu \mathrm{M})$ with an amide and addition of a $p$-fluoro group $34 \mathbf{a}\left(\mathrm{IC}_{50}=12 \pm 3.8 \mu \mathrm{M}\right)$ led to dramatically increased solubility and decreased in potency but at least retained low micromolar activity. The difference in activity might be due to their ability to interact with a critical amino acid, Lys137, to retain and improve XPA inhibitory activity or might be due to result of the balance between the differences in the strength of interactions with another important cleft containing XPA backbone amino acid residues.

Subsequently, several alkylamide- and arylamide-containing substituents at ring $\mathrm{C}$ were investigated as represented by $\mathbf{3 4 b} \mathbf{b} \mathbf{h}$, with 4-fluorobenzyl (34a), dimethoxyphenyl (34c), and cyclopropylmethyl (34d) being the optimal fit in the space filling pocket. Cyclopropylmethyl containing amide $\mathbf{3 4 d}\left(\mathrm{IC}_{50}=9.8 \pm 2.6 \mu \mathrm{M}\right)$ at ring $\mathrm{C}$ exhibited almost 
3.2-fold increased in activity compared to cyclopropylamide $34 \mathbf{f}\left(\mathrm{IC}_{50} \approx 32 \mu \mathrm{M}\right)$, aligning with our previous inhibition trend observed for extended ester derivatives (13-21). Insertion of a heteroatom such as oxygen into the cyclohexane ring in the form of a pyran was tolerated, with more preference for the extended 4-methyltetrahydropyran $34 \mathrm{~h}\left(\mathrm{IC}_{50}=14.3\right.$ $\pm 2.1 \mu \mathrm{M})$ over the 4-tetrahydropyran $34 \mathrm{~g}\left(\mathrm{IC}_{50} \approx 40 \mu \mathrm{M}\right)$. The amide substitution at 4position of ring $\mathrm{C}$ with fluorobenzyl (39a) and cyclopropylmethyl (39b) showed no XPA inhibitory activity ( $\mathrm{IC}_{50} \geq 50 \mu \mathrm{M}$ ), which may be due to structural constraints imposed by such a modification to fit in the space-filling binding cleft. On the contrary, 4-substitued fluorobenzylamide (39c) and cyclopropylmethylamide (39d) at ring $\mathrm{C}$ along with parasubstituted carboxylic acid on ring A showed potent XPA inhibitory activity in EMSA. Most likely, structural conformations of compounds $39 \mathrm{c}$ and 39d allow flexibility in accommodating these inhibitors within the XPA binding cavity.

Simultaneously, we have evaluated the influence of the carboxylic acid (ring A) of the arylpyrazolone moiety by replacing it with ester and amide groups. EMSA analysis revealed a dramatically decreased or almost no XPA inhibitory activity for ester (33a) and amide (34e) containing compounds, highlighting the importance of the carboxylic acid at ring A to maintain the interaction with XPA backbone residues and ultimately to retain inhibition activity. Interestingly, 4-substitued carboxylic acid at ring A exhibited promising activity with 4-fluorobenzyl 34i $\left(\mathrm{IC}_{50}=8.4 \pm 1.8 \mu \mathrm{M}\right)$ and cyclopropylmethyl 34k $\left(\mathrm{IC}_{50}=9.1 \pm 2.4\right.$ $\mu \mathrm{M})$ amide containing compounds.

Overall, on the basis of XPA inhibition data (Table 1 and 2) confirmed by EMSA analysis, among the compounds those with the alkyl/alkylaryl ester substitution at ring $\mathrm{C}$ were the most potent XPA inhibitors. In addition, alkyl/alkylaryl amide substitution at ring $\mathrm{C}$ along with carboxylic acid substitution on 3-position or 4-position at ring A displayed inhibition of XPA-DNA interaction at submicromolar concentrations. These results strongly correlate with the molecular docking data (Figure 6), showing compounds 22 and 34i interact strongly by positioning themselves in the space-filling pocket and cleft of the XPA binding site.

\section{Molecular Docking}

Initial molecular docking studies allowed us to expand our SAR and potentially correlate the results from the XPA inhibitory studies with the docking-based binding analysis of our compounds. To delineate the key interactions responsible for differences in binding affinity and to understand the SAR, the structures of XPA inhibitors were flexibly docked into the XPA minimal DNA binding domain (PDB code 1XPA). Docking studies with the most active XPA inhibitors revealed that only the $Z$ isomer can be effectively docked in the XPA binding cavity. Binding modes for the $Z$ isoform is predictable and consistent, while the $E$ isoform does not adopt a consistent binding. This is likely an indication that $E$-isomer is inactive (see the molecular docking section in the Supporting Information for more details). Compounds with methyl substitution on pyrazolone ring have a higher ratio of the $Z$-isomer $(\sim 70-80 \%)$ as determined by NOE NMR analysis. As docking studies predicted, only the $Z$ isomer is likely contributing to the XPA inhibition activity; therefore we expect lower $\mathrm{IC}_{50}$ values (greater potency) with the compounds displayed in Tables 1 and 2. All $Z$-isomer compounds bind similarly to parent compound $\mathbf{1}$ by extending through the cleft and 
positioning ring $\mathrm{C}$ ester/amide in range for interactions with a critical amino acid, Lys137. As with the ester (11-24) and amide (34a-k and 39a-d) derivatives, alkyl or more bulky alkylaryl substituents at position 3 of aromatic ring $\mathrm{C}$ occupy the space filling binding pocket of the XPA binding site very efficiently.

Figure 6 shows the binding and molecular interactions of 22 and 34i $Z$-isomers within XPA MBD. The molecular interaction of 22 (Figure 6A) and 34i (Figure 6B) $Z$-isomer is largely ascribed to various electrostatic interactions, including the following. (i) Compound 22 ester carbonyl and compound 34i amide carbonyl make hydrogen bond contacts with the amine of Lys137. (ii) 3'-COOH (ring A) of compound 22 shows hydrogen bond contacts with the backbone amine of Gln174, while most interestingly, $4^{\prime}-\mathrm{COOH}$ of compound 34i does not interact with Gln174 but makes salt-bridge interactions with one of the most important cleft amino acid, Lys179 (Lys179 shown to impact binding to kinked DNA substrates). ${ }^{8}$ (iii) There are $\pi-\pi$ stacking interactions between the furan moiety and the aromatic ring of His171 in both compounds. In addition, compound 22 phenyl moiety is also well positioned to make additional $\pi-\pi$ stacking interactions with His171. (iv) Both substituted phenyl groups of ester (22) and amide (34i) are optimally positioned to make cation- $\boldsymbol{\pi}$ interactions with the cleft amino acid, Lys167. (v) Both compound's N1-N2 of pyrazolone ring position reveal the potential for hydrogen bond contacts with the hydroxyl group of Ser173 and also with the cleft amino acid, Thr142. Docking studies also predicted a stronger affinity of the ester containing $Z$-isomer of compound 22 than amide containing $Z$-isomer of compounds 34a, 34i, and 39c (see Figures S3-S6 in the Supporting Information for details). This fact could be a result of the balance between the differences in the strength of the $\pi-\pi$ stacking interactions between the furan as well as phenyl moieties and the aromatic ring of His171. Additionally, the increased and tighter interactions by the ester series of compounds with the XPA backbone amino acid residues Lys137, Gln174, and His171 might be one of the reasons that the ester containing compounds show higher affinities than the amide series of compounds. Particularly, the higher affinity of ester containing compound 22 than bioisoteric amide containing 34a might be due to it is potential to make hydrogen bond contacts with the backbone amine of the Gln174 while compound 34a lacks these interactions (see the Supporting Information Figures S3, S4, and S5 for details). On a particular note, these molecular docking studies represent models for potential interactions between our small molecule inhibitors (SMIs) and the XPA MBD region. The differences in inhibitory activity of our SMIs might be due to different structural conformation and their ability to interact with amino acid residues located within the XPA binding cleft.

\section{DNA Intercalation Assay and Specificity}

A competitive DNA intercalation assay was performed to determine if the compounds' activity could be the result of binding to DNA. To access this as a potential mechanism of inhibition, compounds $22, \mathbf{2 4}, \mathbf{3 4 a}, 34 \mathbf{3}, 3 \mathbf{3 4}, \mathbf{3 4 k}$, and $39 \mathrm{c}$ were analyzed in the fluorescence displacement assay along with using doxorubicin (Dox), a known noncovalent DNA binding chemotherapeutic, as a positive control. The results presented in Figure 7 demonstrate that no significant DNA binding activity was observed for recently synthesized novel XPA inhibitors (34a, 34d, 34i, 34k, and 39c). However, commercially available compounds 22 and 24 exhibited DNA binding activity above their $\mathrm{IC}_{50}$ value concentrations. These results 
are consistent with our XPA compounds that specifically inhibit protein-DNA interaction by binding to the XPA protein and not via binding to the DNA.

Furthermore, to access specificity of our compounds, we have evaluated inhibitory activity against RPA (replication protein A) by using our previously described EMSA. ${ }^{7}$ RPA is a single-stranded DNA binding protein and forms a complex with XPA in duplex damaged DNA binding activity (DNA damage recognition process) in the NER pathway. ${ }^{17,18,40,41}$ RPA is also involved in other metabolic pathways, including DNA replication and recombination. Most importantly, there was no RPA inhibition observed at $10 \mu \mathrm{M}$ with compounds 22, 24, 34i, and 34k in RPA-based EMSA assay.

\section{Conclusions}

Inhibition of macromolecular protein-DNA interactions by small molecules represents a relatively new class of important interactions that can be targeted for therapeutic intervention. Because of XPA's interaction with Pt-damaged DNA, its absolute requirement in the initiation of Pt-DNA-damage repair and with refined structural analysis of the protein, XPA has emerged as an attractive drug target for combination therapy. A broad range of cancers such as lung, ovarian, bladder, and head and neck will receive a Pt therapy with modest efficacy. Effective inhibition of XPA and ultimately preventing repair of Pt-DNA damage has the potential to sensitize these cancers and reverse resistance to afford better clinical outcomes.

Therefore, on the basis of our previous molecular insights, we have designed and identified highly potent inhibitors of the XPA-DNA interaction. Compounds 19, 22, 23, and 24 exhibited inhibition of XPA-DNA interaction at submicromolar concentration; however, further analysis of ester derivatives was hampered due to poor solubility. Further systematic structure-guided drug design and synthetic SAR analysis of compound $\mathbf{1}$ derivatives led to the identification of $\mathbf{3 4 a}, \mathbf{3 4}, \mathbf{3 4 d}, \mathbf{3 4 i}, \mathbf{3 4 k}$, and $39 \mathbf{c}$ as the most promising compounds in the series.

To our current knowledge, we are the first to develop highly potent and novel XPA inhibitors, and most importantly these compounds do not bind directly with DNA. In addition, these compounds do not interact with multifunctional RPA, a protein that forms a complex with XPA in duplex damaged DNA binding activity. These results offer new knowledge regarding the structural interaction of XPA inhibitors and its binding site that could be utilized in the design of future XPA inhibitors. Together the properties of these novel XPA inhibitors make them promising lead compounds for development into anticancer drugs that are to be used in combination therapy or as reagents for biochemical, cell and cancer biology studies. Further SAR analysis and biological evaluation of lead compounds are underway. 


\section{Experimental Details}

\section{General}

All commercially available compounds 1-24 were purchased from ChemDiv (San Diego, CA, USA) and AKos GmbH (Steinen, Germany) library with highest purity ( $₫ 95 \%$ ) and prepared at $10 \mathrm{mM}$ stock solution in $100 \%$ DMSO. All chemicals used for synthesis were purchased from Aldrich, Alfa Aesar, Acros, Fisher Scientific, AK Scientific, and CombiBlocks Chemical Co. (USA) and used without further purification. Anhydrous solvents were obtained from Fisher Scientific or Aldrich and used directly. All reactions involving air- or moisture-sensitive reagents were performed under a nitrogen atmosphere. ${ }^{1} \mathrm{H}$ NMR spectra were recorded at 300 and $500 \mathrm{MHz}$ using Bruker AV NMR spectrometer. ${ }^{13} \mathrm{C}$ NMR spectra were recorded at 75 and $125 \mathrm{MHz}$ using Bruker AV NMR spectrometer. The chemical shifts were reported as $\delta \mathrm{ppm}$ relative to TMS, using the residual solvent peak as the reference unless otherwise noted. All coupling constants $(J)$ are given in hertz. Data are reported as follows: chemical shift, multiplicity $(\mathrm{s}=$ singlet, $\mathrm{d}=$ doublet, $\mathrm{t}=$ triplet, $\mathrm{q}=$ quartet, $\mathrm{br}=$ broad, $\mathrm{m}=$ multiplet), number of protons and coupling constants. Thin layer chromatography was performed using Merck silica gel 60 F-254 thin layer plates, which were developed using one of the following techniques: UV fluorescence $(254 \mathrm{~nm})$, alkaline potassium permanganate solution $(0.5 \% \mathrm{w} / \mathrm{v})$ or ninhydrin $(0.2 \% \mathrm{w} / \mathrm{v})$ and iodine vapors. Automated flash column chromatography was carried out on prepacked silica cartridges using the indicated solvent system on Biotage Isolera chromatography system. Target compounds 33a, 34a-k, and 39a-d were crystallized in ethanol, solid was collected, washed with EtOAc and then hot solutions of 20-30\% EtOAc in hexanes to afford red to orange solids. If necessary, the products were purified with automated flash column chromatography. The chemical purity of target compounds was $\$ 95 \%$ determined by HPLC coupled to electrospray ionization mass spectrometry (LC/ESI-MS) analysis. LC-MS analyses and purity data of compounds were obtained using an Agilent 6130 quadrupole LC-MS instrument connected to an Agilent 1200 HPLC system, and both instruments were connected to an Agilent diode array detector. A C-18 reversed phase column (Vydac monomeric/Phenomenex/Kinetex $2.6 \mu \mathrm{m} \mathrm{XB-C18,50} \mathrm{mm} \times 4.6 \mathrm{~mm}$ ) was used as stationary phase, and water and methanol/acetonitrile (both containing 0.1-0.25\% TFA) were used as mobile phase (gradient 0-100\% methanol, flow $0.8 \mathrm{~mL} / \mathrm{min}$, run time $15 \mathrm{~min}$ ). UV absorbance at the fixed wavelength of $254 \mathrm{~nm}$ and positive and negative ESI-MS data were recorded. The retention time and corresponding ESI-MS data were used to identify molecules. HRMS data were obtained using Waters/Macromass LCT electrospray ionization (ESI) on a time-of-flight (TOF) mass spectrometer at the Mass Spectrometry Facility at Indiana University Chemistry Department (http://msf.chem.indiana.edu).

\section{Synthesis of $26 a$ and $26 b$}

\section{3-(3-Methyl-5-oxo-4,5-dihydro-1H-pyrazol-1-yl)benzoic Acid (26a)-Ethyl}

acetoacetate ( $2.01 \mathrm{~mL}, 1.2$ equiv) was added to a solution of 3-hydrazinobenzoic acid $\mathbf{2 5 a}$ ( 2 $\mathrm{g}, 1$ equiv) in glacial acetic acid $(30 \mathrm{~mL})$ under an argon atmosphere. After addition, the reaction mixture was heated at reflux with stirring for $12 \mathrm{~h}$. Once the reaction was allowed to cool to room temperature, the reaction mixture was concentrated in vacuo resulting in the formation of a precipitate. The solid was filtered and washed with 5\% $\mathrm{MeOH}$ in DCM (two 
times) and then two times with DCM to obtain 26a as an off-white solid (2.06 g, 72\% yield, requires no further purification). TLC: $4 \% \mathrm{MeOH}$ in DCM, $R_{f}=0.42$; visualized with UV. ${ }^{1} \mathrm{H}$ NMR (500 MHz, DMSO): $\delta 13.22$ (brs, $\left.1 \mathrm{H}, C O O H\right), 8.36$ (s, 1H), 8.05 (d, $1 \mathrm{H}, J=8.5$ $\mathrm{Hz}), 7.94(\mathrm{~d}, 1 \mathrm{H}, J=8.0 \mathrm{~Hz}), 7.70(\mathrm{t}, 1 \mathrm{H}, J=8.0$ and $16 \mathrm{~Hz}), 5.97(\mathrm{~s}, 1 \mathrm{H}), 2.45(\mathrm{~s}, 3 \mathrm{H}$, $\left.\mathrm{CH}_{3}\right) .{ }^{13} \mathrm{C}$ NMR (125 MHz, DMSO): $\delta 166.48,158.79,154.09,150.10,144.90,136.81$, 132.15, 129.96, 127.57, 124.16, 120.66, 104.64, 102.19, 19.12, 14.25. HRMS (ESI): calcd for $\mathrm{C}_{11} \mathrm{H}_{11} \mathrm{~N}_{2} \mathrm{O}_{3}[\mathrm{M}+\mathrm{H}]^{+} \mathrm{m} / \mathrm{Z}=219.0770$, found 219.0764 .

4-(3-Methyl-5-oxo-4,5-dihydro-1H-pyrazol-1-yl)benzoic Acid (26b)-26b was prepared by an above-described procedure using 4-hydrazinobenzoic acid hydrochloride $\mathbf{2 5 b}$ $(2 \mathrm{~g})$ as a starting material. Off-white solid $(1.76 \mathrm{~g}, 76 \%$ yield, requires no further purification). TLC: $4 \% \mathrm{MeOH}$ in DCM, $R_{f}=0.42$; visualized with UV. ${ }^{1} \mathrm{H} \mathrm{NMR}(300 \mathrm{MHz}$, DMSO): $\delta 12.87$ (brs, $1 \mathrm{H}, C O O H), 7.98$ (d, 2H, $J=8.8 \mathrm{~Hz}$ ), 7.88 (d, $2 \mathrm{H}, J=8.4 \mathrm{~Hz}$ ), 5.38 (s, $1 \mathrm{H}), 2.12$ (s, 3H, $\left.\mathrm{CH}_{3}\right) .{ }^{13} \mathrm{C}$ NMR (125 MHz, DMSO): $\delta 172.17,170.56,167.32,159.86$, 150.25, 142.66, 142.06, 130.80, 126.86, 126.46, 119.33, 117.49, 117.34, 43.61, 14.45.

HRMS (ESI): calcd for $\mathrm{C}_{11} \mathrm{H}_{9} \mathrm{~N}_{2} \mathrm{O}_{3}[\mathrm{M}-\mathrm{H}]^{-} \mathrm{m} / \mathrm{z}=217.0613$, found 217.0619.

\section{Synthesis of $27 \mathrm{a}$ and $27 \mathrm{~b}$}

Ethyl 3-(3-Methyl-5-oxo-4,5-dihydro-1H-pyrazol-1-yl)benzoate (27a)-To a stirred suspension of 3-(3-methyl-5-oxo-4,5-dihydro-1H-pyrazol-1-yl)benzoic acid 26a (1.95 g) in anhydrous ethanol $(30 \mathrm{~mL})$ was added a catalytic amount of concentrated sulfuric acid (1.5 $\mathrm{mL}$ ) slowly under an argon atmosphere. The reaction mixture was refluxed for $12 \mathrm{~h}$, and then it was allowed to cool to room temperature. The solvent was removed under vacuum, and the obtained residue was dissolved in ethyl acetate and washed successively with saturated $\mathrm{NaHCO}_{3}(2 \times 10 \mathrm{~mL})$, water, and brine solution. The organic layer was dried over $\mathrm{Na}_{2} \mathrm{SO}_{4}$ and concentrated under reduced pressure. The crude residue was purified by Biotage automated flash column chromatography using 0-50\% EtOAc in hexanes as the eluent to furnish ethyl 3-(3-methyl-5-oxo-4,5-dihydro- $1 H$-pyrazol-1-yl)benzoate 27a as a red oil ( $1.69 \mathrm{~g}, 77 \%$ yield). TLC: $45 \%$ EtOAc in hexanes, $R_{f}=0.44$; visualized with UV. ${ }^{1} \mathrm{H}$ NMR (300 MHz, $\left.\mathrm{CDCl}_{3}\right): \delta 8.41(\mathrm{~s}, 1 \mathrm{H}), 8.05(\mathrm{~d}, 1 \mathrm{H}, J=8.2 \mathrm{~Hz}), 7.78(\mathrm{~d}, 1 \mathrm{H}, J=8.0 \mathrm{~Hz})$, $7.38(\mathrm{t}, 1 \mathrm{H}, J=7.95$ and $15.99 \mathrm{~Hz}), 4.35-4.28\left(\mathrm{q}, 2 \mathrm{H}, O C H_{2}\right), 3.37\left(\mathrm{~s}, 2 \mathrm{H}, C_{2}\right), 2.11(\mathrm{~s}$, $\left.3 \mathrm{H}, \mathrm{CH}_{3}\right), 1.33$ (t, $3 \mathrm{H}, J=7.11$ and $\left.14.25 \mathrm{~Hz}, \mathrm{CH}_{3}\right) .{ }^{13} \mathrm{C} \mathrm{NMR}\left(75 \mathrm{MHz}, \mathrm{CDCl}_{3}\right): \delta 170.70$, $166.14,156.84,138.18,131.22,128.82,125.77,122.69,119.46,61.10,43.02,16.93,14.29$. MS (ESI) $\mathrm{m} / \mathrm{z}=247.1[\mathrm{M}+\mathrm{H}]^{+}$.

Ethyl 4-(3-Methyl-5-oxo-4,5-dihydro-1H-pyrazol-1-yl)benzoate (27b)-27b was prepared by an above-described procedure using $26 \mathbf{b}(1.60 \mathrm{~g})$ as a starting material. White solid (1.44 g, 80\% yield). TLC: $40 \%$ EtOAc in hexanes, $R_{f}=0.44$; visualized with UV. ${ }^{1} \mathrm{H}$ NMR (300 MHz, CDCl $\left.{ }_{3}\right): \delta 8.05(\mathrm{~d}, 2 \mathrm{H}, J=8.97 \mathrm{~Hz}), 8.01(\mathrm{~d}, 2 \mathrm{H}, J=8.94 \mathrm{~Hz}), 4.40-4.33$ (q, $\left.2 \mathrm{H}, \mathrm{OCH}_{2}\right), 3.46\left(\mathrm{~s}, 2 \mathrm{H}, \mathrm{CH}_{2}\right), 2.22\left(\mathrm{~s}, 3 \mathrm{H}, \mathrm{CH}_{3}\right), 1.39$ (t, $3 \mathrm{H}, J=7.11$ and $14.25 \mathrm{~Hz}$, $\left.\mathrm{CH}_{3}\right) .{ }^{13} \mathrm{C} \mathrm{NMR}\left(75 \mathrm{MHz}, \mathrm{CDCl}_{3}\right): \delta 170.78,166.18,156.89,141.69,130.54,126.42$, 117.61, 60.90, 43.17, 17.09, 14.36. MS (ESI) $\mathrm{m} / z=247.1[\mathrm{M}+\mathrm{H}]^{+}$. 


\section{Synthesis of 2-Chloro-5-(5-formylfuran-2-yl)benzoic Acid (30)}

A solution of $\mathrm{K}_{2} \mathrm{CO}_{3}$ (2.37 g, 3 equiv) in water $(10 \mathrm{~mL})$ was added to a mixture of 4chloro-3-carboxyphenylboronic acid $\mathbf{2 9}$ (1.37 g, 1.2 equiv) and 5-bromo-2-furaldehyde 28 (1 $\mathrm{g}, 1$ equiv) in toluene/ethanol $(1: 1, \mathrm{v} / \mathrm{v}, 60 \mathrm{~mL})$. The mixture was degassed with argon for 5 min, and then $\mathrm{Pd}\left(\mathrm{PPh}_{3}\right)_{4}$ (330 mg, 0.05 equiv) was added. The reaction mixture was stirred at $90{ }^{\circ} \mathrm{C}$ for $15 \mathrm{~h}$. The reaction mixture was cooled to room temperature, filtered through Celite, and washed with water $(2 \times 10 \mathrm{~mL})$. The $\mathrm{pH}$ of the solution was adjusted to $1-2$ by addition of $6 \mathrm{~N} \mathrm{HCl}$ solution. The precipitated reaction mixture was extracted with dichloromethane $(3 \times 100 \mathrm{~mL})$; the combined organic fractions were washed with brine, dried over anhydrous $\mathrm{Na}_{2} \mathrm{SO}_{4}$, and concentrated under reduced pressure. The crude product was triturated with 20-30\% EtOAc in hexanes ( 2 times), and solid was filtered to afford 2chloro-5-(5-formylfuran-2-yl)benzoic acid $\mathbf{3 0}$ (1.24 g, 87\% yield) as an off-white solid. TLC: $60 \%$ EtOAc in hexanes, $R_{f}=0.40$; visualized with $\mathrm{UV}$ and $\mathrm{KMnO}_{4}$ solution. ${ }^{1} \mathrm{H}$ NMR (300 MHz, DMSO): $\delta 13.74$ (brs, $1 \mathrm{H}, C O O H), 9.63(\mathrm{~s}, 1 \mathrm{H}, C H O), 8.23(\mathrm{~d}, 1 \mathrm{H}, J=2.22$ $\mathrm{Hz}), 8.01(\mathrm{dd}, 1 \mathrm{H}, J=2.28$ and $8.43 \mathrm{~Hz}), 7.70(\mathrm{~d}, 1 \mathrm{H}, J=8.34 \mathrm{~Hz}), 7.67(\mathrm{~d}, 1 \mathrm{H}, J=2.85$ $\mathrm{Hz}), 7.45$ (d, $1 \mathrm{H}, J=3.75 \mathrm{~Hz}) .{ }^{13} \mathrm{C}$ NMR (75 MHz, DMSO): $\delta 178.64,166.63,156.44$, $152.47,132.93,132.78,132.13,129.00,128.10,127.23,110.56$. MS (ESI) $\mathrm{m} / z=249.0[\mathrm{M}$ $-\mathrm{H}]^{-}$.

\section{Synthesis of $31 a$ and $31 b$}

(Z)-2-Chloro-5-(5-((1-(3-(ethoxycarbonyl)phenyl)-3-methyl-5-oxo-1Hpyrazol-4(5H)-ylidene)methyl)furan-2-yl)benzoic Acid (31a)-Ethyl 3-(3-methyl-5oxo-4,5-dihydro-1 $H$-pyrazol-1-yl)benzoate 27 a (1 g, 1 equiv) and 2-chloro-5-(5formylfuran-2-yl)benzoic acid $\mathbf{3 0}$ (1.01 g, 1 equiv) were dissolved in glacial acetic acid (50 $\mathrm{mL}$ ). The reaction mixture was heated at reflux with stirring for $3 \mathrm{~h}$. Solvent was removed in vacuo, and solid was suspended in EtOH, filtered, washed with EtOH, EtOAc, and DCM (2 times each) to obtain 31a as a red solid (1.48 $\mathrm{g}, 76 \%$ yield, requires no further purification). TLC: $5 \% \mathrm{MeOH}$ in DCM, $R_{f}=0.45$; visualized with UV. Major $Z$-isomer data: ${ }^{1} \mathrm{H}$ NMR (300 MHz, DMSO) $\delta 13.75$ (brs, $1 \mathrm{H}, C O O H), 8.63(\mathrm{~d}, 1 \mathrm{H}, J=3.87 \mathrm{~Hz}), 8.48(\mathrm{t}, 1 \mathrm{H}, J=$ 1.86 and $3.69 \mathrm{~Hz}), 8.27(\mathrm{~d}, 1 \mathrm{H}, J=2.22 \mathrm{~Hz}), 8.19(\mathrm{~d}, 1 \mathrm{H}, J=7.08 \mathrm{~Hz}), 8.01(\mathrm{dd}, 1 \mathrm{H}, J=$ 2.22 and $8.43 \mathrm{~Hz}), 7.76-7.64(\mathrm{~m}, 3 \mathrm{H}), 7.56-7.51(\mathrm{~m}, 2 \mathrm{H}), 4.36-4.29\left(\mathrm{q}, 2 \mathrm{H}, O C H_{2}\right), 2.64$ (s, $0.29 \mathrm{H}$, minor isomer, $\left.\mathrm{CH}_{3}\right), 2.32\left(\mathrm{~s}, 2.71 \mathrm{H}\right.$, major isomer, $\left.\mathrm{CH}_{3}\right), 1.33(\mathrm{t}, 3 \mathrm{H}, J=7.08$ and $\left.14.16 \mathrm{~Hz}, \mathrm{CH}_{3}\right) .{ }^{13} \mathrm{C}$ NMR $(75 \mathrm{MHz}$, DMSO): $\delta 166.59,165.88,162.11,157.65,151.64$, $150.82,138.96,133.15,132.67,131.11,130.91,130.08,129.77,128.96,127.84,127.26$, $125.17,122.41,121.60,118.43,112.91,61.38,14.65,13.29$. MS (ESI) $\mathrm{m} / z=477.1[\mathrm{M}-\mathrm{H}]$ - .

(Z)-2-Chloro-5-(5-((1-(4-(ethoxycarbonyl)phenyl)-3-methyl-5-oxo-1Hpyrazol-4(5H)-ylidene)methyl)furan-2-yl)benzoic Acid (31b)-31b was prepared by an above-described procedure using $\mathbf{2 7} \mathbf{b}$ ( $1 \mathrm{~g}, 1$ equiv) and $\mathbf{3 0}$ (1.01 g, 1 equiv) as starting materials. Red solid (1.69 g, $87 \%$ yield). TLC: $5 \% \mathrm{MeOH}$ in DCM, $R_{f}=0.48$; visualized with UV. Major $Z$-isomer data: ${ }^{1} \mathrm{H}$ NMR (300 MHz, DMSO) $\delta 13.72$ (brs, $1 \mathrm{H}, \mathrm{COOH}$ ), 8.62 $(\mathrm{d}, 1 \mathrm{H}, J=3.84 \mathrm{~Hz}), 8.33(\mathrm{~d}, 1 \mathrm{H}, J=2.19 \mathrm{~Hz}), 8.15-7.90(\mathrm{~m}, 5 \mathrm{H}), 7.79(\mathrm{~s}, 1 \mathrm{H}), 7.71(\mathrm{~d}, 1 \mathrm{H}$, $J=8.49 \mathrm{~Hz}), 7.58(\mathrm{~d}, 1 \mathrm{H}, J=3.84 \mathrm{~Hz}), 4.33-4.26\left(\mathrm{q}, 2 \mathrm{H}, O C H_{2}\right), 2.68(\mathrm{~s}, 0.51 \mathrm{H}$, minor isomer, $\mathrm{CH}_{3}$ ), 2.34 (s, $2.49 \mathrm{H}$, major isomer, $\mathrm{CH}_{3}$ ), 1.32 (t, $3 \mathrm{H}, \mathrm{J}=7.11$ and $14.19 \mathrm{~Hz}, \mathrm{CH}_{3}$ ). 
${ }^{13} \mathrm{C}$ NMR (75 MHz, DMSO): $\delta 166.60,165.69,162.41,157.81,152.26,150.83,142.42$, 133.23, 132.70, 132.18, 130.71, 129.03, 127.87, 127.35, 125.42, 121.39, 117.39, 112.97, 61.01, 14.67, 13.35. MS (ESI) $\mathrm{m} / z=477.1[\mathrm{M}-\mathrm{H}]^{-}$.

\section{Synthesis of Compounds 1 and 32}

(Z)-5-(5-((1-(3-Carboxyphenyl)-3-methyl-5-oxo-1H-pyrazol-4(5H)ylidene)methyl)furan-2-yl)-2-chlorobenzoic Acid (1)—To a stirred suspension of compound 31a $(150 \mathrm{mg})$ in THF/MeOH $(2: 1, \mathrm{v} / \mathrm{v}, 10 \mathrm{~mL})$ was added $2 \mathrm{~N} \mathrm{NaOH}(1 \mathrm{~mL})$ solution. The reaction mixture was stirred at room temperature for $6 \mathrm{~h}$. Solvent was removed in vacuo, and residue was acidified to $\mathrm{pH} 2-3$ using $20 \%$ citric acid solution. The product was extracted with EtOAc $(3 \times 15 \mathrm{~mL})$. The combined organic extracts were washed with brine, dried over $\mathrm{Na}_{2} \mathrm{SO}_{4}$, and concentrated under reduced pressure. The product was crystallized in EtOAc and triturated with 30\% EtOAc in hexanes to afford compound $\mathbf{1}$ (120 $\mathrm{mg}, 85 \%$ yield) as an orange solid. Isomer data: ${ }^{1} \mathrm{H}$ NMR (300 MHz, DMSO) $\delta 13.27$ (brs, $1 \mathrm{H}, C O O H), 12.88(\mathrm{brs}, 1 \mathrm{H}, C O O H), 8.61(\mathrm{~d}, 1 \mathrm{H}, J=3.7 \mathrm{~Hz}), 8.50$ (t, $1 \mathrm{H}, J=1.95$ and 3.85 $\mathrm{Hz}), 8.23(\mathrm{~d}, 1 \mathrm{H}, J=1.95 \mathrm{~Hz}), 8.20(\mathrm{~d}, 1 \mathrm{H}, J=7.35 \mathrm{~Hz}), 8.01(\mathrm{~m}, 1 \mathrm{H}), 7.76-7.66(\mathrm{~m}, 3 \mathrm{H})$, $7.58-7.53(\mathrm{~m}, 2 \mathrm{H}), 2.65$ (s, $0.77 \mathrm{H}$; minor isomer, $\left.\mathrm{CH}_{3}\right), 2.37$ (s, 2.23H; major isomer, $\mathrm{CH}_{3}$ ). MS (ESI) $\mathrm{m} / z=473.1[\mathrm{M}+\mathrm{Na}]^{+}$. HRMS (ESI): calcd for $\mathrm{C}_{23} \mathrm{H}_{13} \mathrm{~N}_{2} \mathrm{O}_{6} \mathrm{Cl}[\mathrm{M}-2 \mathrm{H}]^{-} \mathrm{m} / z=$ 448.0462, found 448.0469. HPLC purity: 95.36\%.

\section{(Z)-5-(5-((1-(4-Carboxyphenyl)-3-methyl-5-oxo-1H-pyrazol-4(5H)-} ylidene)methyl)furan-2-yl)-2-chlorobenzoic Acid (32)-32 was prepared by an above-described procedure using $\mathbf{3 1 b}(200 \mathrm{mg}$ ) as starting material. Orange solid (154 mg, $82 \%$ yield). ${ }^{1} \mathrm{H}$ NMR (300 MHz, DMSO): $\delta 13.19$ (brs, $1 \mathrm{H}, \mathrm{COOH}$ ), 12.84 (brs, $1 \mathrm{H}$, $\mathrm{COOH}), 8.67(\mathrm{~d}, 1 \mathrm{H}, J=3.84 \mathrm{~Hz}), 8.10-7.91(\mathrm{~m}, 6 \mathrm{H}), 7.80-7.77(\mathrm{~m}, 1 \mathrm{H}), 7.70(\mathrm{~d}, 1 \mathrm{H}, J=$ $8.64 \mathrm{~Hz}), 7.59-7.53(\mathrm{~m}, 1 \mathrm{H}), 2.68$ (s, $0.58 \mathrm{H}$; minor isomer, $\left.\mathrm{CH}_{3}\right), 2.34$ (s, $2.43 \mathrm{H}$; major isomer, $\mathrm{CH}_{3}$ ). MS (ESI) $\mathrm{m} / \mathrm{z}=448.1[\mathrm{M}-2 \mathrm{H}]^{-}$. HRMS (ESI): calcd for $\mathrm{C}_{23} \mathrm{H}_{14} \mathrm{~N}_{2} \mathrm{O}_{6} \mathrm{Cl}[\mathrm{M}$ $-\mathrm{H}]^{-} \mathrm{m} / \mathrm{z}=449.0540$, found 449.0547. HPLC purity: $95.13 \%$.

\section{General Synthesis of Amides 33a-j}

(Z)-Ethyl 3-(4-((5-(4-Chloro-3-((4-fluorobenzyl)carbamoyl)phenyl)furan-2-yl)methylene)-3-methyl-5-oxo-4,5-dihydro-1 H-pyrazol-1-yl)benzoate (33a)-To a solution of compound 31a (300 mg, 1 equiv) in dry DMF (6 mL) were added EDCI.HCl (180 mg, 1.5 equiv), HOBt (127 mg 1.5 equiv), and DIPEA ( $0.16 \mathrm{~mL}, 1.5$ equiv), and the mixture was stirred for $30 \mathrm{~min}$ at room temperature under an argon atmosphere. 4Fluorobenzylamine ( $75 \mu \mathrm{L}, 1.05$ equiv) and DIPEA $(0.16 \mathrm{~mL}, 1.5$ equiv) were added to the reaction mixture. The reaction mixture was stirred at room temperature for $18 \mathrm{~h}$. The reaction mixture was poured into water and extracted with EtOAc $(3 \times 20 \mathrm{~mL})$. The combined organic extracts were washed with saturated $\mathrm{NaHCO}_{3}(2 \times 10 \mathrm{~mL})$, brine, dried over $\mathrm{Na}_{2} \mathrm{SO}_{4}$, and concentrated under reduced pressure. The product was triturated with mixture of EtOAc in hexanes (2-3 times) to afford 33a (279 mg, 76\% yield) as a red solid. TLC: $3 \% \mathrm{MeOH}$ in DCM, $R_{f}=0.45$; visualized with UV. Isomer data: ${ }^{1} \mathrm{H}$ NMR (300 MHz, DMSO) $\delta 9.16(\mathrm{t}, 1 \mathrm{H}, J=5.52$ and $11.28 \mathrm{~Hz}), 8.66(\mathrm{~d}, 1 \mathrm{H}, J=3.72 \mathrm{~Hz}), 8.53(\mathrm{t}, 1 \mathrm{H}, J=1.5$ and $3.6 \mathrm{~Hz}$, major), $8.23(\mathrm{~d}, 1 \mathrm{H}, J=8.34 \mathrm{~Hz}), 8.03-7.92(\mathrm{~m}, 2 \mathrm{H}), 7.81-7.74(\mathrm{~m}, 2 \mathrm{H})$, $7.72-7.67$ (q, 1H), 7.62-7.54 (m, 2H), 7.43 (t, 2H, $J=8.43$ and $14.07 \mathrm{~Hz}), 7.24-7.16$ (m, 
2H), 4.49 (d, $\left.2 \mathrm{H}, J=5.73 \mathrm{~Hz}, \mathrm{NHCH}_{2}\right), 4.36-4.30\left(\mathrm{q}, 2 \mathrm{H}, \mathrm{OCH}_{2}\right), 2.64\left(\mathrm{~s}, 1.56 \mathrm{H}, \mathrm{CH}_{3}\right)$, 2.35 (s, $\left.1.44 \mathrm{H}, \mathrm{CH}_{3}\right), 1.34$ (t, 3H, $J=7.08$ and $\left.14.16 \mathrm{~Hz}, \mathrm{CH}_{3}\right) .{ }^{13} \mathrm{C} \mathrm{NMR}(75 \mathrm{MHz}$, DMSO): $\delta 166.24,165.92,162.23,158.06,151.81,150.81,139.01,138.22,135.56,131.55$, $131.33,131.17,130.25,129.84,129.74,128.04,127.85,127.34,125.51,125.33,122.67$, 121.57, 118.62, 115.69, 115.41, 112.91, 61.44, 42.34, 14.67, 13.34. MS (ESI) $\mathrm{m} / z=586.1$ $[\mathrm{M}+\mathrm{H}]^{+}$. HRMS (ESI): calcd for $\mathrm{C}_{32} \mathrm{H}_{26} \mathrm{~N}_{3} \mathrm{O}_{5} \mathrm{ClF}[\mathrm{M}+\mathrm{H}]^{+} \mathrm{m} / z=586.1545$, found 586.1548. HPLC purity: $98.73 \%$.

Compounds $\mathbf{3 3 b}-\mathbf{j}$ were synthesized by an above synthetic procedure described for the preparation of amide 33a using appropriate starting materials. Each compound was triturated with the mixture of EtOAc in hexanes (2-3 times) to afford desired compound.

(Z)-Ethyl 3-(4-((5-(4-Chloro-3-((3-methoxyphenyl)carbamoyl)-phenyl)furan-2yl)methylene)-3-methyl-5-oxo-4,5-dihydro-1H-pyrazol-1-yl)benzoate (33b)—Red solid (226 mg, $62 \%$ yield). TLC: $3 \% \mathrm{MeOH}$ in EtOAc, $R_{f}=0.47$; visualized with UV. Major $Z$-isomer data: ${ }^{1} \mathrm{H}$ NMR (300 MHz, DMSO) $\delta 10.64$ (s, $\left.1 \mathrm{H}, N H\right), 8.65(\mathrm{~d}, 1 \mathrm{H}, J=3.81 \mathrm{~Hz}$ ), $8.52(\mathrm{t}, 1 \mathrm{H}, J=1.83$ and $3.63 \mathrm{~Hz}), 8.22-8.14(\mathrm{~m}, 2 \mathrm{H}), 8.06(\mathrm{dd}, 1 \mathrm{H}, J=2.16$ and $8.46 \mathrm{~Hz})$, 7.81-7.70 (m, 3H), 7.64-7.53 (m, 2H), 7.43 (s, 1H), 7.29-7.27 (m, 2H), 6.74-6.70 (m, 1H), 4.37-4.30 (q, $2 \mathrm{H}, \mathrm{OCH}_{2}$ ), 3.75 (s, 3H, $\left.\mathrm{OCH}_{3}\right), 2.70$ (s, $0.58 \mathrm{H}$; minor isomer, $\left.\mathrm{CH}_{3}\right), 2.33$ (s, 2.42H; major isomer, $\left.\mathrm{CH}_{3}\right), 1.33$ (t, $3 \mathrm{H}, \mathrm{J}=7.11$ and $\left.14.19 \mathrm{~Hz}, \mathrm{CH}_{3}\right) .{ }^{13} \mathrm{C} \mathrm{NMR}(75 \mathrm{MHz}$, DMSO): $\delta 165.91,164.69,162.20,160.01,157.96,151.75,150.82,140.40,139.00,138.22$, $131.78,131.52,131.21,130.66,127.96,125.69,125.28,121.63,118.58,112.32,109.89$, 105.83, 61.42, 55.51, 14.66, 13.30. MS (ESI) $\mathrm{m} / z=584.1[\mathrm{M}+\mathrm{H}]^{+}$. HRMS (ESI): calcd for $\mathrm{C}_{32} \mathrm{H}_{26} \mathrm{~N}_{3} \mathrm{O}_{6} \mathrm{Cl}[\mathrm{M}]^{+} \mathrm{m} / \mathrm{z}=583.1510$, found 583.1524 .

(Z)-Ethyl 3-(4-((5-(4-Chloro-3-((3,4-dimethoxyphenyl)carbamoyl)phenyl)furan-2-yl)methylene)-3-methyl-5-oxo-4,5-dihydro-1Hpyrazol-1-yl)benzoate (33c)—Red solid (261 mg, 68\% yield). TLC: $3 \% \mathrm{MeOH}$ in EtOAc, $R_{f}=0.54$; visualized with UV. Major $Z$-isomer data: ${ }^{1} \mathrm{H}$ NMR (300 MHz, DMSO) $\delta$ $10.52(\mathrm{~s}, 1 \mathrm{H}, N H), 8.67(\mathrm{~d}, 1 \mathrm{H}, J=3.72 \mathrm{~Hz}), 8.56-8.50(\mathrm{~m}, 1 \mathrm{H}), 8.27-8.14(\mathrm{~m}, 2 \mathrm{H}), 8.06-$ $7.94(\mathrm{~m}, 1 \mathrm{H}), 7.82-7.73(\mathrm{~m}, 3 \mathrm{H}), 7.64-7.54(\mathrm{~m}, 2 \mathrm{H}), 7.48-7.44(\mathrm{~m}, 1 \mathrm{H}), 7.29-7.26$ (dd, $1 \mathrm{H}, J=2.16$ and $8.67 \mathrm{~Hz}), 6.97-6.94(\mathrm{~d}, 1 \mathrm{H}, J=8.82 \mathrm{~Hz}), 4.38-4.31\left(\mathrm{q}, 2 \mathrm{H}, O C H_{2}\right), 3.75$ (s, $6 \mathrm{H}, \mathrm{diOCH}$ ), 2.72 (s, $0.59 \mathrm{H}$; minor isomer, $\mathrm{CH}_{3}$ ), 2.34 (s, 2.41H; major isomer, $\mathrm{CH}_{3}$ ), $1.34\left(\mathrm{t}, 3 \mathrm{H}, J=7.08\right.$ and $\left.14.16 \mathrm{~Hz}, \mathrm{CH}_{3}\right) .{ }^{13} \mathrm{C}$ NMR (75 MHz, DMSO): $\delta 165.92,164.21$, $162.21,158.01,151.75,150.81,150.35,149.00,148.71,145.79,138.01,138.83,138.36$, $138.22,132.85,131.84,131.59,131.19,131.01,130.18,129.88,128.01,127.92,127.38$, $127.05,125.72,125.28,122.59,121.60,118.58,118.32,112.93,112.44,111.97,104.97$, $61.43,56.17,55.84,55.38,14.66,13.30$. MS (ESI) $\mathrm{m} / z=614.1[\mathrm{M}+\mathrm{H}]^{+}$. HRMS (ESI): calcd for $\mathrm{C}_{33} \mathrm{H}_{29} \mathrm{~N}_{3} \mathrm{O}_{7} \mathrm{Cl}[\mathrm{M}+\mathrm{H}]^{+} \mathrm{m} / z=614.1694$, found 614.1696.

(Z)-Ethyl 3-(4-((5-(4-Chloro-3-((cyclopropylmethyl)carbamoyl)-phenyl)furan-2yl)methylene)-3-methyl-5-oxo-4,5-dihydro-1H-pyrazol-1-yl)benzoate (33d)—Red solid (249 mg, $75 \%$ yield). TLC: $3 \% \mathrm{MeOH}$ in DCM, $R_{f}=0.43$; visualized with UV. Major $Z$-isomer data: ${ }^{1} \mathrm{H}$ NMR (500 MHz, DMSO) $\delta 8.68-8.64(\mathrm{~m}, 2 \mathrm{H}), 8.54(\mathrm{t}, 1 \mathrm{H}, J=1.8$ and $3.55 \mathrm{~Hz}), 8.23-8.20(\mathrm{~m}, 1 \mathrm{H}), 8.01-7.92(\mathrm{~m}, 2 \mathrm{H}), 7.78-7.74(\mathrm{~m}, 2 \mathrm{H}), 7.68(\mathrm{~d}, 1 \mathrm{H}, J=8.3$ $\mathrm{Hz}), 7.60-7.57$ (m, 2H), 4.38-4.33 (q, 2H, OCH $), 3.18\left(\mathrm{~m}, 2 \mathrm{H}, \mathrm{NHCH}_{2}\right), 2.72(\mathrm{~s}, 0.64 \mathrm{H}$; 
minor isomer, $\left.\mathrm{CH}_{3}\right), 2.35$ (s, $2.36 \mathrm{H}$; major isomer, $\left.\mathrm{CH}_{3}\right), 1.35$ (t, $3 \mathrm{H}, J=7.1$ and $14.2 \mathrm{~Hz}$, $\left.\mathrm{CH}_{3}\right), 1.06-1.0(\mathrm{~m}, 1 \mathrm{H}, \mathrm{CH}), 0.49-0.45\left(\mathrm{~m}, 2 \mathrm{H}, \mathrm{CH}_{2}\right), 0.28-0.25\left(\mathrm{~m}, 2 \mathrm{H}, \mathrm{CH}_{2}\right) .{ }^{13} \mathrm{C}$ NMR (125 MHz, DMSO): $\delta 165.51,165.44,161.74,157.65,151.27,150.29,138.54,138.14$, $131.07,130.56,129.68,129.37,127.54,127.29,126.66,124.96,124.80,112.16,121.05$, 118.16, 112.28, 60.92, 43.24, 14.17, 12.81, 10.65. MS (ESI) $\mathrm{m} / z=532.1[\mathrm{M}+\mathrm{H}]^{+}$. HRMS (ESI): calcd for $\mathrm{C}_{29} \mathrm{H}_{27} \mathrm{~N}_{3} \mathrm{O}_{5} \mathrm{Cl}[\mathrm{M}+\mathrm{H}]^{+} \mathrm{m} / \mathrm{z}=532.1639$, found 532.1640 .

(Z)-Ethyl 3-(4-((5-(4-Chloro-3-(cyclopropylcarbamoyl)phenyl)-furan-2yl)methylene)-3-methyl-5-oxo-4,5-dihydro-1H-pyrazol-1-yl)benzoate (33e)—Red solid (223 mg, 69\% yield). TLC: $3 \% \mathrm{MeOH}$ in DCM, $R_{f}=0.46$; visualized with UV. Major $Z$-isomer data: ${ }^{1} \mathrm{H}$ NMR $(300 \mathrm{MHz}, \mathrm{DMSO}) \delta 8.68(\mathrm{~d}, 1 \mathrm{H}, J=4.26 \mathrm{~Hz}), 8.63(\mathrm{~d}, 1 \mathrm{H}, J=3.6$ $\mathrm{Hz}), 8.52(\mathrm{~s}, 1 \mathrm{H}), 8.22(\mathrm{~d}, 1 \mathrm{H}, J=7.41 \mathrm{~Hz}), 7.97-7.88(\mathrm{~m}, 2 \mathrm{H}), 7.79-7.73(\mathrm{~m}, 2 \mathrm{H})$, 7.67-7.52 (m, 3H), 4.37-4.30 (q, 2H, OCH $), 2-88-2.82(\mathrm{~m}, 1 \mathrm{H}, C H) 2.68(\mathrm{~s}, 0.46 \mathrm{H}$; minor isomer, $\mathrm{CH}_{3}$ ), 2.35 (s, 2.54H; major isomer, $\left.\mathrm{CH}_{3}\right), 1.33$ (t, $3 \mathrm{H}, J=7.08$ and $14.13 \mathrm{~Hz}$, $\mathrm{CH}_{3}$ ), 0.75-0.69 (m, 2H, $\left.\mathrm{CH}_{2}\right), 0.58-0.53$ (m, 2H, $\left.\mathrm{CH}_{2}\right) .{ }^{13} \mathrm{C}$ NMR (75 MHz, DMSO): $\delta$ $167.19,165.92,162.20,158.09,151.76,150.75,139.02,138.83,138.34,138.06,131.04$, $130.17,129.88,128.05,127.75,127.16,125.51,125.27,122.59,121.50,118.58,112.83$, $61.42,23.23,14.67,13.33,6.16$. MS (ESI) $\mathrm{m} / z=518.1[\mathrm{M}+\mathrm{H}]^{+}$. HRMS (ESI): calcd for $\mathrm{C}_{28} \mathrm{H}_{25} \mathrm{~N}_{3} \mathrm{O}_{5} \mathrm{Cl}[\mathrm{M}+\mathrm{H}]^{+} \mathrm{m} / \mathrm{z}=518.1483$, found 518.1488.

\section{(Z)-Ethyl 3-(4-((5-(4-Chloro-3-((tetrahydro-2H-pyran-4-yl)-} carbamoyl)phenyl)furan-2-yl)methylene)-3-methyl-5-oxo-4,5-dihydro-1 $\mathrm{H}$ pyrazol-1-yl)benzoate (33f)—Red solid (246 mg, 70\% yield). TLC: $3 \% \mathrm{MeOH}$ in DCM, $R_{f}=0.45$; visualized with UV. Major $Z$-isomer data: ${ }^{1} \mathrm{H}$ NMR (300 MHz, DMSO) $\delta 8.69$ $(\mathrm{m}, 2 \mathrm{H}), 8.53(\mathrm{t}, 1 \mathrm{H}, J=1.71$ and $3.51 \mathrm{~Hz}), 8.26-8.20(\mathrm{~m}, 1 \mathrm{H}), 8.02-7.87(\mathrm{~m}, 2 \mathrm{H}), 7.79(\mathrm{~s}$, $1 \mathrm{H}), 7.75(\mathrm{~d}, 1 \mathrm{H}, J=8.1 \mathrm{~Hz}), 7.69-7.55(\mathrm{~m}, 3 \mathrm{H}), 4.38-4.31\left(\mathrm{q}, 2 \mathrm{H}, O_{C H}\right), 4.04-3.92(\mathrm{~m}$, $1 \mathrm{H}, \mathrm{CH}), 3.90-3.84\left(\mathrm{~m}, 2 \mathrm{H}, \mathrm{CH}_{2}\right), 3.45-3.39\left(\mathrm{~m}, 2 \mathrm{H}, \mathrm{CH}_{2}\right), 2.71$ (s, $0.58 \mathrm{H}$; minor isomer, $\left.\mathrm{CH}_{3}\right), 2.34$ (s, 2.42H; major isomer, $\left.\mathrm{CH}_{3}\right), 1.85-1.75\left(\mathrm{~m}, 2 \mathrm{H}, \mathrm{CH}_{2}\right), 1.60-1.45(\mathrm{~m}, 2 \mathrm{H}$, $\mathrm{CH}_{2}$ ), 1.33 (t, $3 \mathrm{H}, J=7.08$ and $14.01 \mathrm{~Hz}, \mathrm{CH}_{3}$ ). ${ }^{13} \mathrm{C}$ NMR (75 MHz, DMSO): $\delta 166.29$, 165.92, 165.37, 162.21, 158.13, 156.81, 152.40, 151.81, 150.77, 146.86, 141.35, 139.03, $138.55,138.40,131.41,131.02,130.59,130.24,127.93,127.78,127.16,125.33,124.64$, $122.65,121.49,118.62,110.44,66.29,61.44,46.06,32.62,14.67,13.36$. MS (ESI) $\mathrm{m} / z=$ $562.1[\mathrm{M}+\mathrm{H}]^{+}$. HRMS (ESI): calcd for $\mathrm{C}_{30} \mathrm{H}_{29} \mathrm{~N}_{3} \mathrm{O}_{6} \mathrm{Cl}[\mathrm{M}+\mathrm{H}]^{+} \mathrm{m} / z=562.1745$, found 562.1753.

(Z)-Ethyl 3-(4-((5-(4-Chloro-3-(((tetrahydro-2H-pyran-4-yl)methyl)carbamoyl)phenyl)furan-2-yl)methylene)-3-methyl-5-oxo-4,5dihydro-1H-pyrazol-1-yl)benzoate (33g)—Red solid (267 mg, 74\% yield). TLC: $3 \%$ $\mathrm{MeOH}$ in DCM, $R_{f}=0.45$; visualized with UV. Major $Z$-isomer data: ${ }^{1} \mathrm{H}$ NMR $(300 \mathrm{MHz}$, DMSO) $\delta 8.69-8.63(\mathrm{~m}, 2 \mathrm{H}), 8.53(\mathrm{~s}, 1 \mathrm{H}), 8.22(\mathrm{~d}, 1 \mathrm{H}, J=9.06 \mathrm{~Hz}), 7.99-7.90(\mathrm{~m}, 2 \mathrm{H})$, 7.81-7.74 (m, 1H), 7.77 (s, 1H), 7.67-7.53 (m, 3H), 4.38-4.28 (q, 2H, OCH $), 3.90-3.81$ (m, 2H, $\left.\mathrm{CH}_{2}\right), 3.32-3.22\left(\mathrm{~m}, 2 \mathrm{H}, \mathrm{CH}_{2}\right), 3.19-3.13\left(\mathrm{~m}, 2 \mathrm{H}, \mathrm{NHCH}_{2}\right), 2.72(\mathrm{~s}, 0.58 \mathrm{H}$; minor isomer, $\left.\mathrm{CH}_{3}\right), 2.34$ (s, 2.42H; major isomer, $\left.\mathrm{CH}_{3}\right), 1.84-1.71(\mathrm{~m}, 1 \mathrm{H}, \mathrm{CH}), 1.69-1.60(\mathrm{~m}$, $\left.2 \mathrm{H}, \mathrm{CH}_{2}\right), 1.33$ (t, 3H, $\mathrm{J}=7.08$ and $\left.13.95 \mathrm{~Hz}, \mathrm{CH}_{3}\right), 1.26-1.15\left(\mathrm{~m}, 2 \mathrm{H}, \mathrm{CH}_{2}\right) .{ }^{13} \mathrm{C} \mathrm{NMR}(75$ MHz, DMSO): $\delta 178.58,166.65,166.24,165.92,162.22,158.13,156.80,152.40,151.79$, 
$150.77,146.85,141.35,139.02,138.68,138.52,131.72,131.02,130.60,127.95,127.88$, $127.79,125.30,124.65,122.65,121.51,119.60,112.86,110.42,108.52,67.22,61.43$, 45.27, 36.25, 31.22, 14.69, 13.43. MS (ESI) $\mathrm{m} / \mathrm{z}=576.2[\mathrm{M}+\mathrm{H}]^{+}$. HRMS (ESI): calcd for $\mathrm{C}_{31} \mathrm{H}_{31} \mathrm{~N}_{3} \mathrm{O}_{6} \mathrm{Cl}[\mathrm{M}+\mathrm{H}]^{+} \mathrm{m} / z=576.1901$, found 576.1913 .

(Z)-Ethyl 4-(4-((5-(4-Chloro-3-((4-fluorobenzyl)carbamoyl)-phenyl)furan-2yl)methylene)-3-methyl-5-oxo-4,5-dihydro-1H-pyrazol-1-yl)benzoate (33h)—Red solid (300 mg, 79\% yield). TLC: $3 \% \mathrm{MeOH}$ in DCM, $R_{f}=0.47$; visualized with UV. Major $Z$-isomer data: ${ }^{1} \mathrm{H}$ NMR (300 MHz, DMSO) $\delta 9.17(\mathrm{t}, 1 \mathrm{H}, J=5.88$ and $11.76 \mathrm{~Hz}), 8.62(\mathrm{~s}$, $1 \mathrm{H}), 8.16-7.97(\mathrm{~m}, 6 \mathrm{H}), 7.75(\mathrm{~s}, 1 \mathrm{H}), 7.69(\mathrm{~d}, 1 \mathrm{H}, J=8.4 \mathrm{~Hz}), 7.60$ (d, $1 \mathrm{H}, J=3.33 \mathrm{~Hz})$, 7.45-7.41 (m, 2H), 7.22-7.18 (m, 2H), $4.49\left(\mathrm{~d}, 2 \mathrm{H}, J=5.76 \mathrm{~Hz}, N H C H_{2}\right), 4.32-4.25(\mathrm{q}$, $\left.2 \mathrm{H}, \mathrm{OCH}_{2}\right), 2.62$ (s, $0.74 \mathrm{H}$; minor isomer, $\left.\mathrm{CH}_{3}\right), 2.33$ (s, 2.26H; major isomer, $\left.\mathrm{CH}_{3}\right), 1.31$ (t, 3H, $J=7.08$ and $\left.14.1 \mathrm{~Hz}, \mathrm{CH}_{3}\right) .{ }^{13} \mathrm{C}$ NMR (75 MHz, DMSO): $\delta 166.23,166.07,165.23$, $162.53,158.66,158.19,154.90,151.00,150.74,150.37,149.06,144.85,142.46,138.22$, $138.04,135.57,131.61,131.34,130.78,130.35,129.73,127.99,127.83,126.52,125.53$, 124.10, 121.33, 118.28, 117.52, 115.91, 115.69, 115.41, 112.89, 61.05, 44.84, 14.69, 13.47. MS (ESI) $\mathrm{m} / z=586.1[\mathrm{M}+\mathrm{H}]^{+}$. HRMS (ESI): calcd for $\mathrm{C}_{32} \mathrm{H}_{26} \mathrm{~N}_{3} \mathrm{O}_{5} \mathrm{ClF}[\mathrm{M}+\mathrm{H}]^{+} \mathrm{m} / z=$ 586.1545 , found 586.1549 .

(Z)-Ethyl 4-(4-((5-(4-Chloro-3-((3-methoxyphenyl)carbamoyl)-phenyl)furan-2yl)methylene)-3-methyl-5-oxo-4,5-dihydro-1H-pyrazol-1-yl)benzoate (33i)—Dark red solid (272 mg, $67 \%$ yield). TLC: $3 \% \mathrm{MeOH}$ in EtOAc, $R_{f}=0.49$; visualized with UV. Major $Z$-isomer data: ${ }^{1} \mathrm{H}$ NMR (300 MHz, DMSO) $\delta 10.66(\mathrm{~s}, 1 \mathrm{H}, N H), 8.60(\mathrm{~d}, 1 \mathrm{H}, J=3.0$ $\mathrm{Hz}), 8.20(\mathrm{~d}, 1 \mathrm{H}, J=1.5 \mathrm{~Hz}), 8.14-7.94(\mathrm{~m}, 5 \mathrm{H}), 7.82-7.73(\mathrm{~m}, 2 \mathrm{H}), 7.63-7.54(\mathrm{~m}, 1 \mathrm{H})$, $7.45-7.39$ (m/brs, 1H), 7.29-7.23 (m, 2H), 6.74-6.69 (m, 1H), 4.323-4.26 (q, 2H, OCH $)$, 3.75 (s, 3H, $\left.\mathrm{OCH}_{3}\right), 2.70$ (s, $072 . \mathrm{H}$; minor isomer, $\left.\mathrm{CH}_{3}\right), 2.32$ (s, $2.31 \mathrm{H}$; major isomer, $\mathrm{CH}_{3}$ ), 1.32 (t, $3 \mathrm{H}, \mathrm{J}=7.05$ and $14.04 \mathrm{~Hz}, \mathrm{CH}_{3}$ ). ${ }^{13} \mathrm{C}$ NMR (75 MHz, DMSO): $\delta 165.70$, $164.69,162.43,160.01,158.07,152.30,150.79,150.44,142.44,140.41,138.20,131.58$, $131.22,130.74,130.16,129.06,127.92,125.76,125.46,121.36,117.42,112.97,112.31$, 109.89, 105.82, 61.04, 55.51, 55.38, 54.95, 14.69, 13.34. MS (ESI) $\mathrm{m} / z=584.1[\mathrm{M}+\mathrm{H}]^{+}$. HRMS (ESI): calcd for $\mathrm{C}_{30} \mathrm{H}_{21} \mathrm{~N}_{3} \mathrm{O}_{6} \mathrm{Cl}[\mathrm{M}-\mathrm{H}]^{-} \mathrm{m} / z=584.1588$, found 584.1596 .

(Z)-Ethyl 4-(4-((5-(4-Chloro-3-((cyclopropylmethyl)carbamoyl)-phenyl)furan-2yl)methylene)-3-methyl-5-oxo-4,5-dihydro-1H-pyrazol-1-yl)benzoate (33j)—Red solid (364 mg, $82 \%$ yield). TLC: $3 \% \mathrm{MeOH}$ in DCM, $R_{f}=0.44$; visualized with UV. Major $Z$-isomer data: ${ }^{1} \mathrm{H}$ NMR (300 MHz, DMSO) $\delta 8.74(\mathrm{t}, 1 \mathrm{H}, J=5.31$ and $10.5 \mathrm{~Hz}), 8.59(\mathrm{~s}$, $1 \mathrm{H}), 8.07(\mathrm{t}, 2 \mathrm{H}, J=8.73$ and $17.7 \mathrm{~Hz}), 8.0(\mathrm{~d}, 4 \mathrm{H}, J=7.83 \mathrm{~Hz}), 7.75(\mathrm{~s}, 1 \mathrm{H}), 7.66(\mathrm{~d}, 1 \mathrm{H}, J$ $=8.85 \mathrm{~Hz}), 7.58(\mathrm{~d}, 1 \mathrm{H}, J=3.96 \mathrm{~Hz}), 4.32-4.25\left(\mathrm{q}, 2 \mathrm{H}, O C H_{2}\right), 3.16(\mathrm{t}, 2 \mathrm{H}, J=6.09$ and $12.42 \mathrm{~Hz}, \mathrm{NHCH}_{2}$ ), 2.68 (s, $0.66 \mathrm{H}$; minor isomer, $\left.\mathrm{CH}_{3}\right), 2.32$ (s, 2.34H; major isomer, $\left.\mathrm{CH}_{3}\right), 1.31$ (t, $3 \mathrm{H}, \mathrm{J}=7.11$ and $\left.14.16 \mathrm{~Hz}, \mathrm{CH}_{3}\right), 1.09-0.98(\mathrm{~m}, 1 \mathrm{H}, \mathrm{CH}), 0.48-0.42(\mathrm{~m}, 2 \mathrm{H}$, $\mathrm{CH}_{2}$ ), 0.28-0.23 (m, 2H, $\mathrm{CH}_{2}$ ). ${ }^{13} \mathrm{C}$ NMR (75 MHz, DMSO): $\delta 166.00,166.07,165.92$, $162.49,158.74,157.92,152.39,150.77,150.34,148.97,142.47,138.63,138.42,131.62$, 131.12, 130.77, 127.95, 127.73, 127.20, 125.50, 121.07, 119.60, 117.51, 112.81, 61.06, 43.68, 14.70, 13.39, 11.17, 3.36. MS (ESI) $\mathrm{m} / z=532.1[\mathrm{M}+\mathrm{H}]^{+}$. HRMS (ESI): calcd for $\mathrm{C}_{29} \mathrm{H}_{27} \mathrm{~N}_{3} \mathrm{O}_{5} \mathrm{Cl}[\mathrm{M}+\mathrm{H}]^{+} \mathrm{m} / \mathrm{z}=532.1639$, found 532.1642 . 


\section{General Synthesis of Target Compounds 34a-d and 34f-k}

(Z)-3-(4-((5-(4-Chloro-3-((4-fluorobenzyl)carbamoyl)phenyl)furan-2yl)methylene)-3-methyl-5-oxo-4,5-dihydro-1H-pyrazol-1-yl)-benzoic Acid (34a)

- To a stirred suspension of ester 33a ( $80 \mathrm{mg}, 1$ equiv) in THF/EtOH/ $\mathrm{H}_{2} \mathrm{O}(4: 2: 1,7 \mathrm{~mL})$ was added $\mathrm{LiOH}$ ( $32 \mathrm{mg}, 10$ equiv). The reaction mixture was stirred at room temperature for $12 \mathrm{~h}$. Solvent was removed in vacuo, and residue was acidified to $\mathrm{pH} 2-3$ using $20 \%$ citric acid solution. The product was extracted with EtOAc $(3 \times 15 \mathrm{~mL})$. The combined organic extracts were washed with brine, dried over $\mathrm{Na}_{2} \mathrm{SO}_{4}$, and concentrated under reduced pressure. The product was crystallized in $\mathrm{EtOH}$, and solid was collected, washed with EtOAc and then hot solutions of 20-30\% EtOAc in hexanes to afford target compound 34a (47 mg, 62\% yield) as a red solid. Major $Z$-isomer data: ${ }^{1} \mathrm{H}$ NMR (500 MHz, DMSO) $\delta$ $9.15(\mathrm{t}, 1 \mathrm{H}, J=5.8$ and $11.7 \mathrm{~Hz}), 8.68(\mathrm{~d}, 1 \mathrm{H}, J=3.05 \mathrm{~Hz}), 8.56(\mathrm{t}, 1 \mathrm{H}, J=1.7$ and 3.85 $\mathrm{Hz}), 8.20(\mathrm{~d}, 1 \mathrm{H}, J=7.45 \mathrm{~Hz}), 8.04-7.94(\mathrm{~m}, 2 \mathrm{H}), 7.82-7.69(\mathrm{~m}, 2 \mathrm{H}), 7.71(\mathrm{t}, 1 \mathrm{H}, J=8.15$ and $16.45 \mathrm{~Hz}), 7.62-7.55(\mathrm{~m}, 2 \mathrm{H}), 7.45-7.42(\mathrm{~m}, 2 \mathrm{H}), 7.22-7.17(\mathrm{~m}, 2 \mathrm{H}), 4.49(\mathrm{~d}, 2 \mathrm{H}, J=$ $5.85 \mathrm{~Hz}, \mathrm{NHCH}_{2}$ ), 2.68 (s, $0.76 \mathrm{H}$; minor isomer, $\mathrm{CH}_{3}$ ), 2.36 (s, 2.24H; major isomer, $\mathrm{CH}_{3}$ ). MS (ESI) $\mathrm{m} / z=556.1[\mathrm{M}-\mathrm{H}]^{-}$. HRMS (ESI): calcd for $\mathrm{C}_{30} \mathrm{H}_{20} \mathrm{~N}_{3} \mathrm{O}_{5} \mathrm{ClF}[\mathrm{M}-\mathrm{H}]^{-} \mathrm{m} / z=$ 556.1076, found 556.1079. HPLC purity: 97.24\%.

Target compounds 34b-d and 34f-k were synthesized by an above synthetic procedure described for the preparation of compound 34a using appropriate starting materials. Each compound was crystallized in EtOH, and solid was collected, washed with EtOAc and then hot solutions of 20-30\% EtOAc in hexanes to afford desired final compound. If necessary, the products were purified using 2-5\% $\mathrm{MeOH}$ in DCM (1\% AcOH in DCM) solvent system on automated flash column chromatography.

(Z)-3-(4-((5-(4-Chloro-3-((3-methoxyphenyl)carbamoyl)phenyl)-furan-2yl)methylene)-3-methyl-5-oxo-4,5-dihydro-1H-pyrazol-1-yl)benzoic Acid (34b)Red solid (111 mg, 69\% yield). Major $Z$-isomer data: ${ }^{1} \mathrm{H}$ NMR (300 MHz, DMSO) $\delta 13.04$ (brs, $1 \mathrm{H}, C O O H), 10.65(\mathrm{~s}, 1 \mathrm{H}, N H), 8.69$ (d, $1 \mathrm{H}, J=3.16 \mathrm{~Hz}), 8.55$ (t, $1 \mathrm{H}, J=1.95$ and 3.5 $\mathrm{Hz}), 8.31-8.19(\mathrm{~m}, 2 \mathrm{H}), 8.08-7.97(\mathrm{~m}, 1 \mathrm{H}), 7.80-7.70(\mathrm{~m}, 3 \mathrm{H}), 7.65-7.55(\mathrm{~m}, 2 \mathrm{H}), 7.43(\mathrm{~s}$, $1 \mathrm{H}), 7.29-7.28(\mathrm{~m}, 2 \mathrm{H}), 6.74-6.69(\mathrm{~m}, 1 \mathrm{H}), 3.76\left(\mathrm{~s}, 3 \mathrm{H}, O_{C H}\right), 2.73(\mathrm{~s}, 0.51 \mathrm{H}$; minor isomer, $\mathrm{CH}_{3}$ ), 2.34 (s, 2.49H; major isomer, $\left.\mathrm{CH}_{3}\right) .{ }^{13} \mathrm{C}$ NMR (75 MHz, DMSO): $\delta 172.50$, 167.51, 167.28, 165.08, 164.69, 162.22, 160.01, 157.94, 151.59, 140.41, 138.93, 132.03, 131.93, 131.22, 130.72, 129.72, 129.47, 125.48, 124.69, 122.30, 121.74, 112.32, 109.89, 105.83, 55.52, 13.30. MS (ESI) $\mathrm{m} / z=554.1[\mathrm{M}-\mathrm{H}]^{-}$. HRMS (ESI): calcd for $\mathrm{C}_{30} \mathrm{H}_{21} \mathrm{~N}_{3} \mathrm{O}_{6} \mathrm{Cl}[\mathrm{M}-\mathrm{H}]^{-} \mathrm{m} / \mathrm{z}=554.1119$, found 554.1124. HPLC purity: 98.63\%.

(Z)-3-(4-((5-(4-Chloro-3-((3,4-dimethoxyphenyl)carbamoyl)-phenyl)furan-2yl)methylene)-3-methyl-5-oxo-4,5-dihydro-1H-pyrazol-1-yl)benzoic Acid (34c)Red solid (60 mg, 63\% yield). Major $Z$-isomer data: ${ }^{1} \mathrm{H}$ NMR (300 MHz, DMSO) $\delta 13.14$ (brs, $1 \mathrm{H}, C O O H), 10.52(\mathrm{~s}, 1 \mathrm{H}, N H), 8.70(\mathrm{~d}, 1 \mathrm{H}, J=3.75 \mathrm{~Hz}), 8.56-8.51(\mathrm{~m}, 1 \mathrm{H})$, 8.28-8.15 (m, 2H), 8.08-7.95 (m, 1H), 7.80-7.74 (m, 3H), 7.65-7.55 (m, 2H), 7.47-7.43 (m, 1H), 7.30-7.27 (m, 1H), 6.97-6.90 (m, 1H), 3.75 and $3.74(\mathrm{~s}, 6 \mathrm{H}, \mathrm{diOCH}), 2.74(\mathrm{~s}$, 0.49H; minor isomer, $\mathrm{CH}_{3}$ ), 2.35 (s, $2.49 \mathrm{H}$; major isomer, $\left.\mathrm{CH}_{3}\right) .{ }^{13} \mathrm{C} \mathrm{NMR}(75 \mathrm{MHz}$, DMSO): $\delta 167.51,164.22,164.69,162.22,157.99,151.70,150.84,149.00,145.79,138.93$, 
$138.37,131.93,131.58,130.46,129.74,127.94,127.39,125.73,125.43,122.30,121.71$, 118.96, 112.44, 111.98, 104.97, 105.83, 56.17, 55.84, 13.30. MS (ESI) $\mathrm{m} / \mathrm{z}=584.1[\mathrm{M}-\mathrm{H}]$ -. HRMS (ESI): calcd for $\mathrm{C}_{31} \mathrm{H}_{23} \mathrm{~N}_{3} \mathrm{O}_{7} \mathrm{Cl}[\mathrm{M}-\mathrm{H}]^{-} \mathrm{m} / \mathrm{z}=584.1225$, found 584.1229. HPLC purity: $95.07 \%$.

(Z)-3-(4-((5-(4-Chloro-3-((cyclopropylmethyl)carbamoyl)phenyl)-furan-2yl)methylene)-3-methyl-5-oxo-4,5-dihydro-1H-pyrazol-1-yl)benzoic Acid (34d)Red solid (56 mg, 60\% yield). Major $Z$-isomer data: ${ }^{1} \mathrm{H}$ NMR (300 MHz, DMSO) $\delta 13.10$ (brs, 1H, COOH), 8.71-8.65 (m, 2H), $8.55(\mathrm{~s}, 1 \mathrm{H}), 8.20(\mathrm{~d}, 1 \mathrm{H}, J=7.74 \mathrm{~Hz}), 8.02-7.94(\mathrm{~m}$, $2 \mathrm{H}), 7.83-7.66(\mathrm{~m}, 3 \mathrm{H}), 7.62-7.53(\mathrm{~m}, 2 \mathrm{H}), 3.19-3.13\left(\mathrm{~m}, 2 \mathrm{H}, \mathrm{NHCH}_{2}\right), 2.73(\mathrm{~s}, 0.71 \mathrm{H}$; minor isomer, $\mathrm{CH}_{3}$ ), 2.35 (s, 2.29H; major isomer, $\left.\mathrm{CH}_{3}\right), 1.07-0.95$ (m, $\left.1 \mathrm{H}, \mathrm{CH}\right), 0.48-0.42$ (m, 2H, $\mathrm{CH}_{2}$ ), 0.28-0.23 (m, 2H, $\left.\mathrm{CH}_{2}\right) .{ }^{13} \mathrm{C}$ NMR (75 MHz, DMSO): $\delta 167.50,166.00$, $162.22,158.11,151.71,150.80,138.94,138.63,131.93,131.54,130.07,129.73,128.01$, $127.78,127.15,125.49,122.31,121.61,118.97,112.81,43.71,13.32,11.16,3.73$. MS (ESI) $\mathrm{m} / \mathrm{z}=502.1[\mathrm{M}-\mathrm{H}]^{-}$. HRMS (ESI): calcd for $\mathrm{C}_{27} \mathrm{H}_{21} \mathrm{~N}_{3} \mathrm{O}_{5} \mathrm{Cl}[\mathrm{M}-\mathrm{H}]^{-} \mathrm{m} / z=502.1170$, found 502.1172. HPLC purity: $97.11 \%$.

(Z)-3-(4-((5-(4-Chloro-3-(cyclopropylcarbamoyl)phenyl)furan-2-yl)methylene)-3methyl-5-oxo-4,5-dihydro-1H-pyrazol-1-yl)benzoic Acid (34f)—Red solid (64 mg, $68 \%$ yield). Major $Z$-isomer data: ${ }^{1} \mathrm{H}$ NMR (300 MHz, DMSO) $\delta 13.12$ (brs, $1 \mathrm{H}, \mathrm{COOH}$ ), 8.66-8.63 (m, 2H), $8.54(\mathrm{t}, 1 \mathrm{H}, J=1.77$ and $3.54 \mathrm{~Hz}), 8.20(\mathrm{~d}, 1 \mathrm{H}, J=8.16 \mathrm{~Hz}), 8.00-7.94$ $(\mathrm{m}, 2 \mathrm{H}), 7.82-7.73(\mathrm{~m}, 2 \mathrm{H}), 7.69-7.64(\mathrm{~m}, 1 \mathrm{H}), 7.60-7.53(\mathrm{~m}, 2 \mathrm{H}), 2.89-2.80(\mathrm{~m}, 1 \mathrm{H}$, $\mathrm{CH}), 2.71$ (s, $1.10 \mathrm{H}$; minor isomer, $\left.\mathrm{CH}_{3}\right), 2.34$ (s, $1.90 \mathrm{H}$; major isomer, $\left.\mathrm{CH}_{3}\right), 0.77-0.69(\mathrm{~m}$, $\left.2 \mathrm{H}, \mathrm{CH}_{2}\right), 0.58-0.53\left(\mathrm{~m}, 2 \mathrm{H}, \mathrm{CH}_{2}\right) .{ }^{13} \mathrm{C}$ NMR (75 MHz, DMSO): $\delta 167.50,167.19,162.21$, 158.06, 151.69, 150.78, 138.94, 138.38, 131.93, 131.57, 131.06, 130.15, 129.73, 127.99, $127.78,127.15,126.49,122.29,121.62,118.95,112.81,23.22,13.32,6.18$. MS (ESI) $\mathrm{m} / \mathrm{z}=$ $488.1[\mathrm{M}-\mathrm{H}]^{-}$. HRMS (ESI): calcd for $\mathrm{C}_{26} \mathrm{H}_{19} \mathrm{~N}_{3} \mathrm{O}_{5} \mathrm{Cl}[\mathrm{M}-\mathrm{H}]^{-} \mathrm{m} / \mathrm{z}=488.1013$, found 488.1017. HPLC purity: $95.74 \%$.

(Z)-3-(4-((5-(4-Chloro-3-((tetrahydro-2H-pyran-4-yl)carbamoyl)-phenyl)furan-2yl)methylene)-3-methyl-5-oxo-4,5-dihydro-1H-pyrazol-1-yl)benzoic Acid (34g)Red solid (72 mg, 76\% yield). Major $Z$-isomer data: ${ }^{1} \mathrm{H}$ NMR (300 MHz, DMSO) $\delta 13.11$ (brs, $1 \mathrm{H}, C O O H), 8.65-8.59(\mathrm{~m}, 2 \mathrm{H}), 8.54(\mathrm{t}, 1 \mathrm{H}, J=1.71$ and $3.42 \mathrm{~Hz}), 8.20(\mathrm{~d}, 1 \mathrm{H}, J=$ $8.16 \mathrm{~Hz}), 8.01-7.93(\mathrm{~m}, 2 \mathrm{H}), 7.82-7.73(\mathrm{~m}, 2 \mathrm{H}), 7.70-7.65(\mathrm{~m}, 1 \mathrm{H}), 7.61-7.53(\mathrm{~m}, 2 \mathrm{H})$, 4.05-3.95 (m, 1H, CH), 3.91-3.84 (m, 2H, $\left.\mathrm{CH}_{2}\right), 3.46-3.41\left(\mathrm{~m}, 2 \mathrm{H}, \mathrm{CH}_{2}\right), 2.72(\mathrm{~s}, 0.77 \mathrm{H}$; minor isomer, $\left.\mathrm{CH}_{3}\right), 2.34$ (s, $2.23 \mathrm{H}$; major isomer, $\left.\mathrm{CH}_{3}\right), 1.86-1.79\left(\mathrm{~m}, 2 \mathrm{H}, \mathrm{CH}_{2}\right)$, 1.59-1.46 (m, 2H, $\left.\mathrm{CH}_{2}\right) .{ }^{13} \mathrm{C}$ NMR (75 MHz, DMSO): $\delta 167.50,165.37,162.19,158.07$, $151.68,150.79,138.94,138.57,131.92,131.56,131.03,130.13,129.71,128.02,127.80$, $127.14,125.34,122.29,121.60,118.96,112.81,66.28,46.05,32.65,13.32 . \mathrm{MS}(\mathrm{ESI}) \mathrm{m} / \mathrm{z}=$ $532.1[\mathrm{M}-\mathrm{H}]^{-}$. HRMS (ESI): calcd for $\mathrm{C}_{28} \mathrm{H}_{23} \mathrm{~N}_{3} \mathrm{O}_{6} \mathrm{Cl}[\mathrm{M}-\mathrm{H}]^{-} \mathrm{m} / z=532.1275$, found 532.1281. HPLC purity: $96.33 \%$.

(Z)-3-(4-((5-(4-Chloro-3-(((tetrahydro-2H-pyran-4-yl)methyl)carbamoyl)phenyl)furan-2-yl)methylene)-3-methyl-5-oxo-4,5-dihydro-1Hpyrazol-1-yl)benzoic Acid (34h)—Red solid (66 mg, 70\% yield). Major $Z$-isomer data: 
${ }^{1} \mathrm{H}$ NMR (300 MHz, DMSO) $\delta 13.12$ (brs, $\left.1 \mathrm{H}, \mathrm{COOH}\right), 8.65-8.59(\mathrm{~m}, 2 \mathrm{H}), 8.54(\mathrm{~s}, 1 \mathrm{H})$, $8.19(\mathrm{~d}, 1 \mathrm{H}, J=7.77 \mathrm{~Hz}), 8.01-7.92(\mathrm{~m}, 2 \mathrm{H}), 7.82-7.73(\mathrm{~m}, 2 \mathrm{H}), 7.69-7.65(\mathrm{~m}, 1 \mathrm{H})$, 7.60-7.54 (m, 2H), 3.90-3.81 (m, 2H, $\left.C_{2}\right), 3.28-3.22\left(\mathrm{~m}, 2 \mathrm{H}, \mathrm{CH}_{2}\right), 3.19-3.13(\mathrm{~m}, 2 \mathrm{H}$, $\mathrm{NHCH}_{2}$ ), 2.70 (s, $0.67 \mathrm{H}$; minor isomer, $\mathrm{CH}_{3}$ ), 2.34 (s, 2.33H; major isomer, $\mathrm{CH}_{3}$ ), 1.84-1.72 (m, 1H, CH), 1.69-1.61 (m, 2H, $\left.\mathrm{CH}_{2}\right), 1.30-1.14\left(\mathrm{~m}, 2 \mathrm{H}, \mathrm{CH}_{2}\right) .{ }^{13} \mathrm{C}$ NMR $(75$ MHz, DMSO): $\delta 167.00,165.75,161.70,157.56,151.18,150.29,138.42,138.18,131.41$, $130.93,130.54,129.66,129.22,127.47,127.31,126.60,124.98,121.79,121.10,118.45$, 112.32, 66.72, 44.78, 34.77, 30.40, 12.80. MS (ESI) $\mathrm{m} / z=546.1[\mathrm{M}-\mathrm{H}]^{-}$. HRMS (ESI): calcd for $\mathrm{C}_{29} \mathrm{H}_{25} \mathrm{~N}_{3} \mathrm{O}_{6} \mathrm{Cl}[\mathrm{M}-\mathrm{H}]^{-} \mathrm{m} / \mathrm{z}=546.1432$, found 546.1434. HPLC purity: $97.82 \%$.

\section{(Z)-4-(4-((5-(4-Chloro-3-((4-fluorobenzyl)carbamoyl)phenyl)-furan-2-} yl)methylene)-3-methyl-5-oxo-4,5-dihydro-1H-pyrazol-1-yl)benzoic Acid (34i).Red solid (66 mg, 70\% yield). Isomer data: ${ }^{1} \mathrm{H}$ NMR (300 MHz, DMSO) $\delta 12.85$ (brs, $1 \mathrm{H}$, $\mathrm{COOH}), 9.17(\mathrm{t}, 1 \mathrm{H}, J=5.88$ and $11.79 \mathrm{~Hz}), 8.64(\mathrm{~d}, 1 \mathrm{H}, J=3.66 \mathrm{~Hz}), 8.10-7.92(\mathrm{~m}, 6 \mathrm{H})$, 7.83-7.79 (m, 1H), 7.71 (dd, $1 \mathrm{H}, J=2.67$ and $8.4 \mathrm{~Hz}), 7.62-7.56(\mathrm{~m}, 1 \mathrm{H}), 7.45-7.40(\mathrm{~m}$, 2H), 7.23-7.15 (m, 2H), $4.48\left(\mathrm{~d}, 2 \mathrm{H}, J=5.88 \mathrm{~Hz}, N H C H_{2}\right), 2.66\left(\mathrm{~s}, 1.65 \mathrm{H}, \mathrm{CH}_{3}\right), 2.34(\mathrm{~s}$, $\left.1.35 \mathrm{H}, \mathrm{CH}_{3}\right) .{ }^{13} \mathrm{C}$ NMR (75 MHz, DMSO): $\delta 167.31,166.23,166.11,165.28,162.44$, $160.10,159.56,158.15,152.22,150.81,150.33,149.13,142.20,142.00,138.22,138.04$, 135.56, 131.83, 131.59, 131.47, 131.17, 131.03, 130.93, 127.83, 127.73, 126.52, 125.90, 125.54, 121.41, 119.71, 117.47, 117.25, 115.69, 115.42, 112.93, 42.32, 13.37. MS (ESI) $\mathrm{m} / \mathrm{z}$ $=556.1[\mathrm{M}-\mathrm{H}]^{-}$. HRMS (ESI): calcd for $\mathrm{C}_{30} \mathrm{H}_{20} \mathrm{~N}_{3} \mathrm{O}_{5} \mathrm{ClF}[\mathrm{M}-\mathrm{H}]^{-} \mathrm{m} / \mathrm{z}=556.1076$, found 556.1077 . HPLC purity: $98.93 \%$.

\section{(Z)-4-(4-((5-(4-Chloro-3-((3-methoxyphenyl)carbamoyl)phenyl)-furan-2-} yl)methylene)-3-methyl-5-oxo-4,5-dihydro-1H-pyrazol-1-yl)benzoic Acid (34j)Red solid (60 mg, 63\% yield). Major $Z$-isomer data: ${ }^{1} \mathrm{H}$ NMR (300 MHz, DMSO) $\delta 12.86$ (brs, 1H, COOH), $10.55(\mathrm{~s}, 1 \mathrm{H}, \mathrm{NH}), 8.14-7.96(\mathrm{~m}, 4 \mathrm{H}), 7.95-7.85(\mathrm{~m}, 3 \mathrm{H}), 7.83-7.75(\mathrm{~m}$, $1 \mathrm{H}), 7.73-7.66(\mathrm{~m}, 1 \mathrm{H}), 7.61-7.54(\mathrm{~m}, 1 \mathrm{H}), 7.47-7.40(\mathrm{~m}, 1 \mathrm{H}), 7.32-7.23(\mathrm{~m}, 2 \mathrm{H})$, 6.75-6.67 (m, 1H), 3.74 (s, 3H, $\left.\mathrm{OCH}_{3}\right), 2.74\left(\mathrm{~s}, 0.24 \mathrm{H}\right.$; minor isomer, $\left.\mathrm{CH}_{3}\right), 2.35$ (s, 2.87H; major isomer, $\left.\mathrm{CH}_{3}\right) .{ }^{13} \mathrm{C}$ NMR (75 MHz, DMSO): $\delta 167.21,165.10,164.66,162.20$, $159.98,157.91,150.39,140.46,137.86,132.01,131.58,130.86,130.07,129.92,128.42$, $127.51,125.52,123.55,122.26,119.96,119.68,112.32,109.79,105.80,55.47,13.36$. MS (ESI) $\mathrm{m} / \mathrm{z}=554.1[\mathrm{M}-\mathrm{H}]^{-}$. HRMS (ESI): calcd for $\mathrm{C}_{30} \mathrm{H}_{21} \mathrm{~N}_{3} \mathrm{O}_{6} \mathrm{Cl}[\mathrm{M}-\mathrm{H}]^{-} \mathrm{m} / \mathrm{z}=$ 554.1119, found 554.1122. HPLC purity: 96.34\%.

\section{(Z)-4-(4-((5-(4-Chloro-3-((cyclopropylmethyl)carbamoyl)phenyl)-furan-2-} yl)methylene)-3-methyl-5-oxo-4,5-dihydro-1H-pyrazol-1-yl)benzoic Acid (34k)Red solid (89 mg, 63\% yield). Major $Z$-isomer data: ${ }^{1} \mathrm{H}$ NMR (300 MHz, DMSO) $\delta 12.83$ (brs, $1 \mathrm{H}, \mathrm{COOH}$ ), 8.67 (t, $1 \mathrm{H}, J=5.1$ and $10.2 \mathrm{~Hz}$ ), $8.60(\mathrm{~s}, 1 \mathrm{H}), 8.06(\mathrm{t}, 2 \mathrm{H}, J=8.01$ and $14.19 \mathrm{~Hz}), 8.0(\mathrm{~d}, 4 \mathrm{H}, J=8.55 \mathrm{~Hz}), 7.76(\mathrm{~s}, 1 \mathrm{H}), 7.67(\mathrm{~d}, 1 \mathrm{H}, J=8.64 \mathrm{~Hz}), 7.58(\mathrm{~d}, 1 \mathrm{H}, J=$ $3.9 \mathrm{~Hz}$ ), 3.17 (t, $2 \mathrm{H}, J=5.7$ and $\left.11.4 \mathrm{~Hz}, \mathrm{NHCH}_{2}\right), 2.70$ (s, $0.81 \mathrm{H}$; minor isomer, $\left.\mathrm{CH}_{3}\right), 2.33$ (s, 2.19H; major isomer, $\left.\mathrm{CH}_{3}\right), 1.08-0.94(\mathrm{~m}, 1 \mathrm{H}, \mathrm{CH}), 0.49-0.40\left(\mathrm{~m}, 2 \mathrm{H}, \mathrm{CH}_{2}\right), 0.28-0.22$ (m, 2H, $\left.\mathrm{CH}_{2}\right) .{ }^{13} \mathrm{C}$ NMR (75 MHz, DMSO): $\delta 167.31,166.01,165.91,162.41,159.64$, $158.22,152.18,150.76,150.29,149.09,142.21,138.60,138.39,131.83,131.05,130.21$, $128.18,127.74,127.16,126.44,125.45,121.33,119.62,117.542,112.81,43.71,13.35$, 
11.17, 3.73. MS (ESI) $\mathrm{m} / z=502.1[\mathrm{M}-\mathrm{H}]^{-}$. HRMS (ESI): calcd for $\mathrm{C}_{27} \mathrm{H}_{21} \mathrm{~N}_{3} \mathrm{O}_{5} \mathrm{Cl}[\mathrm{M}-$ $\mathrm{H}]^{-} \mathrm{m} / z=502.1170$, found 502.1171. HPLC purity: $95.18 \%$.

\section{Synthesis of Target Compound 34e.}

(Z)-2-Chloro-N-(cyclopropylmethyl)-5-(5-((1-(3-((cyclopropylmethyl)carbamoyl)phenyl)-3methyl-5-oxo-1H-pyrazol-4(5H)-ylidene)methyl)furan-2-yl)-benzamide (34e)

34e was synthesized using an above synthetic procedure described for the preparation of compound 33a using 34d as a starting material. Product was crystallized in EtOH, and solid was collected, washed with EtOAc and then hot solutions of 20-30\% EtOAc in hexanes to afford 34e as a red solid (51 mg, 78\% yield). TLC: $4 \% \mathrm{MeOH}$ in DCM, $R_{f}=0.42$; visualized with UV. Major Zisomer data: ${ }^{1} \mathrm{H}$ NMR (300 MHz, DMSO) $\delta 8.70-8.65(\mathrm{~m}, 3 \mathrm{H})$, $8.36(\mathrm{~s}, 1 \mathrm{H}), 8.11(\mathrm{~d}, 1 \mathrm{H}, J=7.83 \mathrm{~Hz}), 7.99-7.91(\mathrm{~m}, 2 \mathrm{H}), 7.77(\mathrm{~s}, 1 \mathrm{H}), 7.68-7.65(\mathrm{~m}, 2 \mathrm{H})$, $7.58(\mathrm{~d}, 1 \mathrm{H}, J=3.9 \mathrm{~Hz}), 7.54-7.48(\mathrm{~m}, 1 \mathrm{H}), 3.19-3.13\left(\mathrm{q}, 4 \mathrm{H}, 2 \mathrm{NHCH}_{2}\right), 2.72(\mathrm{~s}, 0.56 \mathrm{H}$; minor isomer, $\left.\mathrm{CH}_{3}\right), 2.34$ (s, 2.44H; major isomer, $\left.\mathrm{CH}_{3}\right), 1.07-0.95(\mathrm{~m}, 2 \mathrm{H}, 2 \mathrm{CH}), 0.49-$ $0.41\left(\mathrm{~m}, 4 \mathrm{H}, 2 \mathrm{CH}_{2}\right), 0.27-0.23\left(\mathrm{~m}, 4 \mathrm{H}, 2 \mathrm{CH}_{2}\right) .{ }^{13} \mathrm{C} \mathrm{NMR}$ (75 MHz, DMSO): $\delta 166.30$, $166.01,162.14,159.49,158.05,151.50,150.80,138.76,138.61,136.01,131.51,131.23$, 131.07, 130.05, 129.23, 127.91, 127.78, 125.41, 123.34, 121.66, 120.89, 117.69, 112.76, 44.08, 43.71 13.31, 11.49, 11.16, $3.73(\mathrm{t})$. MS (ESI) $\mathrm{m} / z=557.1[\mathrm{M}+\mathrm{H}]^{+}$. HRMS (ESI): calcd for $\mathrm{C}_{31} \mathrm{H}_{30} \mathrm{~N}_{4} \mathrm{O}_{4} \mathrm{Cl}[\mathrm{M}+\mathrm{H}]^{+} \mathrm{m} / z=557.1956$, found 557.1957.

\section{Synthesis of 4-(5-Formylfuran-2-yl)benzoic Acid (36)}

Aldehyde 36 was synthesized using the Suzuki coupling reaction described for the preparation of compound $\mathbf{3 0}$ using 4-carboxyphenylboronic acid 35 as a starting material. The workup of this reaction was different from previous reaction as the product was insoluble in organic solvents after acidification. The reaction mixture was cooled to room temperature, and the solvent was removed under reduced pressure. The $\mathrm{pH}$ of the suspension was adjusted to $1-2$ by the addition of $6 \mathrm{~N} \mathrm{HCl}$ solution. The precipitated product was filtered, washed successively with water $(3 \times 15 \mathrm{~mL})$, EtOAc $(2 \times 10 \mathrm{~mL})$, DCM, and dried under high vacuum overnight to get 4-(5-formylfuran-2-yl)benzoic acid 36 (1.11 g, 90\% yield) as a white solid. ${ }^{1} \mathrm{H}$ NMR (500 MHz, DMSO): $\delta 13.16(\mathrm{~s}, 1 \mathrm{H}, C O O H), 9.66(\mathrm{~s}, 1 \mathrm{H}$, $C H O), 8.05(\mathrm{dd}, 2 \mathrm{H}, J=1.5$ and $6.5 \mathrm{~Hz}), 8.00(\mathrm{dd}, 2 \mathrm{H}, J=2.0$ and $7.0 \mathrm{~Hz}), 7.70(\mathrm{~d}, 1 \mathrm{H}, J=$ $3.5 \mathrm{~Hz}), 7.46(\mathrm{~d}, 1 \mathrm{H}, J=4.0 \mathrm{~Hz}) .{ }^{13} \mathrm{C} \mathrm{NMR}(125 \mathrm{MHz}, \mathrm{DMSO}): \delta 178.70,167.17,157.36$, 152.66, 132.78, 131.73, 130.61, 125.47, 111.05. MS (ESI) $m / z=215.1[\mathrm{M}-\mathrm{H}]^{-}$. HRMS (ESI): calcd for $\mathrm{C}_{12} \mathrm{H}_{7} \mathrm{O}_{4}[\mathrm{M}-\mathrm{H}]^{-} \mathrm{m} / z=215.0344$, found 215.0351 .

\section{Synthesis of Compounds $37 \mathrm{a}$ and $37 \mathrm{~b}$}

37a and 37b were prepared using an above Knoevenagel condensation reaction procedure described for the preparation of compound 31a using 4-(5-formylfuran-2-yl)benzoic acid 36 and $27 \mathbf{a}(600 \mathrm{mg})$ and $27 \mathbf{b}(600 \mathrm{mg})$, respectively, as starting materials. 
(Z)-4-(5-((1-(3-(Ethoxycarbonyl)phenyl)-3-methyl-5-oxo-1H-pyrazol-4(5H)ylidene)methyl)furan-2-yl)benzoic Acid (37a)

Red solid (844 mg, 78\% yield). Major $Z$-isomer data: ${ }^{1} \mathrm{H}$ NMR (500 MHz, DMSO) $\delta 13.14$ $(\mathrm{s}, 1 \mathrm{H}, C O O H), 8.63(\mathrm{~d}, 1 \mathrm{H}, J=3.5 \mathrm{~Hz}), 8.49(\mathrm{t}, 1 \mathrm{H}, J=1.5$ and $3.5 \mathrm{~Hz}), 8.20(\mathrm{~d}, 1 \mathrm{H}, J=$ $8.5 \mathrm{~Hz}), 8.03-7.90(\mathrm{~m}, 4 \mathrm{H}), 7.76-7.71(\mathrm{~m}, 1 \mathrm{H}), 7.65(\mathrm{~s}, 1 \mathrm{H}), 7.56-7.51(\mathrm{~m}, 2 \mathrm{H}), 4.35-4.29$ (q, $\left.2 \mathrm{H}, \mathrm{OCH}_{2}\right), 2.67$ (s, $0.68 \mathrm{H}$; minor isomer, $\left.\mathrm{CH}_{3}\right), 2.32$ (s, $2.32 \mathrm{H}$; major isomer, $\mathrm{CH}_{3}$ ), 1.34 (t, 3H, $J=7.5$ and $\left.14.5 \mathrm{~Hz}, C H_{3}\right) .{ }^{13} \mathrm{C}$ NMR (125 MHz, DMSO): $\delta 166.62,165.40$, $161.60,158.05,151.10,150.51,138.48,133.15,131.98,131.25,130.09,129.45,129.29$, 127.32, 124.91, 124.70, 121.27, 118.02, 112.91, 60.88, 14.15, 12.78. MS (ESI) $\mathrm{m} / \mathrm{z}=443.1$ $[\mathrm{M}-\mathrm{H}]^{-}$. HRMS (ESI): calcd for $\mathrm{C}_{25} \mathrm{H}_{19} \mathrm{~N}_{2} \mathrm{O}_{6}[\mathrm{M}-\mathrm{H}]^{-} \mathrm{m} / \mathrm{z}=443.1243$, found 443.1254.

\section{(Z)-4-(5-((1-(4-(Ethoxycarbonyl)phenyl)-3-methyl-5-oxo-1H-pyrazol-4(5H)- ylidene)methyl)furan-2-yl)benzoic Acid (37b)}

Red solid (920 mg, 85\% yield). Major $Z$-isomer data: ${ }^{1} \mathrm{H}$ NMR (300 MHz, DMSO) $\delta 13.08$ (brs, 1H, COOH), $8.56(\mathrm{~s}, 1 \mathrm{H}), 7.98-7.81(\mathrm{~m}, 7 \mathrm{H}), 7.62-7.39(\mathrm{~m}, 3 \mathrm{H}), 4.27-4.20(\mathrm{q}, 2 \mathrm{H}$, $\left.\mathrm{OCH}_{2}\right), 2.59$ (s, $0.68 \mathrm{H}$; minor isomer, $\left.\mathrm{CH}_{3}\right), 2.28\left(\mathrm{~s}, 2.32 \mathrm{H}\right.$; major isomer, $\left.\mathrm{CH}_{3}\right), 1.28$ (t, $3 \mathrm{H}, J=6.63$ and $12.57 \mathrm{~Hz}, \mathrm{CH}_{3}$ ). MS (ESI) $\mathrm{m} / \mathrm{z}=443.1[\mathrm{M}-\mathrm{H}]^{-}$. HRMS (ESI): calcd for $\mathrm{C}_{25} \mathrm{H}_{19} \mathrm{~N}_{2} \mathrm{O}_{6}[\mathrm{M}-\mathrm{H}]^{-} \mathrm{m} / \mathrm{z}=443.1243$, found 443.1249 .

\section{Synthesis of Amides 38a-d}

Compounds 38a,b and 38c,d were prepared using an above synthetic procedure described for the preparation of compound 33a using 37a (300 mg) and 37b (300 mg), respectively, and corresponding amines as a starting material.

\section{(Z)-Ethyl 3-(4-((5-(4-((4-Fluorobenzyl)carbamoyl)phenyl)furan-2-yl)methylene)-3-methyl-5- oxo-4,5-dihydro-1H-pyrazol-1-yl)-benzoate (38a).}

Red solid (294 mg, 79\% yield). Major $Z$-isomer data: ${ }^{1} \mathrm{H}$ NMR (300 MHz, DMSO) $\delta 9.29$ $(\mathrm{t}, 1 \mathrm{H}, J=5.7$ and $11.7 \mathrm{~Hz}), 8.64(\mathrm{~d}, 1 \mathrm{H}, J=3.72 \mathrm{~Hz}), 8.56-8.47(\mathrm{~m}, 1 \mathrm{H}), 8.21(\mathrm{~d}, 1 \mathrm{H}, J=$ $8.1 \mathrm{~Hz}), 8.06-7.86(\mathrm{~m}, 4 \mathrm{H}), 7.76-7.61(\mathrm{~m}, 2 \mathrm{H}), 7.57-7.44(\mathrm{~m}, 2 \mathrm{H}), 7.42-7.32(\mathrm{~m}, 2 \mathrm{H})$, $7.20-7.12(\mathrm{~m}, 2 \mathrm{H}), 4.45\left(\mathrm{~d}, 2 \mathrm{H}, J=5.67 \mathrm{~Hz}, \mathrm{NHCH}_{2}\right), 4.36-4.27$ (q, 2H, $\left.O C H_{2}\right), 2.67$ (s, $0.49 \mathrm{H}$; minor isomer, $\left.\mathrm{CH}_{3}\right), 2.32$ (s, $2.51 \mathrm{H}$; major isomer, $\left.\mathrm{CH}_{3}\right), 1.35-1.29\left(\mathrm{~m}, 3 \mathrm{H}, \mathrm{CH}_{3}\right)$. ${ }^{13} \mathrm{C}$ NMR (75 MHz, DMSO): $\delta 166.59,166.05,162.49,159.25,158.43,151.15,150.67$, $147.18,139.31,136.55,135.55,132.60,131.50,131.28,130.89,130.04129 .94,129.01$, $128.69,128.34,125.75,125.61,122.86,121.76,119.92,118.85,115.84,115.75,113.36$, 61.70 (d), 42.74, 14.99, 13.76 (d). MS (ESI) $\mathrm{m} / \mathrm{z}=552.1[\mathrm{M}+\mathrm{H}]^{+}$. HRMS (ESI): calcd for $\mathrm{C}_{32} \mathrm{H}_{27} \mathrm{~N}_{3} \mathrm{O}_{5} \mathrm{~F}[\mathrm{M}+\mathrm{H}]^{+} \mathrm{m} / \mathrm{z}=552.1935$, found 552.1939.

\section{(Z)-Ethyl 3-(4-((5-(4-((Cyclopropylmethyl)carbamoyl)phenyl)-furan-2-yl)methylene)-3- methyl-5-oxo-4,5-dihydro-1H-pyrazol-1-yl)benzoate (38b)}

Red solid (248 mg, 74\% yield). Major $Z$-isomer data: ${ }^{1} \mathrm{H}$ NMR (300 MHz, DMSO) $\delta$ 8.71-8.64 (m, 2H), $8.52(\mathrm{t}, 1 \mathrm{H}, J=1.8$ and $3.6 \mathrm{~Hz}), 8.21(\mathrm{~d}, 1 \mathrm{H}, J=8.22 \mathrm{~Hz}), 8.03-7.91(\mathrm{~m}$, $4 \mathrm{H}), 7.78-7.70(\mathrm{~m}, 2 \mathrm{H}), 7.59-7.50(\mathrm{~m}, 2 \mathrm{H}), 4.37-4.28\left(\mathrm{q}, 2 \mathrm{H}, O C H_{2}\right), 3.16(\mathrm{t}, 2 \mathrm{H}, J=6.21$ and $\left.12.36 \mathrm{~Hz}, \mathrm{NHCH}_{2}\right), 2.70\left(\mathrm{~s}, 0.71 \mathrm{H}\right.$; minor isomer, $\left.\mathrm{CH}_{3}\right), 2.34(\mathrm{~s}, 2.29 \mathrm{H}$; major isomer, $\mathrm{CH}_{3}$ ), 1.36-1.30 (m, 3H, $\left.\mathrm{CH}_{3}\right), 1.08-0.98(\mathrm{~m}, 1 \mathrm{H}, \mathrm{CH}), 0.47-0.41$ (m, 2H, $\left.\mathrm{CH}_{2}\right), 0.26-0.21$ 
(m, 2H, $\left.\mathrm{CH}_{2}\right) .{ }^{13} \mathrm{C}$ NMR (75 MHz, DMSO): $\delta 165.91,165.64,165.01,162.18,159.00$, $151.69,150.82,139.02,138.84,135.64,131.01,130.97,130.09,129.83,128.56,128.04$, 127.00, 125.25, 122.53, 121.43, 118.53, 112.96, 61.40, 44.11, 14.66, 13.31, 11.46, 3.83. MS (ESI) $\mathrm{m} / \mathrm{z}=498.1[\mathrm{M}+\mathrm{H}]^{+}$. HRMS (ESI): calcd for $\mathrm{C}_{29} \mathrm{H}_{28} \mathrm{~N}_{3} \mathrm{O}_{5}[\mathrm{M}+\mathrm{H}]^{+} \mathrm{m} / \mathrm{z}=$ 498.2029, found 498.2030.

\section{(Z)-Ethyl 4-(4-((5-(4-((4-Fluorobenzyl)carbamoyl)phenyl)furan-2-yl)methylene)-3-methyl-5- oxo-4,5-dihydro-1H-pyrazol-1-yl)-benzoate (38c)}

Red solid (297 mg, 80\% yield). Major $Z$-isomer data: ${ }^{1} \mathrm{H}$ NMR (300 MHz, DMSO) $\delta 9.20$ $(\mathrm{m}, 1 \mathrm{H}), 8.64(\mathrm{~d}, 1 \mathrm{H}, J=3.84 \mathrm{~Hz}), 8.11-7.92(\mathrm{~m}, 8 \mathrm{H}), 7.73(\mathrm{~d}, 1 \mathrm{H}, J=11.7 \mathrm{~Hz}), 7.58-7.49$ (m, 1H), 7.40-7.31 (m, 2H), 7.19-7.09 (m, 2H), 4.48-4.42 (q, 2H, NHCH $), 4.32-4.21(\mathrm{~m}$, $2 \mathrm{H}, \mathrm{OCH}_{2}$ ), 2.66 (s, $0.56 \mathrm{H}$; minor isomer, $\mathrm{CH}_{3}$ ), 2.34 (s, 2.44H; major isomer, $\mathrm{CH}_{3}$ ), 1.33-1.25 (m, 3H, $\left.\mathrm{CH}_{3}\right)$. MS (ESI) $\mathrm{m} / z=552.1[\mathrm{M}+\mathrm{H}]^{+}$. HRMS (ESI): calcd for $\mathrm{C}_{32} \mathrm{H}_{27} \mathrm{~N}_{3} \mathrm{O}_{5} \mathrm{~F}[\mathrm{M}+\mathrm{H}]^{+} \mathrm{m} / \mathrm{z}=552.1935$, found 552.1943.

\section{(Z)-Ethyl 4-(4-((5-(4-((Cyclopropylmethyl)carbamoyl)phenyl)-furan-2-yl)methylene)-3- methyl-5-oxo-4,5-dihydro-1H-pyrazol-1-yl)benzoate (38d)}

Red solid (251 mg, 75\% yield). Major $Z$-isomer data: ${ }^{1} \mathrm{H}$ NMR (300 MHz, DMSO) $\delta 8.69$ $(\mathrm{t}, 1 \mathrm{H}, J=5.58$ and $11.55 \mathrm{~Hz}), 8.63(\mathrm{~d}, 1 \mathrm{H}, J=3.66 \mathrm{~Hz}), 8.09-7.90(\mathrm{~m}, 8 \mathrm{H}), 7.70(\mathrm{~s}, 1 \mathrm{H})$, 7.56-7.50 (m, 1H), 4.31-4.22 (q, 2H, OCH $), 3.15\left(\mathrm{t}, 2 \mathrm{H}, J=5.7\right.$ and $12.0 \mathrm{~Hz}, \mathrm{NHCH}_{2}$ ), 2.68 (s, $0.70 \mathrm{H}$; minor isomer, $\left.\mathrm{CH}_{3}\right), 2.33$ (s, $2.30 \mathrm{H}$; major isomer, $\left.\mathrm{CH}_{3}\right), 1.33-127(\mathrm{~m}, 3 \mathrm{H}$, $\left.\mathrm{CH}_{3}\right), 1.08-0.99(\mathrm{~m}, 1 \mathrm{H}, \mathrm{CH}), 0.47-0.41\left(\mathrm{~m}, 2 \mathrm{H}, \mathrm{CH}_{2}\right), 0.26-0.21\left(\mathrm{~m}, 2 \mathrm{H}, \mathrm{CH}_{2}\right) . \mathrm{MS}$ (ESI) $\mathrm{m} / z=498.1[\mathrm{M}+\mathrm{H}]^{+}$. HRMS (ESI): calcd for $\mathrm{C}_{29} \mathrm{H}_{28} \mathrm{~N}_{3} \mathrm{O}_{5}[\mathrm{M}+\mathrm{H}]^{+} \mathrm{m} / z=498.2029$, found 498.2034 .

\section{Synthesis of Target Compounds 39a-d}

Target compounds 39a-d were synthesized by an above synthetic procedure described for the preparation of compound 34a using appropriate starting materials. Each compound was crystallized in EtOH, and solid was collected, washed with EtOAc and then hot solutions of 20-30\% EtOAc in hexanes to afford the desired final compound. If necessary, the products were purified using 2-5\% MeOH in DCM (1\% AcOH in DCM) solvent system on automated flash column chromatography.

(Z)-3-(4-((5-(4-((4-Fluorobenzyl)carbamoyl)phenyl)furan-2-yl)-methylene)-3-methyl-5oxo-4,5-dihydro-1H-pyrazol-1-yl)benzoic Acid (39a)

Red solid (103 mg, 73\% yield). Major $Z$-isomer data: ${ }^{1} \mathrm{H}$ NMR (300 MHz, DMSO) $\delta 13.12$ (s, $1 \mathrm{H}, \mathrm{COOH}), 9.20-9.16(\mathrm{~m}, 1 \mathrm{H}), 8.69(\mathrm{~d}, 1 \mathrm{H}, J=3.84 \mathrm{~Hz}), 8.55$ (t, $1 \mathrm{H}, J=1.83$ and 3.6 $\mathrm{Hz}$, major isomer), $8.21(\mathrm{~d}, 1 \mathrm{H}, J=8.16 \mathrm{~Hz}), 8.08-7.98(\mathrm{~m}, 4 \mathrm{H}), 7.83-7.73(\mathrm{~m}, 2 \mathrm{H})$, $7.60-7.53(\mathrm{~m}, 2 \mathrm{H}), 7.40-7.35(\mathrm{~m}, 2 \mathrm{H}), 7.16(\mathrm{t}, 2 \mathrm{H}, J=8.94$ and $17.82 \mathrm{~Hz}), 4.48(\mathrm{~d}, 2 \mathrm{H}, J=$ $5.79 \mathrm{~Hz}, \mathrm{NHCH}_{2}$ ), 2.75 (s, $1.06 \mathrm{H}$; minor isomer, $\mathrm{CH}_{3}$ ), 2.35 (s, $1.94 \mathrm{H}$; major isomer, $\mathrm{CH}_{3}$ ). ${ }^{13} \mathrm{C}$ NMR (75 MHz, DMSO): $\delta 167.03,165.34,162.76,161.74,159.56,158.43,149.97$, $148.23,138.46,135.68,134.79,131.44,130.79,129.32,129.22$, 128.19, 127.51, 125.04, $124.87,121.82,121.13,119.46,118.47,115.15,114.87,112.60,42.01,12.84$. MS (ESI) $\mathrm{m} / \mathrm{z}$ 
$=522.1[\mathrm{M}-\mathrm{H}]^{-}$. HRMS (ESI): calcd for $\mathrm{C}_{30} \mathrm{H}_{21} \mathrm{~N}_{3} \mathrm{O}_{5} \mathrm{~F}[\mathrm{M}-\mathrm{H}]^{-} \mathrm{m} / \mathrm{z}=522.1465$, found 522.1467. HPLC purity: $97.73 \%$.

(Z)-3-(4-((5-(4-((Cyclopropylmethyl)carbamoyl)phenyl)furan-2-yl)methylene)-3-methyl-5oxo-4,5-dihydro-1H-pyrazol-1-yl)benzoic Acid (39b)

Red solid (90 mg, 64\% yield). Isomer data: ${ }^{1} \mathrm{H}$ NMR (300 MHz, DMSO) $\delta 13.11$ (s, $1 \mathrm{H}$, COOH), 8.72-8.65 (m, 2H), $8.55(\mathrm{~d}, 1 \mathrm{H}, J=14.52 \mathrm{~Hz}), 8.19(\mathrm{~d}, 1 \mathrm{H}, J=6.15 \mathrm{~Hz})$, 8.05-7.92 (m, 4H), 7.82-7.70 (m, 2H), 7.59-7.51 (m, 2H), $3.16(\mathrm{t}, 2 \mathrm{H}, J=6.35$ and 12.28 $\left.\mathrm{Hz}, \mathrm{NHCH}_{2}\right), 2.73\left(\mathrm{~s}, 1.65 \mathrm{H}, \mathrm{CH}_{3}\right), 2.34$ (s, $\left.1.35 \mathrm{H}, \mathrm{CH}_{3}\right), 1.09-0.97$ (m, $\left.1 \mathrm{H}, \mathrm{CH}\right)$, 0.48-0.40 (m, 2H, $\left.\mathrm{CH}_{2}\right), 0.27-0.20$ (m, 2H, $\left.\mathrm{CH}_{2}\right) .{ }^{13} \mathrm{C}$ NMR (75 MHz, DMSO): $\delta 167.07$, $165.22,161.75,159.93,158.54,151.20,150.41,138.49,138.33,135.20,131.45,130.77$, $130.60,129.65,129.25,128.14,127.56,124.99,124.82,121.82,121.09,118.48,112.52$, 112.29, 43.67, 12.87, 11.02, 3.39. MS (ESI) $\mathrm{m} / \mathrm{z}=568.1[\mathrm{M}-\mathrm{H}]^{-}$. HRMS (ESI): calcd for $\mathrm{C}_{27} \mathrm{H}_{22} \mathrm{~N}_{3} \mathrm{O}_{5}[\mathrm{M}-\mathrm{H}]^{-} \mathrm{m} / \mathrm{z}=468.1559$, found 468.1561 . HPLC purity: $96.48 \%$.

(Z)-4-(4-((5-(4-((4-Fluorobenzyl)carbamoyl)phenyl)furan-2-yl)-methylene)-3-methyl-5oxo-4,5-dihydro-1H-pyrazol-1-yl)benzoic Acid (39c)

Red solid (109 mg, 77\% yield). Major $Z$-isomer data: ${ }^{1} \mathrm{H}$ NMR (300 MHz, DMSO) $\delta 12.82$ (s, $1 \mathrm{H}, \mathrm{COOH}), 9.18(\mathrm{t}, 1 \mathrm{H}, J=5.7$ and $11.64 \mathrm{~Hz}), 8.65(\mathrm{~d}, 1 \mathrm{H}, J=3.45 \mathrm{~Hz}), 8.10-7.94(\mathrm{~m}$, $8 \mathrm{H}), 7.74(\mathrm{~s}, 1 \mathrm{H}), 7.59-7.54(\mathrm{~m}, 1 \mathrm{H}), 7.40-7.32(\mathrm{~m}, 2 \mathrm{H}), 7.16(\mathrm{t}, 2 \mathrm{H}, J=8.85$ and 17.67 $\mathrm{Hz}), 4.48\left(\mathrm{~d}, 2 \mathrm{H}, J=5.55, \mathrm{NHCH}_{2}\right), 2.73$ (s, $0.64 \mathrm{H}$; minor isomer, $\left.\mathrm{CH}_{3}\right), 2.34$ (s, 2.36H; major isomer, $\left.\mathrm{CH}_{3}\right) \cdot{ }^{13} \mathrm{C}$ NMR (75 MHz, DMSO): $\delta 166.65,165.14,161.76,158.36$, $151.51,150.21,141.54,135.53,135.49,130.56,130.26,129.14,129.03,128.00,125.78$, $124.70,120.68,116.77,114.97,114.68,112.44,41.83,12.69$. MS (ESI) $\mathrm{m} / z=522.1[\mathrm{M}-$ $\mathrm{H}]^{-}$. HRMS (ESI): calcd for $\mathrm{C}_{30} \mathrm{H}_{21} \mathrm{~N}_{3} \mathrm{O}_{5} \mathrm{~F}[\mathrm{M}-\mathrm{H}]^{-} \mathrm{m} / \mathrm{z}=522.1465$, found 522.1465 . HPLC purity: $98.52 \%$.

(Z)-4-(4-((5-(4-((Cyclopropylmethyl)carbamoyl)phenyl)furan-2-yl)methylene)-3-methyl-5oxo-4,5-dihydro-1H-pyrazol-1-yl)benzoic Acid (39d).

Red solid (94 mg, 67\% yield). Major $Z$-isomer data: ${ }^{1} \mathrm{H}$ NMR (300 MHz, DMSO) $\delta 12.82$ (s, 1H, COOH), 8.70-8.64 (m, 2H,), 8.09-7.79 (m, 8H), $7.73(\mathrm{~s}, 1 \mathrm{H}), 7.57-7.53(\mathrm{~m}, 1 \mathrm{H})$, $3.15\left(\mathrm{t}, 2 \mathrm{H}, \mathrm{J}=6.0\right.$ and $\left.12.0 \mathrm{~Hz}, \mathrm{NHCH}_{2}\right), 2.72\left(\mathrm{~s}, 0.45 \mathrm{H}\right.$; minor isomer, $\left.\mathrm{CH}_{3}\right), 2.33$ (s, 2.55H; major isomer, $\left.\mathrm{CH}_{3}\right), 1.09-0.97(\mathrm{~m}, 1 \mathrm{H}, \mathrm{CH}), 0.46-0.40\left(\mathrm{~m}, 2 \mathrm{H}, \mathrm{CH}_{2}\right), 0.26-0.21(\mathrm{~m}$, $\left.2 \mathrm{H}, \mathrm{CH}_{2}\right) .{ }^{13} \mathrm{C}$ NMR (75 MHz, DMSO): $\delta 166.76,165.09,161.86,158.55,151.59,150.27$, 141.65, 135.21, 130.85, 130.44, 128.03, 125.87, 124.73, 120.71, 116.86, 112.45, 43.56, $12.79,10.90,3.27 \mathrm{MS}$ (ESI) $\mathrm{m} / z=568.1[\mathrm{M}-\mathrm{H}]^{-}$. HRMS (ESI): calcd for $\mathrm{C}_{27} \mathrm{H}_{22} \mathrm{~N}_{3} \mathrm{O}_{5}$ $[\mathrm{M}-\mathrm{H}]^{-} \mathrm{m} / \mathrm{z}=468.1559$, found 468.1557 . HPLC purity: $95.37 \%$.

\section{Overexpression and Purification of Full Length Human XPA}

The full length human XPA protein was purified according to previously described protocol. ${ }^{8,34}$ Briefly, Sf9 cells were infected with XPA virus, and the cellular pellet was lysed by dounce homogenization in buffer A containing $50 \mathrm{mM}$ Tris, $100 \mathrm{mM} \mathrm{NaCl}, 0.1 \%$ (v/v) Triton X-100, 10\% (v/v) glycerol, and $10 \mathrm{mM} \mathrm{BME,} \mathrm{along} \mathrm{with} \mathrm{a} \mathrm{protease} \mathrm{inhibitor}$ cocktail. Following sonication, imidazole was added at $1 \mathrm{mM}$ to the cellular extract, which 
was then loaded onto a $2 \mathrm{~mL}$ nickel-NTA agarose column. Bound protein was eluted in buffer A with $80 \mathrm{mM}$ imidazole, and protein containing fractions were identified using Bradford analysis. Protein containing fractions were then pooled and loaded directly onto a $2 \mathrm{~mL}$ heparin-Sepharose column. Protein was eluted using a gradient from $100 \mathrm{mM}$ to $1 \mathrm{M}$ $\mathrm{NaCl}$ in heparin buffer (50 mM Tris, pH 7.5, $1 \mathrm{mM}$ EDTA, 10\% (v/v) glycerol, and $1 \mathrm{mM}$ DTT with protease inhibitor mix). Fractions containing XPA were identified using Bradford and SDS-PAGE analysis, pooled, and dialyzed overnight in heparin buffer and stored at -80 ${ }^{\circ} \mathrm{C}$.

\section{Overexpression and Purification of $\mathrm{XPA}_{98-239}$ MBD (DBD)}

The XPA minimal binding domain was expressed in E. coli. Bl-21(DE3) from a plasmid driving expression of an $\mathrm{N}$-terminal 6-histidine tagged fragment corresponding to amino acids $98-239 .{ }^{26,42}$ The recombinant protein was purified by a two-step column chromatography procedure similar to the methodology used for full length XPA.

\section{Electrophoretic Mobility Shift Assay (EMSA)}

EMSA was carried out using previously described procedure with the following modifications. ${ }^{7,29,43}$ Reactions were performed in volume of $20 \mu \mathrm{L}$ containing a p32 labeled 30bp duplex DNA containing a single $1,2 \mathrm{dGpG}$ cisplatin adduct. ${ }^{29}$ Compounds were preincubated on ice with the indicated amount of XPA and products separated by electrophoresis on a nondenaturing $6 \%$ polyacrylamide gel. Gels were cooled and loaded, and electrophoresis was performed at $4{ }^{\circ} \mathrm{C}$. Gels were used to expose a PhosphorImager screen, band intensity was determined, and \% binding and inhibition were calculated as we have previously described. ${ }^{7,43}$

\section{DNA Intercalation Fluorescence Displacement Assay}

The analysis of DNA binding or intercalation of XPA inhibitory compounds was performed as we have described previously. ${ }^{7}$ A competitive DNA intercalation assay was performed using SYBR-Green (Sigma) and salmon sperm DNA (Fisher). Reactions were carried out in $25 \mathrm{mM}$ MOPS (pH 6.5) containing sonicated salmon sperm DNA (8.29 ng/ $\mu \mathrm{L}$ ), SYBR-

Green, and varying concentrations of XPA inhibitors. Reactions were performed in a black 96-well plate in a final volume of $110 \mu \mathrm{L}$. Doxorubicin, a known noncovalent DNA binding chemotherapeutic, was used as a positive control. Fluorescence was measured using a BioTek Synergy H1 hybrid multimode microplate reader with an excitation wavelength of $485 \mathrm{~nm}$, emission wavelength of $528 \mathrm{~nm}$, and a read height of $7 \mathrm{~mm}$. Data were collected using BioTek Gen5 reader software. Reactions were incubated a maximum of 5 min before measurements were collected.

\section{Molecular Docking}

During our previous studies ${ }^{8}$ we identified compound 1 interactions with XPA protein by examining the two solution NMR structures of the central domain of XPA (PDB codes 1XPA and 1D4U). ${ }^{24,25,44}$ Comparison of these two NMR structures showed that the putative DNA binding groove in the 1XPA structure (PDB code 1XPA) is narrower and deeper with a 
more closed conformation. The difference between these two structures may explain the flexibility of XPA in recognizing a wide variety of DNA lesions. The more closed and deep groove of 1XPA makes it more suitable for performing molecular docking experiments.

We have performed docking studies using solution NMR structure and PDB code 1XPA obtained from the Protein Data Bank (PDB) ${ }^{45}$ and prepared them using the Protein Preparation Wizard. ${ }^{46}$ In this step, force field atom types and bond orders are assigned, missing atoms are added, tautomer/ionization states are assigned, water orientations are sampled, Asn, Gln, and His residues are flipped to optimize the hydrogen bond network, and a constrained energy minimization is performed. XPA inhibitors were drawn in ChemDraw as MDL molfiles and prepared for docking using LigPrep ${ }^{47}$ including a minimization with the OPLS3 force field. ${ }^{48,49}$ All chiral centers were retained as specified in the literature. One low energy ring conformation per compound was generated. Ionization states and tautomer forms were enumerated at $\mathrm{pH} 7.0 \pm 2.0$ with Epik. ${ }^{50-52}$

XPA inhibitors were flexibly docked into the cleft defined by residues 138-142, 165-171, 174 , and $177-181$ using the Glide SP protocol ${ }^{53-55}$ with default settings. Docking poses were evaluated based on visual interrogation and calculated docking score. Potential amino acid interactions were determined based on proximity to each compound as revealed by docking analysis. XPA interactions with small molecules were viewed using Pymol using cartoon, surface, and compounds interaction views. All the molecular modeling within this study was performed using Maestro software, version 11 (Schrödinger), ${ }^{56}$ operating in a Linux environment.

\section{Supplementary Material}

Refer to Web version on PubMed Central for supplementary material.

\section{ACKNOWLEDGMENTS}

We are thankful to Dr. Lifan Zeng, Dr. Tax Georgiadis, and Jiyoon Li for technical assistance with HPLC and mass spectrometry (LCMS and HRMS). We are also grateful to Dr. Noeris K. Salam (Schrödinger LLC) for his assistance in docking studies. This work is supported by NIH Grants R01-CA180710 and R41-CA162648 and the Tom and Julie Wood Family Foundation.

\section{Abbreviations Used}

XPA

Pt

NER

HRR

MBD

DBD

EMSA xeroderma pigmentosum group A

platinum

nucleotide excision repair

homologous recombination repair

minimal binding domain

DNA binding domain

electrophoretic mobility shift assay 
SAR

Dox

RPA structure-activity relationship

doxorubicin

replication protein $\mathrm{A}$

\section{REFERENCES}

(1). Gavande NS; VanderVere-Carozza PS; Hinshaw HD; Jalal SI; Sears CR; Pawelczak KS; Turchi JJ DNA repair targeted therapy: The past or future of cancer treatment? Pharmacol. Ther. 2016, 160, 65-83. [PubMed: 26896565]

(2). Wang Y-Q; Wang P-Y; Wang Y-T; Yang G-F; Zhang A; Miao Z-H An update on poly(ADPribose)polymerase-1 (PARP-1) inhibitors: Opportunities and challenges in cancer therapy. J. Med. Chem. 2016, 59, 9575-9598. [PubMed: 27416328]

(3). Lee J-M; Ledermann JA; Kohn EC PARP Inhibitors for BRCA $1 \frac{1}{2}$ mutation-associated and BRCAlike malignancies. Ann. Oncol 2014, 25, 32-40. [PubMed: 24225019]

(4). Galluzzi L; Senovilla L; Vitale I; Michels J; Martins I; Kepp O; Castedo M; Kroemer G Molecular mechanisms of cisplatin resistance. Oncogene 2012, 31, 1869-1883. [PubMed: 21892204]

(5). Martin LP; Hamilton TC; Schilder RJ Platinum resistance: The role of DNA repair pathways. Clin. Cancer Res. 2008, 14, 1291-1295. [PubMed: 18316546]

(6). Gavande NS; VanderVere-Carozza PS; Pawelczak KS; Turchi JJ Targeting the nucleotide excision repair pathway for therapeutic applications In DNA Repair in Cancer Therapy: Molecular Targets and Clinical Applications; Kelley M, Ed.; Elsevier Academic Press: Amsterdam, The Netherlands, 2016; pp 135-150.

(7). Mishra AK; Dormi SS; Turchi AM; Woods DS; Turchi JJ Chemical inhibitor targeting the Replication Protein A-DNA interaction increases the efficacy of Pt-based chemotherapy in lung and ovarian cancer. Biochem. Pharmacol. 2015, 93, 25-33. [PubMed: 25449597]

(8). Neher TM; Shuck SC; Liu JY; Zhang JT; Turchi JJ Identification of novel small molecule inhibitors of the XPA protein using in silico based screening. ACS Chem. Biol. 2010, 5, 953965. [PubMed: 20662484]

(9). Fu X; Hu J; Han HY; Hua YJ; Zhou L; Shuai WD; Du WY; Kuang CM; Chen S; Huang W; Liu RY High expression of XPA confers poor prognosis for nasopharyngeal carcinoma patients treated with platinum-based chemoradiotherapy. Oncotarget 2015, 6, 28478-28490. [PubMed: 26156020]

(10). Liu Y; Bernauer AM; Yingling CM; Belinsky SA HIF1 regulated expression of XPA contributes to cisplatin resistance in lung cancer. Carcinogenesis 2012, 33, 1187-1192. [PubMed: 22467238]

(11). Weaver DA; Crawford EL; Warner KA; Elkhairi F; Khuder SA; Willey JC ABCC5, ERCC2, XPA and XRCC1 transcript abundance levels correlate with cisplatin chemoresistance in nonsmall cell lung cancer cell lines. Mol. Cancer 2005, 4, 18. [PubMed: 15882455]

(12). Lai TC; Chow KC; Fang HY; Cho HC; Chen CY; Lin TY; Chiang IP; Ho SP Expression of xeroderma pigmentosum complementation group $\mathrm{C}$ protein predicts cisplatin resistance in lung adenocarcinoma patients. Oncol. Rep. 2011, 25, 1243-1251. [PubMed: 21327329]

(13). Jun HJ; Ahn MJ; Kim HS; Yi SY; Han J; Lee SK; Ahn YC; Jeong HS; Son YI; Baek JH; Park K ERCC1 expression as a predictive marker of squamous cell carcinoma of the head and neck treated with cisplatin-based concurrent chemoradiation. Br. J. Cancer 2008, 99, 167-172. [PubMed: 18594541]

(14). Jekimovs C; Bolderson E; Suraweera A; Adams M; O’Byrne KJ; Richard DJ Chemotherapeutic compounds targeting the DNA double-strand break repair pathways: the good, the bad, and the promising. Front. Oncol 2014, 4, 86. [PubMed: 24795863]

(15). Luijsterburg MS; von Bornstaedt G; Gourdin AM; Politi AZ; Mone MJ; Warmerdam DO; Goedhart J; Vermeulen W; van Driel R; Hofer T Stochastic and reversible assembly of a multiprotein DNA repair complex ensures accurate target site recognition and efficient repair. J. Cell Biol. 2010, 189, 445-463. [PubMed: 20439997] 
(16). Koberle B; Roginskaya V; Wood RD XPA protein as a limiting factor for nucleotide excision repair and UV sensitivity in human cells. DNA Repair 2006, 5, 641-648. [PubMed: 16413230]

(17). Sugitani N; Sivley RM; Perry KE; Capra JA; Chazin WJ XPA: A key scaffold for human nucleotide excision repair. DNA Repair 2016, 44, 123-135. [PubMed: 27247238]

(18). Fadda E Role of the XPA protein in the NER pathway: A perspective on the function of structural disorder in macromolecular assembly. Comput. Struct. Biotechnol. J 2016, 14, 78-85. [PubMed: 26865925]

(19). Welsh C; Day R; McGurk C; Masters JRW; Wood RD; Koberle B Reduced levels of XPA, ERCC1 and XPF DNA repair proteins in testis tumor cell lines. Int. J. Cancer 2004, 110, 352361. [PubMed: 15095299]

(20). Einhorn LH Curing metastatic testicular cancer. Proc. Natl. Acad. Sci. U. S. A. 2002, 99, 45924595. [PubMed: 11904381]

(21). Kaelin WG Jr. The concept of synthetic lethality in the context of anticancer therapy. Nat. Rev. Cancer 2005, 5, 689-698. [PubMed: 16110319]

(22). Chan DA; Giaccia AJ Harnessing synthetic lethal interactions in anticancer drug discovery. Nat. Rev. Drug Discovery 2011, 10, 351-364. [PubMed: 21532565]

(23). Ashworth AA Synthetic lethal therapeutic approach: poly(ADP) ribose polymerase inhibitors for the treatment of cancers deficient in DNA double-strand break repair. J. Clin. Oncol. 2008, 26, 3785-3790. [PubMed: 18591545]

(24). Ikegami T; Kuraoka I; Saijo M; et al. Solution structure of the DNA- and RPA-binding domain of the human repair factor XPA. Nat. Struct. Mol. Biol. 1998, 5, 701-706.

(25). Buchko GW; Ni S; Thrall BD; Kennedy MA Structural features of the minimal DNA binding domain (M98-F219) of human nucleotide excision repair protein XPA. Nucleic Acids Res. 1998, 26, 2779-2788. [PubMed: 9592168]

(26). Sugitani N; Shell SM; Soss SE; Chazin WJ Redefining the DNA-binding domain of human XPA. J. Am. Chem. Soc. 2014, 136, 10830-10833. [PubMed: 25056193]

(27). Hilton B; Shkriabai N; Musich PR; Kvaratskhelia M; Shell S; Zou Y A new structural insight into XPA-DNA interactions. Biosci. Rep. 2014, 34, 831-840.

(28). Saijo M; Takedachi A; Tanaka K Nucleotide excision repair by mutant Xeroderma Pigmentosum Group A (XPA) proteins with deficiency in interaction with RPA. J. Biol. Chem. 2011, 286, 5476-5483. [PubMed: 21148310]

(29). Patrick SM; Turchi JJ Xeroderma pigmentosum complementation group A protein (XPA) modulates RPA-DNA interactions via enhanced complex stability and inhibition of strand separation activity. J. Biol. Chem. 2002, 277, 16096-16101. [PubMed: 11859086]

(30). Koch SC; Kuper J; Gasteiger KL; Simon N; Strasser R; Eisen D; Geiger S; Schneider S; Kisker C; Carell T Structural insights into the recognition of cisplatin and AAF-dG lesion by Rad14 (XPA). Proc. Natl. Acad. Sci. U. S. A. 2015, 112, 8272-8277. [PubMed: 26100901]

(31). Yang Z; Roginskaya M; Colis LC; Basu AK; Shell SM; Liu Y; Musich PR; Harris CM; Harris TM; Zou Y Specific and efficient binding of xeroderma pigmentosum complementation group A to double-strand/single-strand DNA junctions with $3^{\prime}$ - and/or $5^{\prime}$-ssDNA branches. Biochemistry 2006, 45, 15921-15930. [PubMed: 17176115]

(32). Buchko GW; Tung CS; McAteer K; Isern NG; Spicer LD; Kennedy MA DNA-XPA interactions: a (31)P NMR and molecular modeling study of dCCAATAACC association with the minimal DNA-binding domain (M98-F219) of the nucleotide excision repair protein XPA. Nucleic Acids Res. 2001, 29, 2635-2643. [PubMed: 11410673]

(33). Camenisch U; Dip R; Schumacher SB; Schuler B; Naegeli H Recognition of helical kinks by xeroderma pigmentosum group A protein triggers DNA excision repair. Nat. Struct. Mol. Biol. 2006, 13, 278-284. [PubMed: 16491090]

(34). Hermanson IL; Turchi JJ Overexpression and purification of human XPA using a Baculovirus expression system. Protein Expression Purif. 2000, 19, 1-11.

(35). Hellman LM; Fried MG Electrophoretic mobility shift assay (EMSA) for detecting proteinnucleic acid interactions. Nat. Protoc. 2007, 2, 1849-1861. [PubMed: 17703195] 
(36). Kumar V; Chang C-K; Tan K-P; Jung Y-S; Chen S-H; Cheng Y-SE; Liang P-H Identification, synthesis, and evaluation of new neuraminidase inhibitors. Org. Lett. 2014, 16, 5060-5063. [PubMed: 25229881]

(37). Liang Z; Ding X; Ai J; Kong X; Chen L; Chen L; Luo C; Geng M; Liu H; Chen K; Jiang H Discovering potent inhibitors against c-Met kinase: molecular design, organic synthesis and bioassay. Org. Biomol. Chem. 2012, 10, 421-430. [PubMed: 22108637]

(38). Bowers EM; Yan G; Mukherjee C; et al. Virtual ligand screening of the p300/CBP histone acetyltransferase: Identification of a selective small molecule inhibitor. Chem. Biol. 2010, 17, 471-482. [PubMed: 20534345]

(39). Moreau F; Desroy N; Genevard JM; et al. Discovery of new gram-negative antivirulence drugs: Structure and properties of novel E. coli WaaC inhibitors. Bioorg. Med. Chem. Lett. 2008, 18, 4022-4026. [PubMed: 18571407]

(40). Wang M; Mahrenholz A; Lee S-H RPA stabilizes the XPA-damaged DNA complex through protein-protein interaction. Biochemistry 2000, 39, 6433-6439. [PubMed: 10828957]

(41). Lee S-H; Kim DK; Drissi R Human Xeroderma Pigmentosum Group A protein interacts with human Replication Protein A and inhibits DNA replication. J. Biol. Chem. 1995, 270, 2180021805. [PubMed: 7665601]

(42). Jones CJ; Wood RD Preferential binding of the Xeroderma Pigmentosum Group A complementing protein to damaged DNA. Biochemistry 1993, 32, 12096-12104. [PubMed: 8218288]

(43). Shuck SC; Turchi JJ Targeted inhibition of Replication Protein A reveals cytotoxic activity, synergy with chemotherapeutic DNA-damaging agents, and insight into cellular function. Cancer Res. 2010, 70, 3189-3198. [PubMed: 20395205]

(44). Buchko GW; Daughdrill GW; de Lorimier R; Rao BK; Isern NG; Lingbeck JM; Taylor J-S; Wold MS; Gochin M; Spicer LD; Lowry DF; Kennedy MA Interactions of human nucleotide excision repair protein XPA with DNA and RPA70 Delta C327: chemical shift mapping and 15N NMR relaxation studies. Biochemistry 1999, 38, 15116-15128. [PubMed: 10563794]

(45). Berman HM; Westbrook J; Feng Z; Gilliland G; Bhat TN; Weissig H; Shindyalov IN; Bourne PE The Protein Data Bank. Nucleic Acids Res. 2000, 28, 235-242. [PubMed: 10592235]

(46). Sastry GM; Adzhigirey M; Day T; Annabhimoju R; Sherman W Protein and ligand preparation: Parameters, protocols, and influence on virtual screening enrichments. J. Comput.-Aided Mol. Des 2013, 27, 221-234. [PubMed: 23579614]

(47). Schrodinger release 2015-2: LigPrep, version 3.4; Schrödinger LLC: New York, NY, 2015.

(48). Shivakumar D; Harder E; Damm W; Friesner RA; Sherman W Improving the prediction of absolute solvation free energies using the next generation OPLS force field. J. Chem. Theory Comput. 2012, 8, 2553-8. [PubMed: 26592101]

(49). Harder E; Damm W; Maple J; Wu C; Reboul M; Xiang JY; Wang L; Lupyan D; Dahlgren MK; Knight JL; Kaus JW; Cerutti DS; Krilov G; Jorgensen WL; Abel R; Friesner RA OPLS3: A force field providing broad coverage of drug-like small molecules and proteins. J. Chem. Theory Comput. 2016, 12, 281-296. [PubMed: 26584231]

(50). Shelley JC; Cholleti A; Frye LL; Greenwood JR; Timlin MR; Uchimaya M Epik: A software program for $\mathrm{Pk}$ (a) prediction and protonation state generation for drug-like molecules. J. Comput.-Aided Mol. Des. 2007, 21, 681-91.

(51). Greenwood JR; Calkins D; Sullivan AP; Shelley JC Towards the comprehensive, rapid, and accurate prediction of the favorable tautomeric states of drug-like molecules in aqueous solution. J. Comput.-Aided Mol. Des. 2010, 24, 591-604.

(52). Schrodinger release 2015-2: Epik, version 3.2; Schrödinger LLC: New York, NY, 2015.

(53). Friesner RA; Banks JL; Murphy RB; Halgren TA; Klicic JJ; Mainz DT; Repasky MP; Knoll EH; Shelley M; Perry JK; Shaw DE; Francis P; Shenkin PS Glide: A new approach for rapid, accurate docking and scoring. 1. Method and assessment of docking accuracy. J. Med. Chem. 2004, 47, 1739-49. [PubMed: 15027865]

(54). Halgren TA; Murphy RB; Friesner RA; Beard HS; Frye LL; Pollard WT; Banks JL Glide: A new approach for rapid, accurate docking and scoring. 2. Enrichment factors in database screening. J. Med. Chem. 2004, 47, 1750-59. [PubMed: 15027866] 
(55). Small-molecule drug discovery suite 2015-2: Glide, version 6.7; Schrödinger LLC: New York, NY, 2015.

(56). Maestro, version 11; Schrödinger, LLC, New York, NY, 2017. 


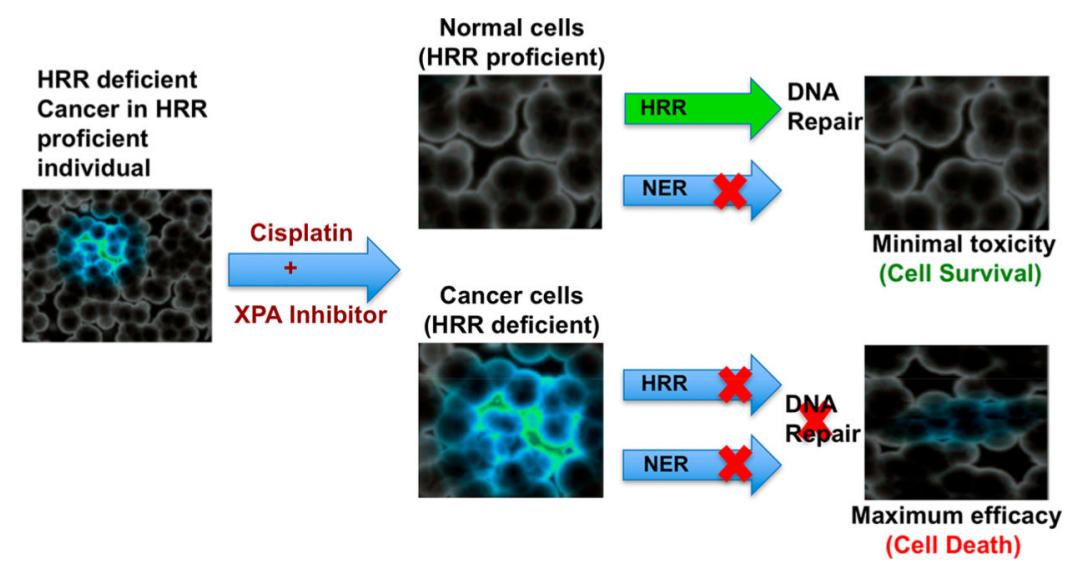

\section{Figure 1.}

Chemical synthetic lethality in Pt-combination cancer therapy. A HRR (homologous recombination repair) deficient cancer (blue cells) is depicted in a HRR proficient patient (black cells). Treatment with an XPA inhibitor (or NER inhibitor) renders both cell types unable to repair the Pt-DNA adducts via NER. The HRR proficient normal cells are able to tolerate or repair the Pt-DNA damage, which is anticipated to reduce toxicity. The HRR deficient cancer cells are unable to repair or tolerate the Pt-damage and thus are sensitive to the combination therapy. 


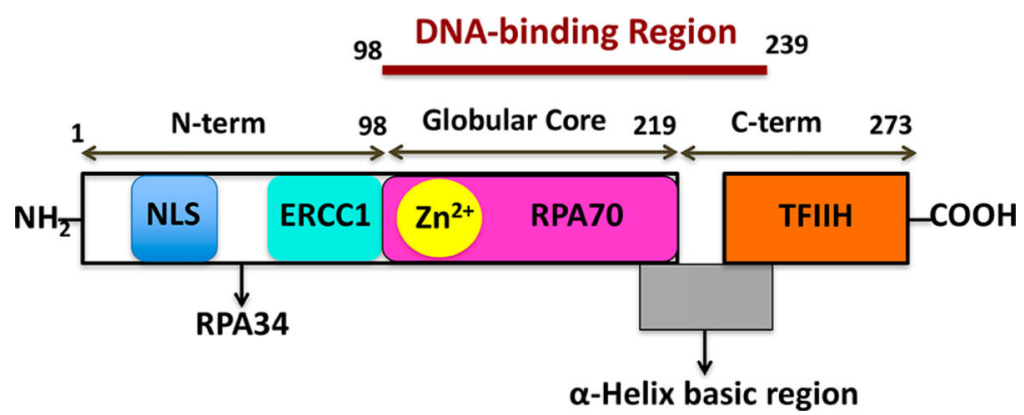

Figure 2.

Schematic representation of human XPA protein and XPA interaction partners, mainly NER proteins. 
A

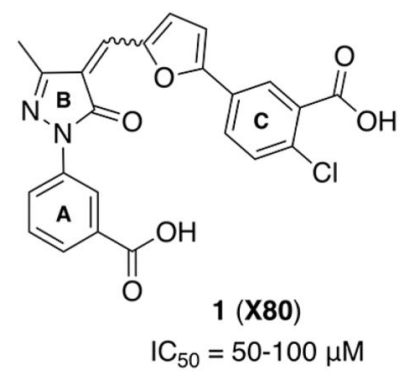

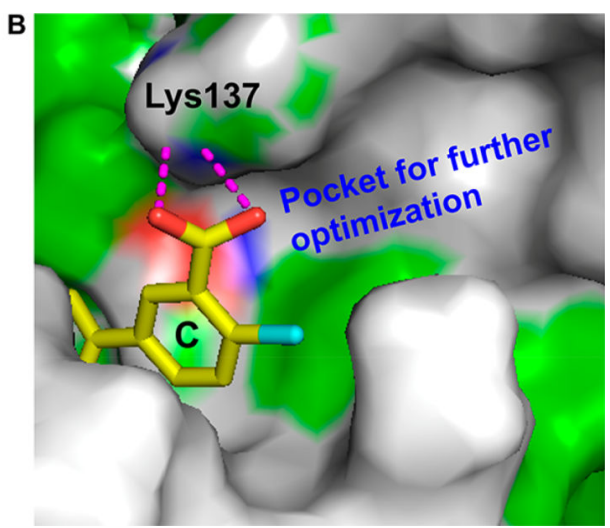

Figure 3.

(A) Structure of compound 1. (B) Schematic representation of SAR exploration rationale: a pocket surrounding ring $\mathrm{C}$ of compound $\mathbf{1}$ for further structural optimization. Potential hydrogen bond contacts are shown as dashed magenta lines. 


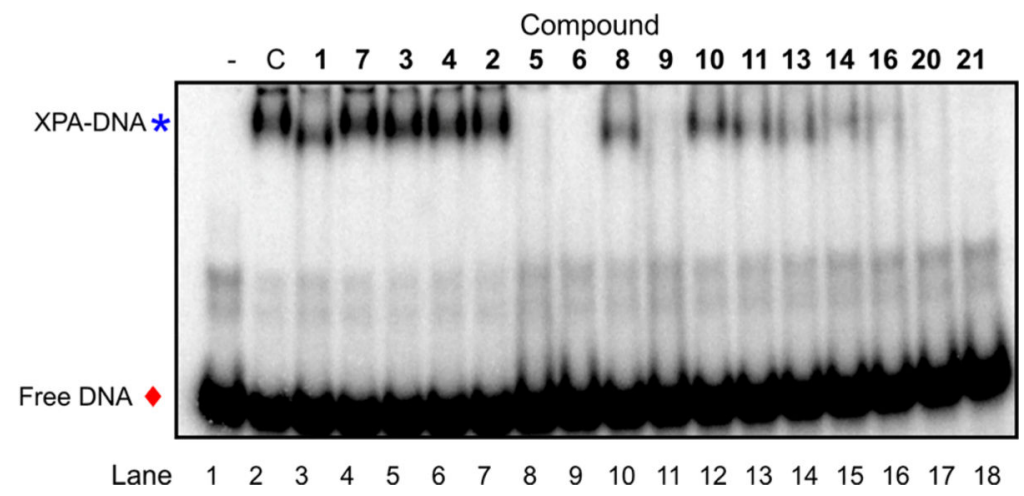

Figure 4.

EMSA analysis of XPA-DNA binding: Purified XPA (lanes 2-18) was mixed with DMSO control (lane $2, \mathrm{C}$ ) or the indicated compound at a concentration of $50 \mu \mathrm{M}$ (lanes $3-18$ ). ${ }^{32} \mathrm{P}$ labeled ds-platinum damaged DNA was added and incubation continued for $30 \mathrm{~min}$ on ice. The products were separated on a $6 \%$ native gel by electrophoresis at $4{ }^{\circ} \mathrm{C}$. Products were detected and quantified by PhosphorImager analysis. The free DNA is indicated by the red diamond and the XPA-DNA complex by the blue asterisk. 

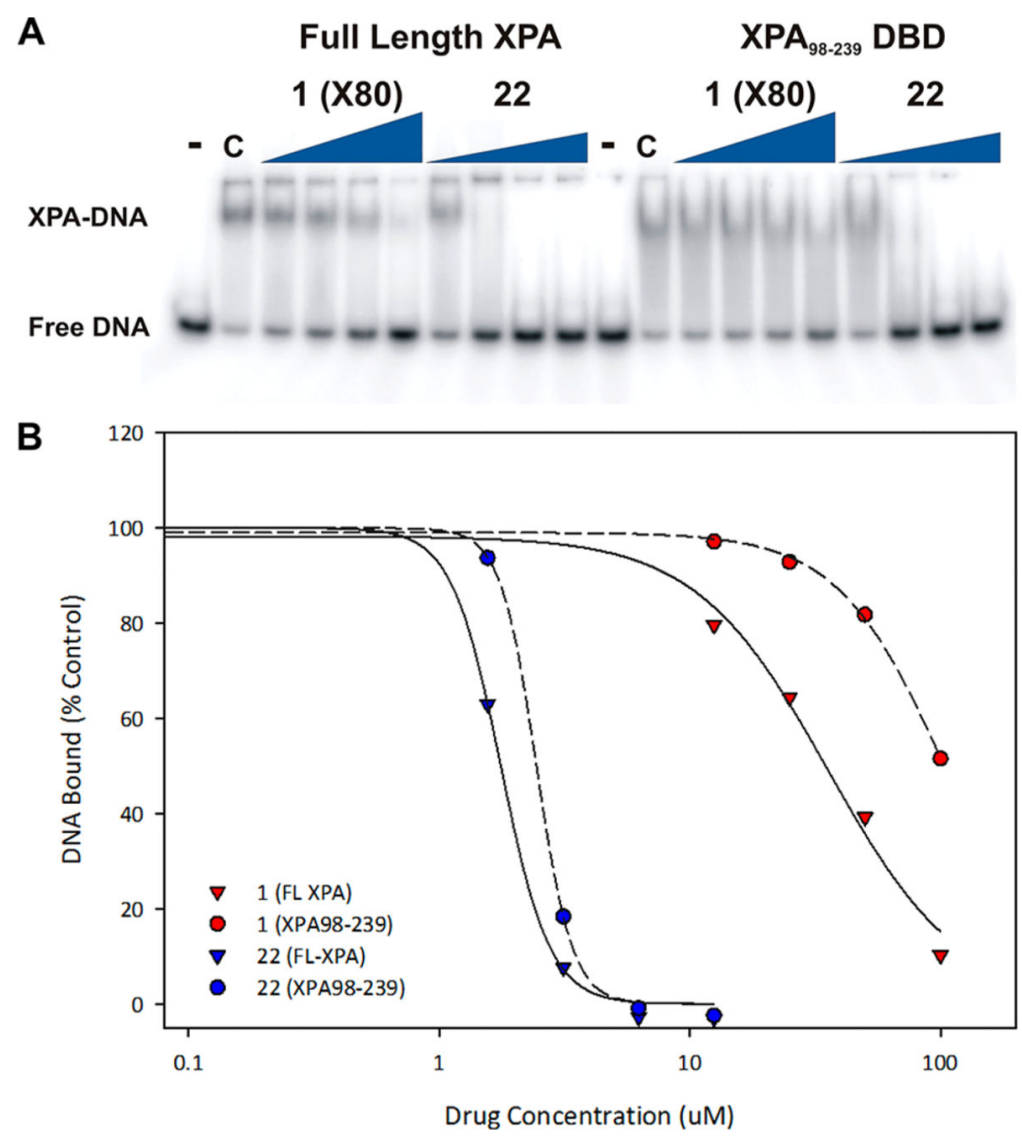

Figure 5.

(A) EMSA with increasing concentration of compound $1(12.5-100 \mu \mathrm{M})$ and $22(1.6-25$ $\mu \mathrm{M}$ ) relative to DMSO control on full-length XPA (FL XPA) and XPA98-239 DBD. (B) Quantification and concentration-dependent analysis of DNA-binding activity of compounds 1 and 22 relative to DMSO control on full-length XPA and XPA98-239 DBD. 

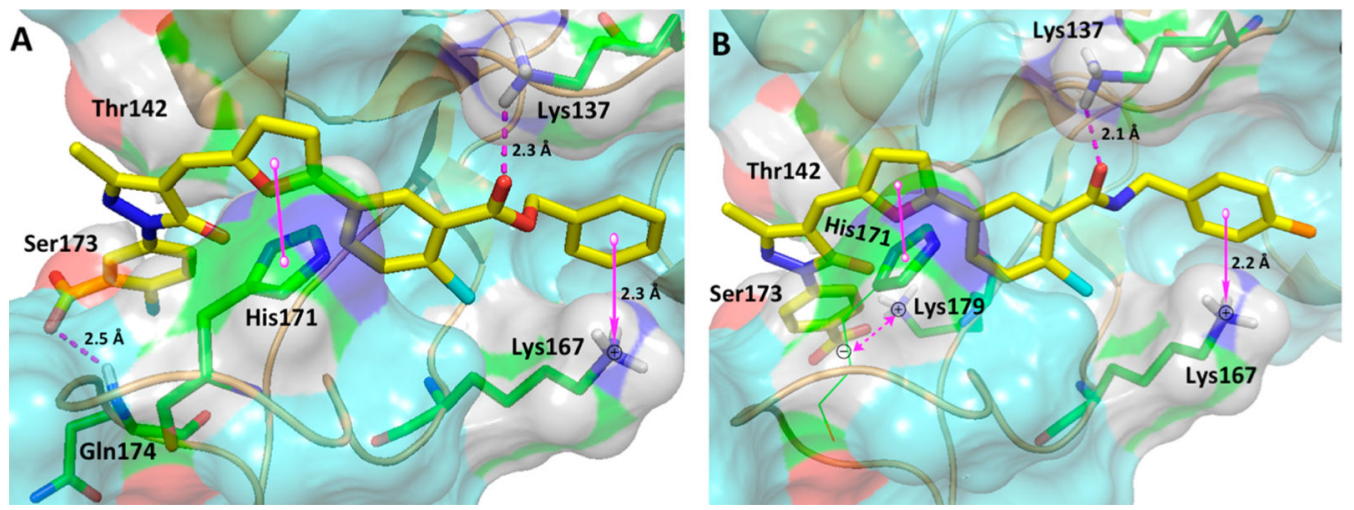

Figure 6.

Molecular docking studies (PDB code 1XPA). (A) Molecular interactions of compound 22 (yellow carbon) $Z$-isomer with hXPA (key amino acids are shown in green carbon, surface is shown in aquamarine, and cartoon is shown in light orange). (B) Molecular interactions of 34i (yellow carbon) $Z$-isomer with hXPA (key amino acids are shown in green carbon, surface is shown in aquamarine, and cartoon is shown in light orange). Interaction with amino acid side chains is indicated with the dashed magenta lines, $\pi-\pi$ stacking interactions are shown in solid magenta dumbbell, cation $-\pi$ interactions are shown in solid one sided magenta arrow, and salt-bridge interactions are shown in dashed two-sided magenta arrow. Distances are indicated in $\AA$. 


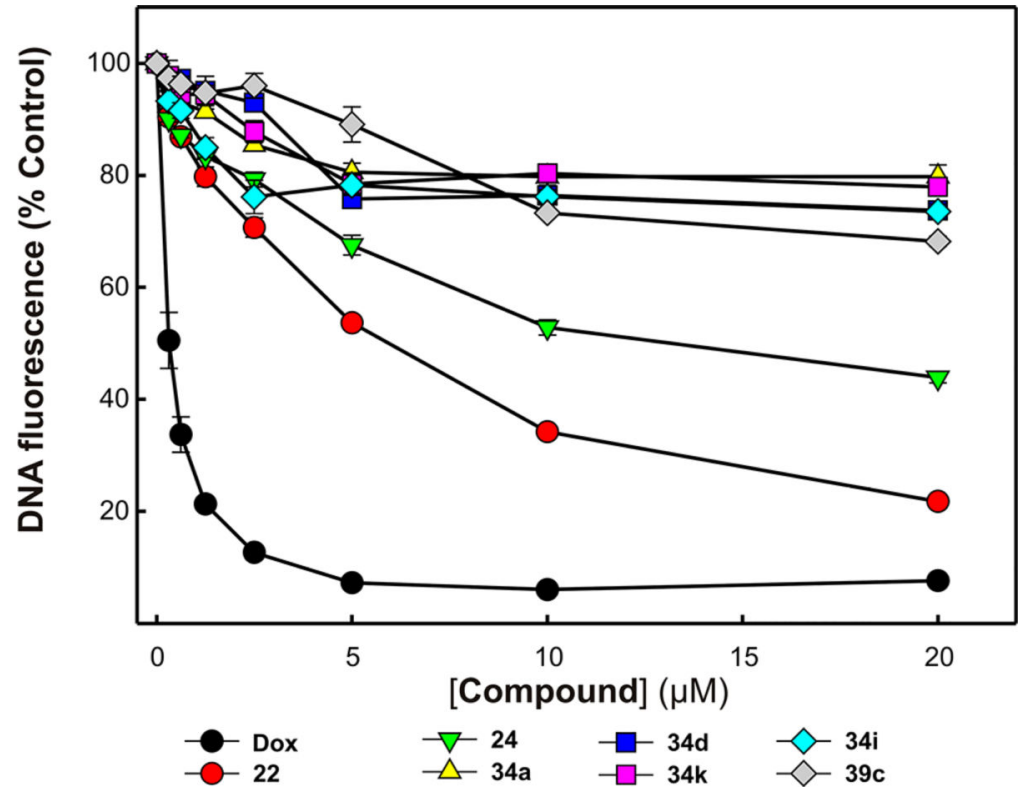

Figure 7.

Analysis of compound interactions with DNA. The indicated concentrations of compounds 22, 24, 34a, 34d, 34i, 34k, and 39c were analyzed for the ability to displace a fluorescent Sybr-green DNA intercalator as a measure of compound DNA interactions. The assay was performed and fluorescence measured as described in "Experimental Details". The data represent the average and SD of three independent experimental determinations performed in duplicates. 

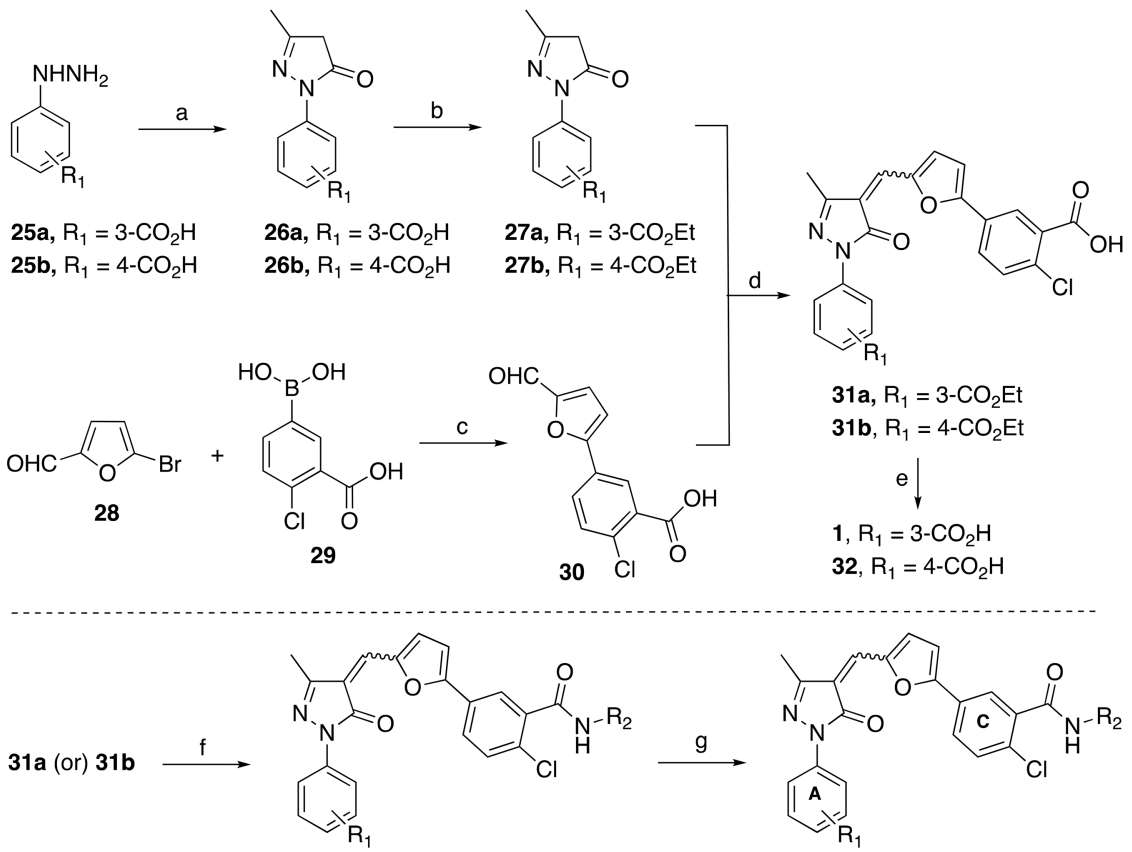

33a, $\mathrm{R}_{1}=3-\mathrm{CO}_{2} \mathrm{Et} ; \mathrm{R}_{2}=4$-fluorobenzyl

34a, $\mathrm{R}_{1}=3-\mathrm{CO}_{2} \mathrm{H} ; \mathrm{R}_{2}=4$-fluorobenzyl

33b, $\mathrm{R}_{1}=3-\mathrm{CO}_{2} \mathrm{Et} ; \mathrm{R}_{2}=$ 3-methoxyphenyl

34b, $\mathrm{R}_{1}=3-\mathrm{CO}_{2} \mathrm{H} ; \mathrm{R}_{2}=3$-methoxyphenyl

33c, $\mathrm{R}_{1}=3-\mathrm{CO}_{2} \mathrm{Et} ; \mathrm{R}_{2}=3$,4-dimethoxyphenyl

33d, $\mathrm{R}_{1}=3-\mathrm{CO}_{2} \mathrm{Et} ; \mathrm{R}_{2}=$ cyclopropylmethy

33e, $\mathrm{R}_{1}=3-\mathrm{CO}_{2} \mathrm{Et} ; \mathrm{R}_{2}=$ cyclopropyl

33f, $\mathrm{R}_{1}=3-\mathrm{CO}_{2} \mathrm{Et} ; \mathrm{R}_{2}=$ 4-tetrahydropyran

34c, $\mathrm{R}_{1}=3-\mathrm{CO}_{2} \mathrm{H} ; \mathrm{R}_{2}=3,4$-dimethoxyphenyl

34d, $\mathrm{R}_{1}=3-\mathrm{CO}_{2} \mathrm{H} ; \mathrm{R}_{2}=$ cyclopropylmethyl

34e, $\mathrm{R}_{1}=3-\mathrm{CO}_{2} \mathrm{NH}$-cyclopropylmethyl; $\mathrm{f}$

$\mathrm{R}_{2}=$ cyclopropylmethyl

33g, $\mathrm{R}_{1}=3-\mathrm{CO}_{2} \mathrm{Et}$;

$\mathrm{R}_{2}=$ 4-methyltetrahydropyran

34f, $\mathrm{R}_{1}=3-\mathrm{CO}_{2} \mathrm{H} ; \mathrm{R}_{2}=$ cyclopropyl

34g, $\mathrm{R}_{1}=3-\mathrm{CO}_{2} \mathrm{H} ; \mathrm{R}_{2}=$ 4-tetrahydropyran

33h, $\mathrm{R}_{1}=4-\mathrm{CO}_{2} \mathrm{Et} ; \mathrm{R}_{2}=4$-fluorobenzyl

33i, $\mathrm{R}_{1}=4-\mathrm{CO}_{2} \mathrm{Et} ; \mathrm{R}_{2}=3$-methoxyphenyl

34h, $\mathrm{R}_{1}=3-\mathrm{CO}_{2} \mathrm{H} ; \mathrm{R}_{2}=4$-methyltetrahydropyran

$34 \mathrm{i}, \mathrm{R}_{1}=4-\mathrm{CO}_{2} \mathrm{H} ; \mathrm{R}_{2}=4$-fluorobenzyl

33j, $\mathrm{R}_{1}=4-\mathrm{CO}_{2} \mathrm{Et} ; \mathrm{R}_{2}=$ cyclopropylmethyl

34j, $\mathrm{R}_{1}=4-\mathrm{CO}_{2} \mathrm{H} ; \mathrm{R}_{2}=3$-methoxyphenyl

34k, $\mathrm{R}_{1}=4-\mathrm{CO}_{2} \mathrm{H} ; \mathrm{R}_{2}=$ cyclopropylmethyl

\section{Scheme 1. Synthesis of Analogs 34a-k}

${ }^{a}$ Reagents and conditions: (a) ethyl acetoacetate, AcOH, reflux for 12 h, 72-76\%; (b)

$\mathrm{H}_{2} \mathrm{SO}_{4}$, EtOH, reflux for $12 \mathrm{~h}, 77-80 \%$; (c) $\mathrm{Pd}\left(\mathrm{PPh}_{3}\right)_{4}, \mathrm{~K}_{2} \mathrm{CO}_{3}$, toluene/EtOH/H $\mathrm{H}_{2} \mathrm{O}$ (1:1:0.3), $90{ }^{\circ} \mathrm{C}$ for $15 \mathrm{~h}, 92 \%$; (d) $\mathrm{AcOH}$, reflux for $3 \mathrm{~h}, 76-87 \%$; (e) $2 \mathrm{~N} \mathrm{NaOH}, \mathrm{THF} /$ $\mathrm{MeOH}(2: 1)$, rt for $6 \mathrm{~h}, 82-85 \%$; (f) alkyl- or arylamine, EDCI, HOBt, DIPEA, DMF, rt for 18 h, 62-81\%; (g) LiOH, THF/EtOH/ $\mathrm{H}_{2} \mathrm{O}$ (4:2:1), rt for 12 h, 60-76\% (after recrystallization). 


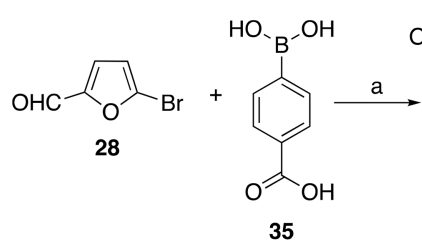

$\mathrm{OHC}$<smiles>O=C(O)c1ccc(-c2ccc(I)o2)cc1</smiles>

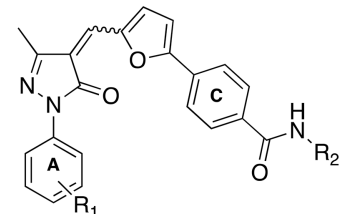

39a, $\mathrm{R}_{1}=3-\mathrm{CO}_{2} \mathrm{H} ; \mathrm{R}_{2}=4$-fluorobenzyl 39b, $\mathrm{R}_{1}=3-\mathrm{CO}_{2} \mathrm{H} ; \mathrm{R}_{2}=$ cyclopropylmethyl $39 \mathrm{c}, \mathrm{R}_{1}=4-\mathrm{CO}_{2} \mathrm{H} ; \mathrm{R}_{2}=4$-fluorobenzyl 39d, $\mathrm{R}_{1}=4-\mathrm{CO}_{2} \mathrm{H} ; \mathrm{R}_{2}=$ cyclopropylmethyl

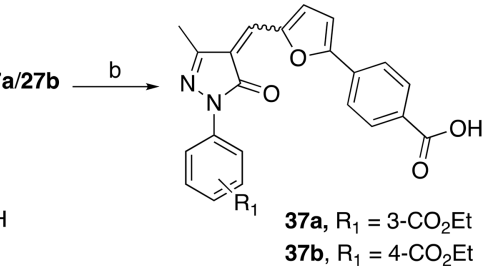

c|

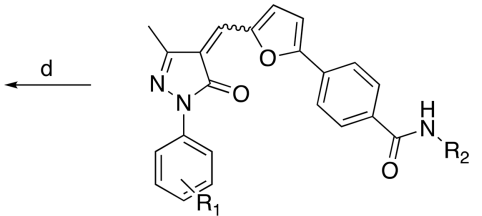

38a, $\mathrm{R}_{1}=3-\mathrm{CO}_{2} \mathrm{Et} ; \mathrm{R}_{2}=4$-fluorobenzyl $38 \mathrm{~b}, \mathrm{R}_{1}=3-\mathrm{CO}_{2} \mathrm{Et} ; \mathrm{R}_{2}=$ cyclopropylmethyl 38c, $\mathrm{R}_{1}=4-\mathrm{CO}_{2} \mathrm{Et} ; \mathrm{R}_{2}=4$-fluorobenzyl

38d, $R_{1}=4-\mathrm{CO}_{2} E t ; R_{2}=$ cyclopropylmethyl

Scheme 2. Synthesis of Analogs 39a-d $a$

a Reagents and conditions: (a) $\mathrm{Pd}\left(\mathrm{PPh}_{3}\right)_{4}, \mathrm{~K}_{2} \mathrm{CO}_{3}$, toluene/EtOH/ $\mathrm{H}_{2} \mathrm{O}(1: 1: 0.3), 90{ }^{\circ} \mathrm{C}$ for 15 h, 90\%; (b) AcOH, reflux for 3 h, 78-85\%; (c) alkylor arylamine, EDCI, HOBt, DIPEA, DMF, rt for 18 h, 74-80\%; (d) LiOH, THF/EtOH/ $\mathrm{H}_{2} \mathrm{O}(4: 2: 1)$, rt for $12 \mathrm{~h}, 64-77 \%$ (after recrystallization). 


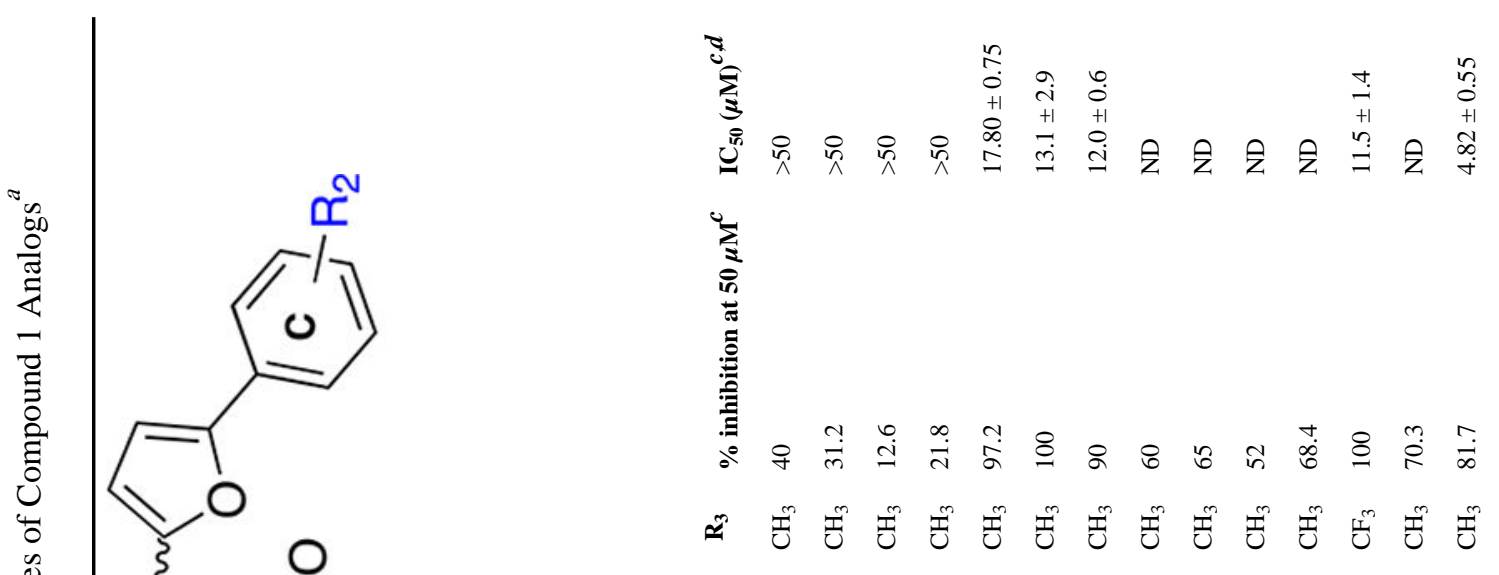




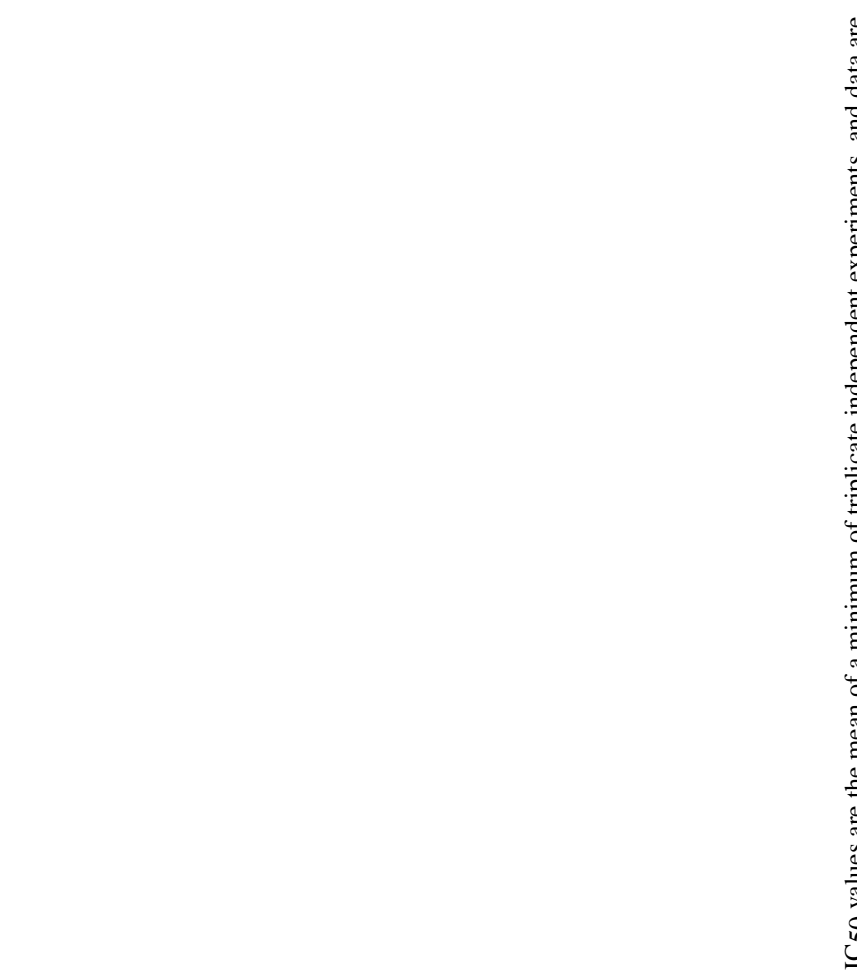

로을
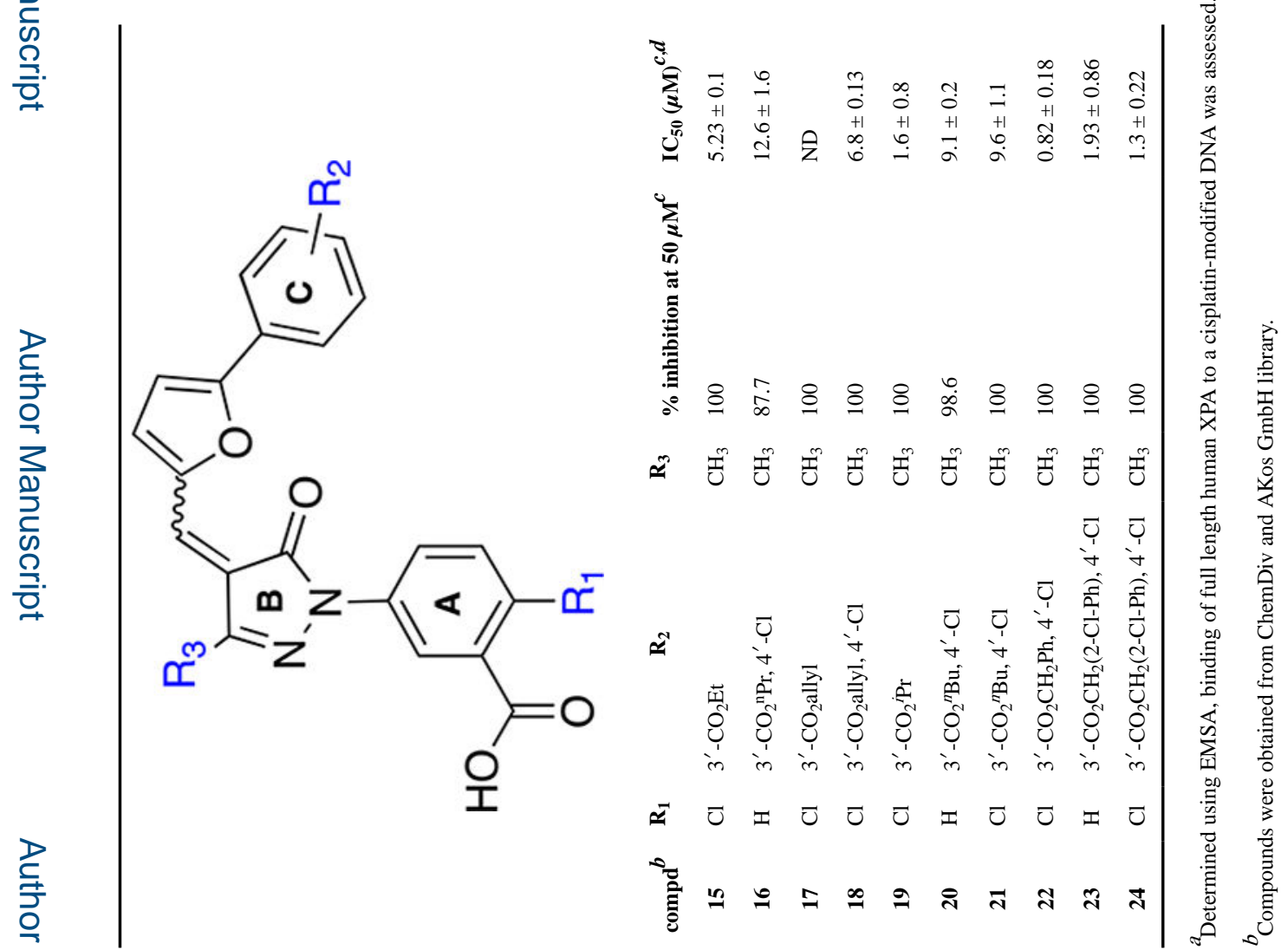

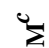
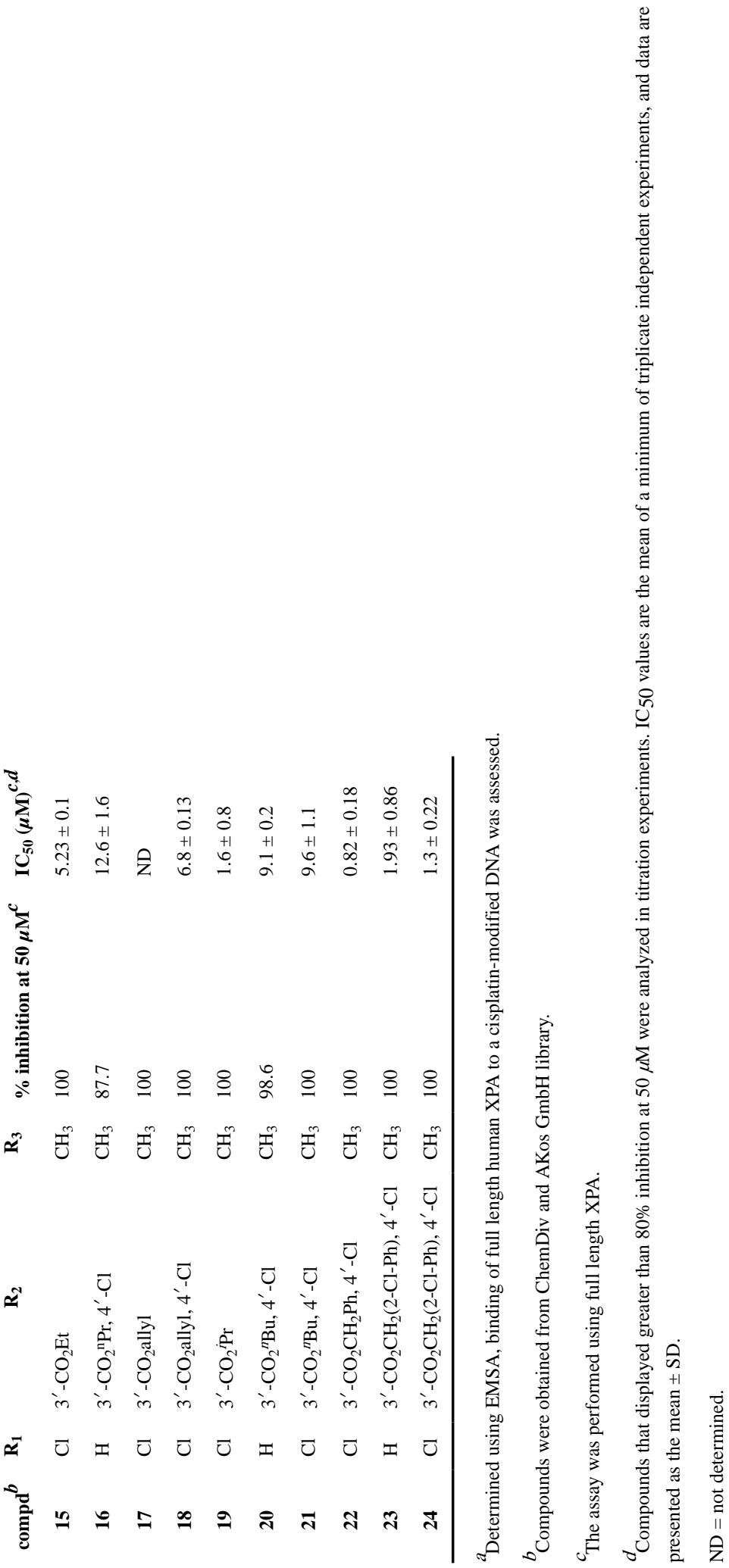

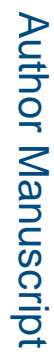

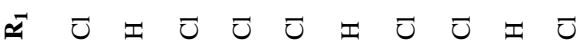

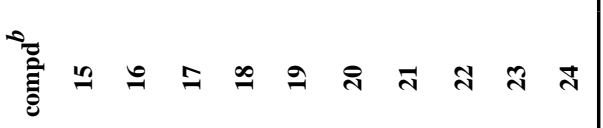

^ ปี

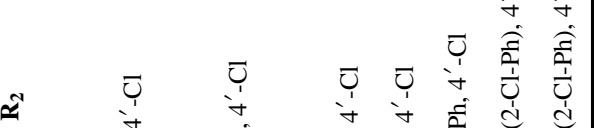

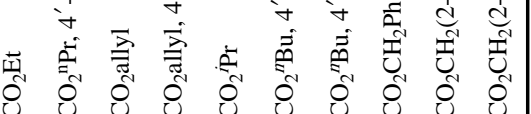

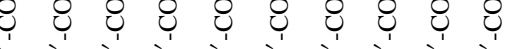



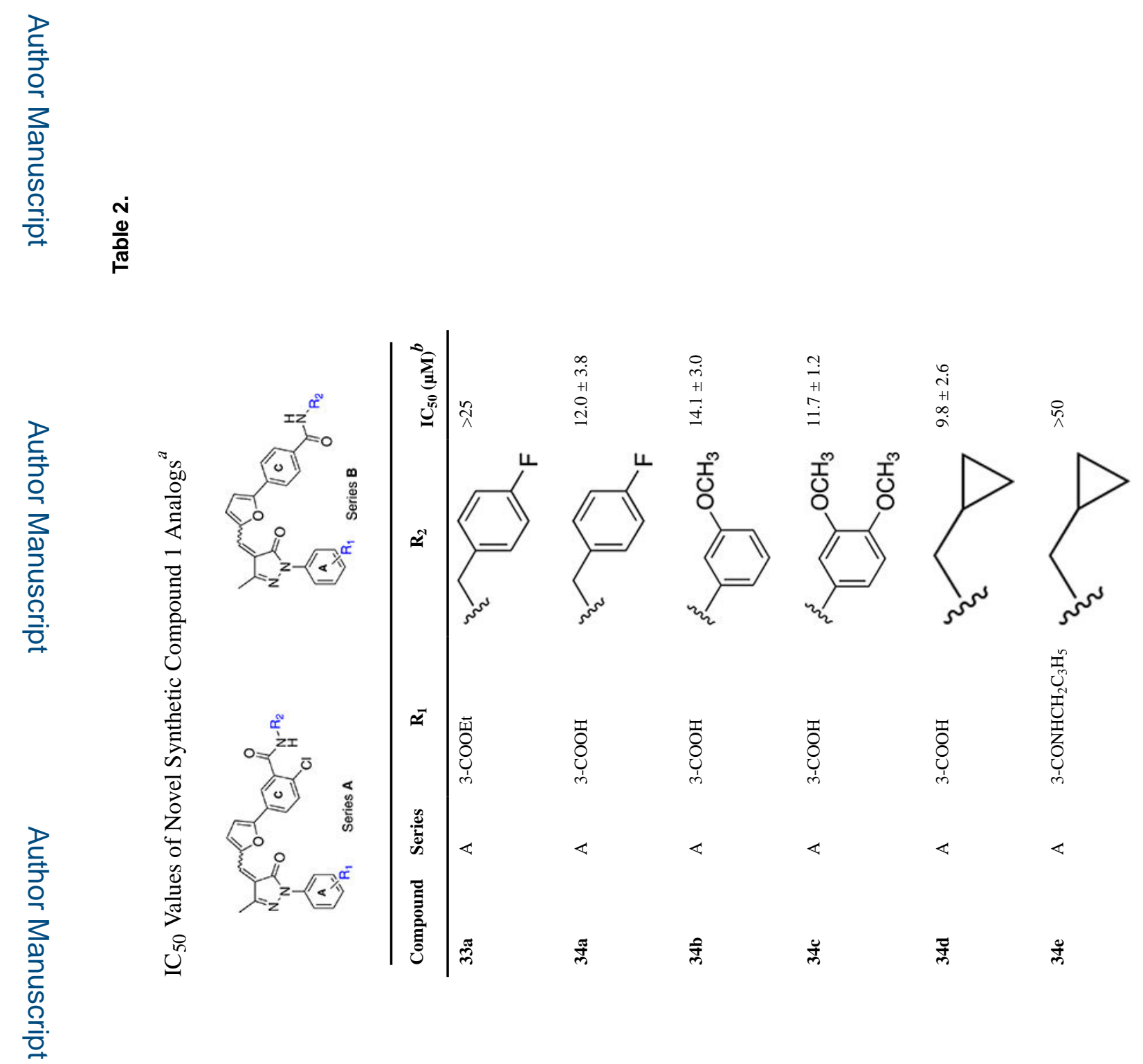


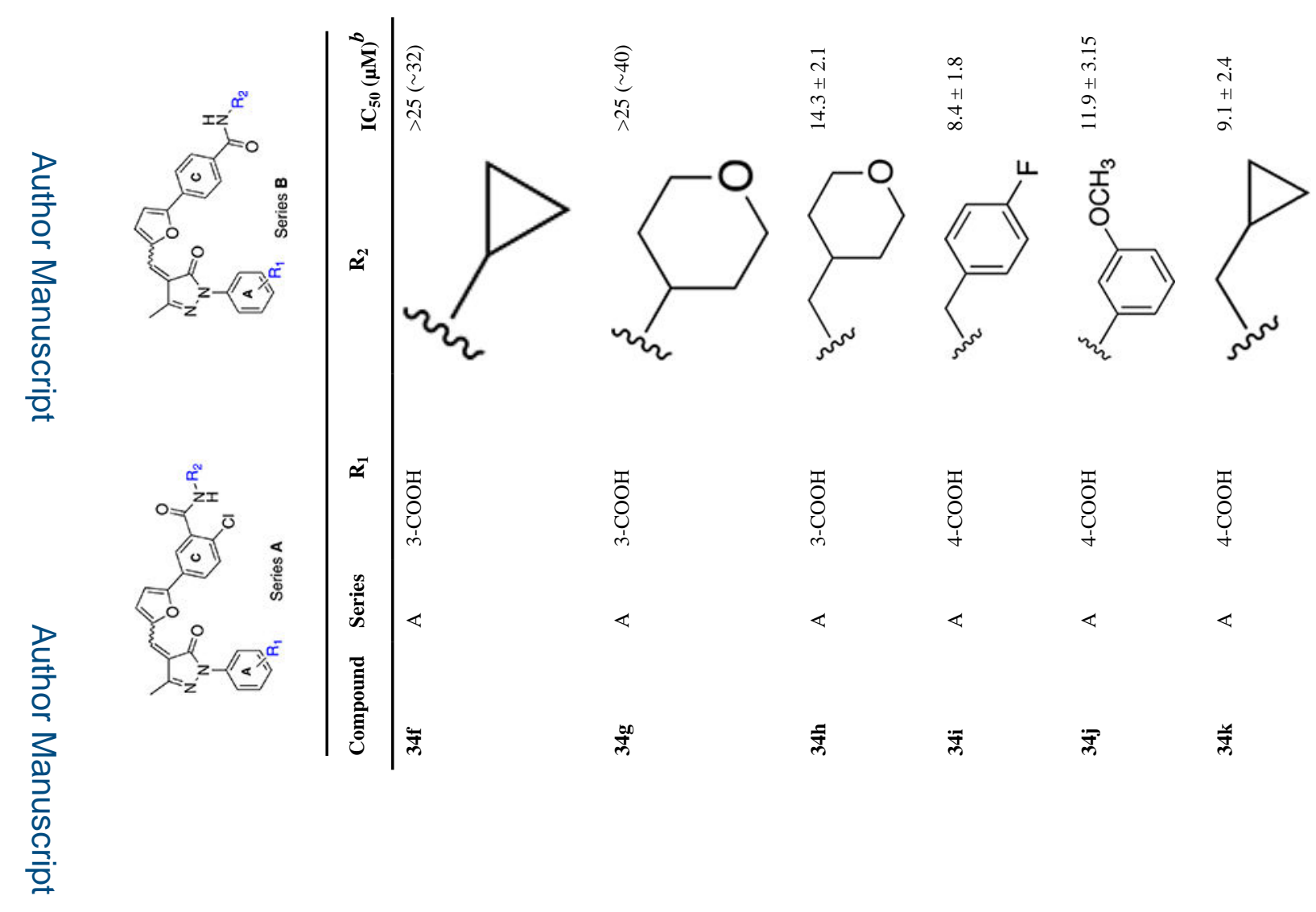

로을

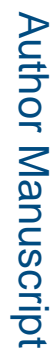

JMed Chem. Author manuscript; available in PMC 2020 July 07. 

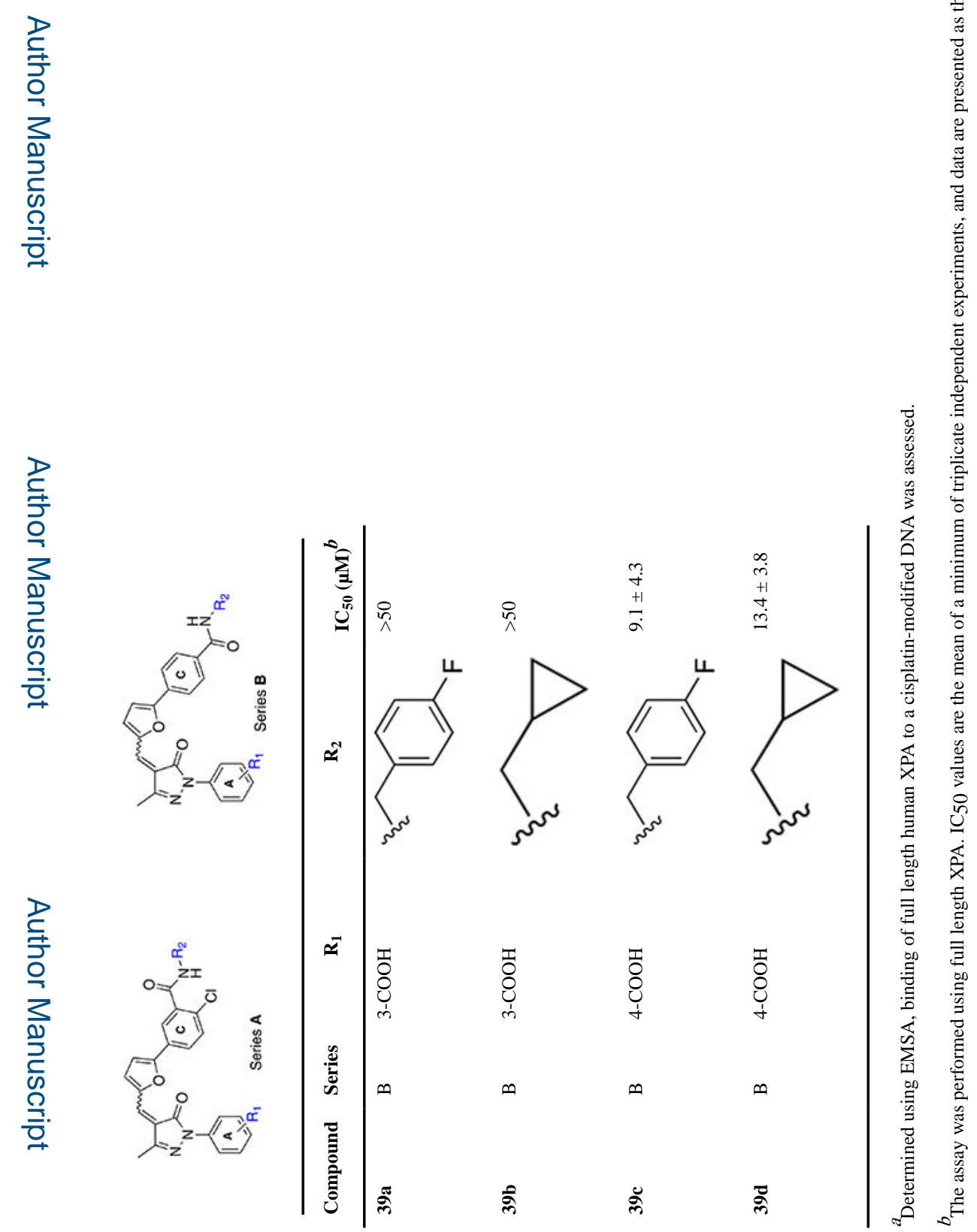

로을 